\title{
Metabolomics Study on Improvement of Mild Cognitive Impairment by SCPE: a UPLC-Q/TOF-MS Mass Spectrometric Study
}

\section{Zhitao Hou}

Heilongjiang University of Chinese Medicine

\section{Ailin Sun}

Heilongjiang University of Chinese Medicine

Qingshuang Wei

Beijing University of Chinese Medicine

\section{Changyuan He}

Heilongjiang University of Chinese Medicine

\section{Liying Song}

Heilongjiang University of Chinese Medicine

\section{Xianming Shao}

Heilongjiang University of Chinese Medicine

\section{Yang Li}

Heilongjiang University of Chinese Medicine

\section{Meng Wang}

Heilongjiang University of Chinese Medicine

\section{Tingting Mei}

Heilongjiang University of Chinese Medicine

\section{Yue Zhang}

Heilongjiang University of Chinese Medicine

\section{Hongcai Shang}

Beijing University of Chinese Medicine Affiliated Dongzhimen Hospital

\section{Zhongren Sun}

Heilongjiang University of Chinese Medicine

Jing Chen ( $\square$ chenjing6385@163.com )

Heilongjiang University of Chinese Medicine

\section{Research}

Keywords: Metabolomics, Mild Cognitive Impairment, UPLC-Q/TOF-MS mass spectrometry, SCPE, Traditional Chinese medicine 
Posted Date: September 8th, 2020

DOl: https://doi.org/10.21203/rs.3.rs-70833/v1

License: (c) (i) This work is licensed under a Creative Commons Attribution 4.0 International License. Read Full License 


\title{
Metabolomics Study on Improvement of Mild Cognitive Impairment by SCPE: a UPLC-Q/TOF-MS Mass Spectrometric Study
}

\author{
Zhitao Hou ${ }^{1,2 \dagger}$, Ailin Sun ${ }^{1 \dagger}$, Qingshuang Wei ${ }^{3 \dagger}$, Changyuan $\mathrm{He}^{1}$, Liying Song ${ }^{1}$, Xianming Shao ${ }^{1}$, Yang \\ $\mathrm{Li}^{1}$, Meng Wang ${ }^{1}$, Tingting Mei ${ }^{1}$, Yue Zhang ${ }^{1}$, Hongcai Shang ${ }^{2}$, Zhongren Sun ${ }^{1}$ and Jing Chen ${ }^{1 *}$
}

\footnotetext{
${ }^{*}$ Correspondence: chenjing2311@126.com

${ }^{1}$ College of Basic Medical and Sciences, Heilongjiang University of Chinese Medicine, 150040 Harbin, Heilongjiang, China Full list of author information is available at the end of the article

${ }^{\dagger}$ Equal contributor
}

\begin{abstract}
Background: Mild Cognitive Impairment $(\mathrm{MCl})$ is a common and easily neglected neurological disease in clinic. Aging is the main cause of the disease, but its exact pathogenesis is not yet clear. Sagacious Confucius' Pillow Elixir(SCPE) is a classic medicine for the treatment of amnesia in traditional Chinese medicine. The aim of this study was to prove the stability and reliability of SAMP8 mice as $\mathrm{MCl}$ model, and to find potential biomarkers for the transformation of mild cognitive impairment to dementia, so as to reveal the pathogenesis of mild cognitive impairment and the mechanism of SCPE at the whole animal level.
\end{abstract}

Methods: Senescence-accelerated P8 (SAMP8) mice were selected as the model of $\mathrm{MCl}$. Based on UHPLC/Q-TOF-MS platform, the effect of SCPE on $\mathrm{MCI}$ mice was evaluated by metabolomics to screen and identify brain tissue biomarkers and pathways of $\mathrm{MCl}$. T test of SPSS 22.0 software and meta-analysis software were used for univariate and multivariate analysis to screen and identify the biomarkers and pathways of brain tissue with mild cognitive impairment; SPSS 22.0 statistical software was used to conduct t-test on the total ionic strength corresponding to the different metabolites in each group, and to clarify the change mechanism of biomarkers and pathways in different doses of SCPE.

Results: From the point of view of brain metabolomics, $84 \mathrm{MCl}$-related biomarkers and 8 metabolic pathways were identified. It was found that different doses of SCPE could call back 36 same metabolic markers and 3 common metabolic pathways. And with the increase of dose, the number of SCPE callback metabolic markers gradually increased(LD:40; MD:48; HD:54), and the types of main metabolic pathways also changed to a certain extent (the biosynthesis of valine, leucine and isoleucine in low dose changed to cysteine and methionine metabolic pathway in middle and high dose), which may be related to the effect of aminoacyl tRNA.

Conclusions: The stability and reliability of $\mathrm{MCl}$ model, and the effectiveness of SCPE in the treatment of $\mathrm{MCl}$ were clarfied. Compared three different dose groups, the high dose SCPE group has more advantages.

\section{Keywords:}

Metabolomics; Mild Cognitive Impairment; UPLC-Q/TOF-MS mass spectrometry; SCPE; Traditional Chinese medicine 


\section{Background}

Mild Cognitive Impairment (MCI) refers to a state in which there are subjective and objective cognitive impairment, but the activities of daily living are basically normal [1]. MCI is a state of cognitive impairment between normal aging and Alzheimer's disease. It is an important early stage of $\mathrm{AD}[2]$. The specific pathogenesis of MCI is not clear. At present, some hypotheses and studies have put forward [3], including senile plaque caused by $\beta$-amyloid (A $\beta$ ), nerve fiber tangles caused by hyperphosphorylation of Tau protein, synaptic dysfunction, chronic inflammatory cascade reaction, oxidative stress damage, etc. are the main pathogenesis of MCI. Most of the drugs used in the treatment of MCI are the drugs used in the treatment of $\mathrm{AD}$, but the curative effect is still uncertain. However, traditional Chinese medicine prescription is a combination of Chinese herbal medicine, which can affect multiple targets and show synergistic therapeutic effect [4]. Therefore, it may be necessary to find a strategy to use traditional Chinese medicine to interfere with the progress of MCI.

SCPE is first published in Qianjin Yifang by Sun Simiao of Tang Dynasty. It is composed of tortoise shell (broiled), keel, acorus and thin-leaf milkwort root. The function of tortoise plastron in the prescription is to enrich yin and subdue yang, supplement essence and boost kidney, supplement the heart and nourish the blood, and has the function of supplementing water to restrict fire. The keel has the function of submerging yin fire and converging floating yang. Thin-leaf milkwort root, Acorus, dissolving phlegm to open the orifices, awakening the mind and boosting wisdom. The combination of the four drugs can restore interaction between the heart and the kidney, dissolving phlegm to open the orifices, awakening the mind and boosting wisdom, so as to achieve the effect of treating amnesia. We previously found that SCPE can improve cognitive function by reducing the content of $\mathrm{A} \beta$ protein in hippocampus and serum and the degree of phosphorylation of Tau protein $[5]$.

Metabolomics is another part of systems biology after genomics and proteomics. It mainly makes quantitative analysis of all metabolites in local tissues and body fluids of organisms, and looks for the relationship between metabolites and physiological and pathological changes in vivo [6]. The research objects of metabolomics are mostly small molecular substances with relative molecular weight less than 1000 Da. Compared with genomics and proteomics studies, metabolomics is more sensitive to biological changes, and small changes in gene and protein expression levels are magnified in metabolites, making the detection more real and accurate. At present, it is believed that metabolic group is the biological end point of all phenotypic groups and will play an important role in precision medicine. In this study, the mice model of MCI was studied by means of non-targeted metabolomics, in order to analyze the metabolic changes of brain tissue in mice with MCI and after SCPE treatment, to find the potential biomarkers of the transformation from MCI to dementia, and the possible mechanism of SCPE intervention in MCI. So as to reveal the pathogenesis of MCI and the mechanism of SCPE at the overall animal level. 


\section{Methods}

Drugs and chemical reagents

SCPE was purchased from Beijing Tongrentang Co,Ltd. (Beijing, China). 0.9\% saline was purchased from the first affiliated Hospital of Heilongjiang University of traditional Chinese Medicine (China). Formic acid (mass spectrum grade) was purchased from Thermo Fisher Scientific Company (USA). Ultra-pure water was purchased from Thermo Fisher Scientific (USA). Sodium formate was purchased from Thermo Fisher Scientific (USA). Pentobarbital was purchased from Sigma (USA) and methanol (mass spectrum grade) was purchased from Merck (USA). Ammonium acetate was purchased from Sigma Company (USA). Acetonitrile was purchased from Merck (USA).

\section{Animal and Model Construction}

Male SAMR1 mice, SAMP8 mice, with a body mass of 20-22g and 7 months old, were provided by the first affiliated Hospital of Tianjin University of traditional Chinese Medicine. (Tianjin, China). Before the formal study, all animals were fed adaptively for 7 days in a control room with a temperature of $20^{\circ} \mathrm{C}$ to $22^{\circ} \mathrm{C}$, a relative humidity of $40 \%$ to $60 \%$, and a light / dark cycle of 12 hours, and were free to eat sterile feed and autoclaved water.

All the animals were randomly divided into 5 groups with 6 animals in each group: blank group (SAMR1 mice, given the same volume of distilled water); MCI model group (SAMP8 mice, given the same volume of distilled water); SCPE low dose group (SAMP8 mice, SCPE solution $2.3 \mathrm{mg} / \mathrm{kg}$ ); Medium dose group (SCPE solution $4.6 \mathrm{mg} / \mathrm{kg}$ ); High dose group (SCPE solution $9.2 \mathrm{mg} / \mathrm{kg}$ ).

The experimental protocol was established, according to the ethical guidelines of the Helsinki Declaration and was approved by the Ethics Committee of Heilongjiang University of Traditional Chinese Medicine.

\section{Brain tissue sample processing}

Firstly, the brain tissue sample was thawed slowly at $4^{\circ} \mathrm{C}$ to completely thawed, then the methanol / acetonitrile / water solution precooled by $1 \mathrm{~mL}$ (at 2:2:1 volume ratio) was slowly added to the sample to fully swirl mixed concussion. After low temperature ultrasonic $30 \mathrm{~min}$, the supernatant was dried at $-20^{\circ} \mathrm{C}$ by $10 \mathrm{~min}, 14000 \mathrm{~g}$ centrifugation at $4^{\circ} \mathrm{C}$ for $20 \mathrm{~min}$. In mass spectrometry analysis, $100 \mu \mathrm{L}$ acetonitrile aqueous solution (acetonitrile:water $=1: 1$ ) was re-dissolved and swirled. The protein was precipitated by centrifugation at $14000 \mathrm{rpm} / \mathrm{min}, 4^{\circ} \mathrm{C}$ and centrifugation time was $15 \mathrm{~min}$. The supernatant was taken to the injection bottle for metabolomic analysis.

\section{UPLC-Q/TOF-MS analysis of brain tissue}

Chromatographic conditions: the brain tissue samples were separated by ACQUITY UPLC BEH C18 column (100mm to $2.1 \mathrm{~mm}, 1.7 \mu \mathrm{m}$, Waters, UK) with a column temperature of $50^{\circ} \mathrm{C}$ and a flow rate of $0.4 \mathrm{~mL} / \mathrm{min}$. The mobile phase is composed of $\mathrm{A}$ and $\mathrm{B}$ parts, $\mathrm{A}$ mobile phase: water $+0.1 \%$ formic acid; $\mathrm{B}$ mobile phase: methanol $+0.1 \%$ formic acid. The metabolites of brain tissue were eluted with the following gradients: $100 \% \mathrm{~A}$ for $0-2 \mathrm{~min}, 0-100 \% \mathrm{~B}$ for $2-11 \mathrm{~min}, 100 \% \mathrm{~B}$ for 
11-13min, 0-100\% A for 13-15min. The samples in the whole process are placed in an automatic injection container at $4^{\circ} \mathrm{C}$, and the sample volume of each sample is $10 \mu \mathrm{L}$. The random sequence was used to analyze the samples continuously. Insert QC samples into the sample sequence to evaluate and monitor the stability of the whole system and the reliability of the data.

Mass spectrometry conditions: the small molecular substances eluted from the chromatographic column were collected by high resolution tandem mass spectrometry Xevo G2-XS QTOF (Waters, UK). In the positive ion mode, the taper hole voltage and the tube voltage are $40.0 \mathrm{~V}$ and $3.0 \mathrm{kV}$, respectively. In anion mode, the taper hole voltage and capillary voltage are $40.0 \mathrm{~V}$ and $2.0 \mathrm{kV}$, respectively. Using MSE mode to collect CENTROID data, 50-1200 Da is the range of first-level scanning, and the scanning time is $0.2 \mathrm{~s}$. All parent ions are fragmented according to the energy of 20 to $40 \mathrm{eV}$, and the information of all fragments is collected, and the scanning time is also $0.2 \mathrm{~s}$. In the process of data acquisition, the real-time quality correction of LE signal is carried out every 3 seconds. At the same time, each interval of 10 samples to carry out a mixed quality control sample collection, has reached the evaluation of the stability of the instrument in the process of sample collection.

\section{Data preprocessing}

First of all, the original data obtained from the mass spectrometry were imported into the Progenesis QI software (version 2.2, QI) for peak extraction, in order to obtain the mass-charge ratio, ion area and retention time and other information related to the metabolites. The workflow of QI mainly includes: peak alignment, peak extraction and peak identification. Data preprocessing is completed using meta $\mathrm{X}$ software, including filling the missing values of the extracted data using the KNN method, removing low-quality ions (removing more than $50 \%$ of the missing ions in the QC samples, or removing more than $80 \%$ of the missing ions in the actual samples), and using QC-RSC (Quality control-based robust LOESS signal correction) method for correction.

\section{Principal component analysis}

In this part of the experiment, $\log 2$ conversion and proportion adjustment (scaling) are carried out on the data before PCA analysis. Pareto adjustment method (Pareto scaling) are used. PCA is mainly used to observe the trend of separation between groups in the experimental model, and whether there are outliers, and to reflect the degree of variation between groups and within groups from the original data.

\section{Partial least squares discriminant analysis}

In this part of the experiment, the $\log 2$ transformation is carried out before making the PLS-DA model, and the Pareto scaling method is used to adjust the scale of the data (scaling). Different from principal component analysis, PLS-DA is a supervised discriminant analysis statistical method, which can reflect the differences between classification groups to the greatest extent. This method uses partial least square regression to establish the relationship model between metabolite expression and sample categories to realize the modeling and prediction of sample categories. 
Identification of differential metabolites in brain tissue

Firstly, according to the difference variables obtained from the analysis, the compounds in the brain tissue were identified by matching with the databases such as HMDB, METLIN, KEGG, etc., and the output included the data matrix including the name of the metabolites, HMDB ID, mUniz, RT, the ion form of adducts and so on.

\section{Analysis of metabolic pathway in brain tissue}

The identified brain tissue differential metabolites were introduced into MetaboAnalyst (http://www.metaboanalyst.ca) database in the format of HMDB ID for metabolic pathway analysis. The whole process mainly includes data processing, standardization, statistical analysis and advanced functional interpretation, in order to reveal the possible biological mechanisms.

\section{Results}

The model is established according to the grouping. And the reliability of the model is verified through the analysis. The differential metabolites are then determined. Then the metabolites of different dose groups are analyzed to study the effect of SCPE on MCI.

The establishment of models in each group

\section{Principal component analysis}

In order to more intuitively reflect the clustering between groups and within groups, the ion strength information of each sample in each group is imported into SIMCAP 14.1 software, and the specific results of principal component analysis (PCA) in three-dimensional mode are shown in Figure 1.

As can be seen from Figure 1, the intra-group clustering of blank group (Cont), model group (Mod), low (LD) medium (MD) and high (HD) dose group is good, and the clustering between groups has a tendency of separation to some extent, especially the (Cont) of blank group is obviously separated from that of model group. Although each dose of SCPE did not completely coincide with the intragroup clustering of the blank group, it also showed a trend towards the blank group, in which the tendency under the positive ion mode (pos) was more obvious, indicating that SCPE has the callback function of MCI biomarkers.

\section{PLS-DA analysis}

In this part, metaX software was used to establish the PLS-DA model among blank group (Cont), model group (Mod), low (LD) medium (MD) and high (HD) dose groups. It was found that the parameters (R2 and Q2) were higher, indicating that the current PLS-DA model was more reliable. At the same time, the parameters R2 and Q2 of the model were tested 200 times, and the PLS-DA models of each group in positive and negative ion mode were obtained (Figure 2).

As can be seen from Figure 2, the left side is the PLS-DA score map of each comparison group in the positive ion mode, and the right side is the PLS-DA score map of each comparison group in the negative ion mode, and the Abscissa represents the first principal component $\mathrm{PC} 1$, ordinate represents the second principal 
component PC2. Each point in the graph represents a sample, and the discreteness of the two color symbols represents the distribution trend of the two groups of samples on the PC1 and PC2 axes, respectively. The values of $\mathrm{Q} 2$ and $\mathrm{R} 2$ in the positive and negative ion mode were higher than 0.5 and almost close to 1 , and the trend of each dose group was similar to that of the blank group, that is, it was obviously separated from the model group, indicating that SCPE has a better positive intervention effect.

\section{Screening and identification of differential metabolites}

The ions detected by mass spectrometry conditions were analyzed by SPSS22.0 software $\mathrm{T}$ test and metaX analysis software for univariate and multivariate analysis, so as to screen the differential metabolites between groups.

\section{Univariate analysis}

In this part, T-test and multiple of variation analysis (Fold change analysis, FC analysis) were used to make statistics, and the p-value generated by statistical test was corrected by FDR to get Qmurvalue. the final result showed two indexes of difference multiple (Fold Change, FC) and q-value in the form of volcanic map (Volcano plot). The condition for screening differential metabolites was (FC) $\geq 1.2$ or $\leq 0.8333$, Q value ; 0.05 . the results are shown in Table 1 and Figure 3.

Table 1 contains the differential ions between the two groups, and shows the general situation of the down-regulation of the differential ions of the model group (Mod) relative to the blank group (Cont) (for example, the group is ContvsMod, indicating that the Cont group is relative to the Mod group), as well as the identification of the first and second levels.

Log2 (fold change) is Abscissa, and the negative logarithm of q-value-log10 (qvalue) is ordinate. The dots with fold change $\leq 0.8333$ or $\geq 1.2$, q-value; 0.05 were marked red, and the other dots were purple. The red dots were the differential ions screened by univariate analysis.

\section{Multivariate analysis}

Because simple univariate analysis can not dig out reliable metabolic markers. Therefore, on the basis of univariate analysis, we used multivariate statistical analysis of the VIP values of the first two principal components of the PLS-DA model, combined with univariate analysis of the multiple of difference (fold-change) and q-value values in the volcano map to screen differentially expressed metabolites. That is, the screening conditions of this part are as follows: 1) VIP $\geq 1$; 2) fold change $\geq 1.2$ or $\leq 0.8333 ; 3)$ q-value ; 0.05 , the three intersect, and the common ions, namely differential ions, are identified by ProgenesisQI (version2.2) software. The differential ions and the identification results are shown in Table 2 and Figure 4 .

The preliminary screened differential ions are analyzed by cluster analysis, which is presented in the form of VIP diagram and heat map, as shown in Figure 4.

Each row in the picture represents a differential ion, each column represents a sample, and different colors represent different intensities, ranging from green to red, indicating that the intensity is from low to high. 
Combined with Table 2 and Figure 4, after multivariate statistical analysis (VIP $\geq$ 1), the range of differential metabolites was further reduced, in which the differential ions in positive ion mode were screened from 313 to 302 and the differential ions in negative ion mode were screened from 356 to 169 in univariate statistical analysis. It provides a more clear candidate differential ion set for further identification of differential metabolites.

\section{Identification of differential metabolites}

Through the above differential metabolite screening process, 471 differential ions were obtained, including 302 in positive ion mode and 169 in negative ion mode. Compared with the blank group(Cont), the differential ions in the model group(Mod) showed obvious up-down relationship. To further identify potential metabolic markers of MCI, we combined the matching degree in mass spectrometry and used related mass spectrometry websites such as: Human Metabolome Database $^{[1]}$; Lipidomics Gateway ${ }^{[2]}$; METLIN Metabolite Database ${ }^{[3]}$. Differential metabolites were identified by $\mathrm{SDBS}^{[4]}$ and related literature reports. Results A total of 84 biomarkers were identified, including 45 in positive ion mode (32 upregulated and 13 down-regulated). It mainly includes angiotensin, leukotriene D4, cetane diacid, (3z,6z) 3-nonadienal, isoprenaline, D-ornithine, methoxamine, citrulline, coriander aldehyde, isopropylmalic acid, etc. Thirty-nine (31 up-regulated and 8 down-regulated) in negative ion mode, including L-rhamnose, N-acetyl-Lhistidine, coriander glycoside, arginine, ornithine, 2-isopropylmalic acid, L-leucine, $\gamma$-glutamyl-selenomethylselenocysteine, xylobiose, L-positive leucine, etc. the specific results are shown in Table 3, the content information is shown in Figure 5 and 6 , and the cluster analysis is shown in Figure 7 and 8.

The specific contents of 84 differential metabolic markers identified were analyzed, that is, the total ionic strength of mass spectrometry and differential metabolites in each group was tested by SPSS22.0 software, and the results were expressed in the form of mean \pm standard deviation (mean $\pm \mathrm{SD}$ ). If the metabolite screened in the previous step is not statistically significant between the model group and the blank group ( $\mathrm{P} i 0.05)$, it means that the metabolite has no significant correlation with $\mathrm{MCI}$, but when the comparison between the two groups is $\mathrm{P} ; 0.05$, it is considered to be statistically significant, and the metabolite is the metabolic marker of MCI (Figure 5 and 6).

From Figure 5 and 6 , it can be seen that the 84 metabolic markers in the model group changed significantly compared with the blank group, and the difference was statistically significant (P-value ; 0.01). It is suggested that the stability of MCI model and the reliability of metabolite screening. In order to better reflect the changes of differential metabolites, we carried out cluster heat map analysis of 84 metabolic markers. Each row in Figure 7 and 8 represents the ions corresponding to a biomarker, and each column represents a sample. Different colors represent different intensity, from blue to red, indicating that the intensity is from low to high.

\footnotetext{
${ }^{[1]}$ http://www.hmdb.ca/

${ }^{[2]}$ http://www.lipidmaps.org/

${ }^{[3]} \mathrm{http}: / /$ metlin.scripps.edu/

${ }^{[4]}$ http://riodb01.ibase.aist.go.jp/sdbs/cgi-bin/direct_frame_top.cgiSp
} 


\section{Biological interpretation of metabolic markers}

After the identification and verification of metabolic markers, we found a total of 84 metabolic markers, which were sorted according to FC and VIP values. In the positive ion mode (pos), the top 10 metabolites are angiotensin, leukotriene D4, cetane dicarboxylic acid, (3z,6z) 3,6-nonadienal, isoprenaline, D-ornithine, methoxyamine, citrulline, coriander aldehyde, isopropylmalic acid, etc; In the anion mode(neg), the top 10 metabolites are: L-rhamnose, N-acetyl-L-histidine, coriander glycoside, arginine, ornithine, 2 -isopropylmalic acid, L-leucine, $\gamma$-glutamylselenomethylselenocysteine, xylobiose, L-n-leucine and so on.

Angiotensin is a peptide with strong vasoconstrictive effect, which is mainly involved in the regulation of human blood pressure[7]. Leukotriene D4[8] is an important inflammatory mediator in human body, which participates in the process of chronic inflammation and oxidative stress injury in the brain. Hexadecanedioic acid[9] is an intermediate substance involved in fatty acid metabolism. (3z,6z) 3,r6nonadienal is a metabolic intermediate with aromatic odor. At present, there is mainly research in the field of botany, but there is little research in the field of animal and medicine[10]. Isoproterenol[11] is a common $\beta$-adrenoceptor agonist, which mainly promotes myocardial contraction, increases cardiac output, dilates blood vessels and accelerates heart rate. D-ornithine, arginine and ornithine are all intermediates in the metabolism of urea, which are mainly involved in the production of urea in human body[12]. The main function of methoxamine is to stabilize the arterial pressure of the body, which can effectively prevent low perfusion of brain tissue caused by the decrease of blood pressure[13]. Citrulline is an $\alpha$-amino acid produced from ornithine in the urea cycle, which is mainly involved in the chronic inflammatory response of the body[14]. Pine and cypress aldehyde has anti-inflammatory, sedative, bacteriostatic and analgesic effects[15]. L-rhamnus gum sugar has good intestinal permeability and has a strong correlation with the metabolism of bacteria, viruses and other microorganisms [16]. N-acetyl-L-histidine can inhibit neuronal apoptosis and HIV virus replication[17]. Cilantro is a neuroprotective agent, which mainly inhibits neuroinflammation and improves the formation of neuronal edema in brain tissue. The substance has the advantages of clear chemical structure, stable content and clear metabolic mechanism in the body[18]. $\gamma$-glutamylselenomethylselenocysteine is a selenium-rich metabolite, which is mainly studied in the field of fortified food nutrition[19]. Xylobiose can enhance the proliferation of intestinal bifidobacteria and improve gastrointestinal function [20]. L-leucine and L-leucine are the main products of amino acid metabolism and participate in the pathogenesis of neuroinflammation and mental disorders[21].

\section{Pathway Analysis of Biological Metabolic markers}

In order to more intuitively reflect the potential mechanism of 84 biomarkers identified, this part analyzed their pathways, mainly based on KEGG and HMDB database to annotate metabolite metabolic pathways. Using MetaboAnalyst ${ }^{[5]}$ online software analysis tool, the differential metabolites identified in the previous step were used for pathway analysis. The input format of metabolites was Compoundname/HMDBID/KEGGID, and Impact was set to greater than 0.1 as the screening

${ }^{[5]}$ https://www.metaboanalyst.ca/ 
criteria for potential main metabolic pathways. After analysis, 8 main metabolic pathways related to MCI were obtained, which were as follows: D-Arginine and D-ornithine metabolism; Alanine, aspartate and glutamate metabolism; Phenylalanine metabolism; Arginine and proline metabolism; Valine, leucine and isoleucine biosynthesis; Cysteine and methionine metabolism; Aminoacyl-tRNA biosynthesis; D-Glutamine and D-glutamate metabolism.

Figure 9 shows the metabolic pathway analysis of MCI biomarkers. Y axis represents $\mathrm{p}$ value (from path enrichment analysis), $\mathrm{X}$ axis represents pathway impact value (from path topology analysis). The color of each node is made based on the $\mathrm{p}$ value, from light to deep represents the $\mathrm{p}$ value from large to small, and the radius of the node represents the impact value from small to large based on its corresponding pathway impact value. This part marks the first 15 metabolic pathways in the diagram, and the sequence number and the content represented in the diagram are consistent with the information in Table 4. Among them, 1-8 is the potential metabolic pathway obtained after screening.

84 major metabolic markers related to mild cognitive impairment were identified after further analysis of metabolic markers and 8 major metabolic pathways were obtained by MetaboAnalyst and Enrichment analysis. The relevant data were imported into Cytoscape3.7.1 software to visualize the results.

As can be seen from Figures 11 and Table 4, the metabolites involved in DArginine and D-ornithine metabolism are: D-ornithine and ornithine, Account for $2 / 8$ of the total; The metabolites involved in Alanine,aspartate and glutamate metabolism are: N-Acetyl-L-aspartic acid, L-Aspartic acid, L-Asparagine, LGlutamic acid, Account for 4/24 of the total; The metabolites involved in Phenylalanine metabolism are: L-Phenylalanine, D-Phenylalanine, 2-Phenylacetamide, transCinnamic acid, 4-Hydroxycinnamic acid, Enol-phenylpyruvate, Phenylacetaldehyde, Account for 7/45 of the total; The metabolites involved in Arginine and proline metabolism are: L-Glutamic acid, L-Aspartic acid, Citrulline, Ornithine, Account for $4 / 77$ of the total; The metabolites involved inValine, leucine and isoleucine biosynthesis metabolism are: 2-Isopropylmalic acid, L-Valine, Isopropylmaleate, LLeucine, L-Isoleucine, Account for 5/27 of the total; The metabolites involved in Cysteine and methionine metabolism are: L-Aspartic acid, L-Cystathionine, 2Aminoacrylic acid, Account for 3/56 of the total; The metabolites involved in Aminoacyl-tRNA biosynthesis are: L-Asparagine, L-Phenylalanine, L-Valine, LIsoleucine, L-Threonine, L-Aspartic acid, L-Glutamic acid, Account for 7/75 of the total; The metabolites involved in D-Glutamine and D-glutamate metabolism are: L-Glutamic acid, Account for 1/11 of the total.

\section{Regulatory effect of SCPE on metabolic markers of $\mathrm{MCl}$}

In order to clarify the regulatory mechanism of SCPE on 84 related biomarkers identified in the previous step, we used SPSS22.0 statistical software to test the total ionic strength of different metabolites in each group, and the results were expressed in the form of mean \pm standard deviation (mean $\pm \mathrm{SD}$ ). Compared with the model group, the $\mathrm{P}_{i} 0.05$ of each dose group was considered to be statistically significant, and $\mathrm{P} ; 0.01$ represented a significant difference. It is suggested that different doses of SCPE can regulate the biomarker. 
Screening and pathway analysis of biomarkers of low dose SCPE callback MCI

Through T-test and consulting the basic information of each metabolite in the literature, it is concluded that low dose SCPE can call back 40 biomarkers of MCI. In this part, the 40 biomarkers screened are shown in Table 5, metabolite intensity histogram (Figure 12-A) and heat map (Figure 12-B), which can directly reflect the changes of effective biomarkers of low dose SCPE intervention in MCI.

In order to clarify the action mechanism of the 40 biomarkers obtained in the previous step, this part analyzed their pathways, mainly based on KEGG and HMDB database to annotate metabolite metabolic pathways. Using MetaboAnalyst $^{[6]}$ online software analysis tool, the pathway analysis, metabolite input format of the differential metabolites identified in the previous step is Compoundname/HMDBID/KEGG ID, and the Impact value is set to greater than 0.1 as the screening criteria for potential main metabolic pathways. After analysis, four main metabolic pathways were obtained, which were as follows: Phenylalanine metabolism; Alanine, aspartate and glutamate metabolism; Valine, leucine and isoleucine biosynthesis; D-Glutamine and D-glutamate metabolism.

Taking Impact as a factor, the enrichment analysis of each metabolic pathway of low dose SCPE intervention MCI is shown in figure 14, in which light red represents the main metabolic pathway and blue is the related metabolic pathway.

After further analysis of 40 biomarkers of low dose SCPE callback MCI, it was found that 11 metabolites were significantly call back (P-value ; 0.01): Hexadecanedioic acid, Eicosadienoic acid, L-Isoleucine, Leukotriene D4, 11Z-Eicosenoic acid, D-Phenylalanine, Isopropylmaleate, trans-Cinnamic acid, N1-Acetylspermine, L-Phenylalanine, Gibberellin A15 open lactone. Four main metabolic pathways and their participating metabolites were obtained by MetaboAnalyst and Enrichmentanalysis analysis: (1) Phenylalanine metabolism: L-Phenylalanine, D-Phenylalanine, trans-Cinnamic acid, Account for 3/45 of the total; (2) Alanine, aspartate and glutamate metabolism: L-Glutamic acid, Account for 1/24 of the total; (3) Valine, leucine and isoleucine biosynthesis: 2-Isopropylmalic acid, Isopropylmaleate, L-Leucine, L-Isoleucine, Account for $4 / 27$ of the total; (4) D-Glutamine and Dglutamate metabolism: L-Glutamic acid, Account for $1 / 11$ of the total.

Screening and pathway analysis of biomarkers of medium dose SCPE callback MCI After T-test and consulting the basic information of each metabolite in the literature, it was determined that the medium dose SCPE could call back 48 biomarkers of MCI. The 48 biomarkers screened were shown in Table 7, metabolite intensity histogram (Figure 15-A) and heat map (Figure 15-B), which could directly reflect the changes of effective biomarkers of MCI intervened by medium dose SCPE.

The pathways of 48 metabolites from the medium dose SCPE callback obtained in the previous step were analyzed, and the metabolic pathways were annotated mainly based on KEGG and HMDB database. Using MetaboAnalyst ${ }^{[7]}$ online software analysis tool, the input format of metabolites was Compoundname/HMDBID/KEGG ID, and Impact was set to greater than 0.1 as the screening criteria for potential

[6] https://www.metaboanalyst.ca/

${ }^{[7]}$ https://www.metaboanalyst.ca/ 
main metabolic pathways. After analysis, four main metabolic pathways were obtained (Figure 16 and Table 8): Phenylalanine metabolism; Alanine, aspartate and glutamate metabolism; Cysteine and methionine metabolism; D-Glutamine and Dglutamate metabolism.

Taking Impact as a factor, the enrichment analysis of each metabolic pathway of medium dose SCPE intervention MCI is shown in figure 17, in which red represents the main metabolic pathway and blue is the related metabolic pathway.

After further analysis of 48 biomarkers of medium dose SCPE callback MCI, it was found that there were 17 significant call back metabolites (P-value ; 0 01), of which 9 metabolites were the same as those of low dose callback, namely: Hexadecanedioic acid, Eicosadienoic acid, L-Isoleucine, Leukotriene D4, 11Z-Eicosenoic acid, trans-Cinnamic acid, N1-Acetylspermine, L-Phenylalanine, Gibberellin A15 open lactone. The 8 different significant callback metabolic markers are: L-Carnitine, 4-Hydroxyifosfamide, Angiotensin, 16-Hydroxy hexadecanoic acid, Ricinoleic acid, 5-Hydroxyindoleacetate, Methdilazine, L-Leucine. Four main metabolic pathways and their participating metabolites were obtained by MetaboAnalyst and Enrichment analysis: (1) Phenylalanine metabolism: L-Phenylalanine, D-Phenylalanine, trans-Cinnamic acid, 4-Hydroxycinnamic acid, Account for 4/45 of the total; (2) Alanine, aspartate and glutamate metabolism: L-Glutamic acid, Account for 1/24 of the total; (3) Cysteine and methionine metabolism: L-Cystathionine, Account for 1/56 of the total; (4) D-Glutamine and D-glutamate metabolism: L-Glutamic acid, Account for $1 / 11$.

Screening and pathway analysis of biomarkers of high dose SCPE callback MCI After T-test and consulting the known basic information of each metabolite in the literature, it was determined that high-dose SCPE could call back 54 biomarkers of MCI. The 54 biomarkers screened were shown in Table 9, metabolite intensity histogram (Figure 18-A) and heat map (Figure 18-B), which could directly reflect the changes of effective biomarkers of high dose SCPE intervention in MCI.

The pathways of 54 metabolites from the high dose SCPE callback obtained in the previous step were analyzed. Firstly, the metabolic pathways were annotated based on KEGG and HMDB database, and then the MetaboAnalyst ${ }^{[8]}$ online software analysis tool was used to set the input format of metabolites to Compoundname/HMDBID/KEGG ID, and set Impact greater than 0.1 as the screening criteria for the main metabolic pathways. After analysis, four main metabolic pathways were obtained (Figure 19 and Table 10): Phenylalanine metabolism; Alanine, aspartate and glutamate metabolism; Cysteine and methionine metabolism; D-Glutamine and D-glutamate metabolism.

Taking Impact as a factor, the enrichment analysis of each metabolic pathway of high dose SCPE in MCI is shown in figure 20, in which crimson represents the main candidate metabolic pathway and blue is the related metabolic pathway.

After further analysis of 54 biomarkers of high-dose SCPE callback MCI, it was found that there were 23 significant callback metabolites (P-value i 0.01), including 9 metabolic markers of low dose significant callback: Hexadecanedioic acid, L-Isoleucine, Leukotriene D4, 11Z-Eicosenoic acid, Isopropylmaleate, trans-Cinnamic acid, N1-Acetylspermine, L-Phenylalanine, Gibberellin

${ }^{[8]}$ https://www.metaboanalyst.ca/ 
A15 open lactone; Metabolites with significant pullback in medium dose: LCarnitine, 4-Hydroxyifosfamide, Methdilazine; Metabolites of their own significant callbacks: Piperidine, 1-Phenyl-1, 2-propanedione, o-Cresol, LysoSM(d18:1), (3Z,6Z)-3,6-Nonadienal, Alpha-Tocotrienol, Mannitol 1-phosphate, beta-Tyrosine, L-Norleucine, 2-Isopropylmalic acid, (S)-2-(Hydroxymethyl)glutarate. Four main metabolic pathways and their participating metabolites were obtained by MetaboAnalyst and Enrichment analysis analysis: (1) Phenylalanine metabolism: LPhenylalanine, D-Phenylalanine, trans-Cinnamic acid, 4-Hydroxycinnamic acid, Account for 4/45 of the total; (2) Alanine, aspartate and glutamate metabolism: L-Glutamic acid, Account for 1/24 of the total; (3) Cysteine and methionine metabolism: L-Cystathionine, 2-Aminoacrylic acid, Account for 2/56 of the total; (4) D-Glutamine and D-glutamate metabolism: L-Glutamic acid, Account for $1 / 11$ of the total.

\section{Discussion}

Venn analysis of metabolic markers

The metabolic markers of the model and the callback of low, medium and high dose SCPE were intersected by Venn map to obtain the common metabolic markers and unique metabolic markers of different doses of SCPE in MCI. The effects of SCPE on brain metabolic markers with doses were analyzed, the possible mechanism of different doses was revealed and the best dose of MCI was determined (Figure 21).

As can be seen from figure 21, LD, MD and HD can call back 36 common metabolic markers; LD and MD can call back 39 common metabolic markers, including 3 unique metabolic markers; LD and HD can call back 37 common metabolic markers, including one unique metabolic marker; $\mathrm{MD}$ and $\mathrm{HD}$ can call back 45 common metabolic markers, including 9 unique metabolic markers. In addition, HD can call back 8 unique metabolic markers. The basic information of callback metabolic markers in each dose group is shown in Table 11.

As can be seen from figure 21 and Table 11, the metabolic markers of MCI in each dose group of SCPE account for $68.9 \%$ of the total, indicating that SCPE, a classic prescription for the treatment of amnesia in traditional Chinese medicine, has a better effect on improving cognitive function. Among them, the metabolic markers of MCI47.7 can be recalled in the low dose group, the metabolic markers of MCI57.2\% can be recalled in the middle dose group, and $64.3 \%$ of the metabolic markers can be recalled in the high dose group, while there are a small number of unique metabolic markers in different doses of SCPE. The results showed that with the change of dose, the types of MCI metabolic markers changed to a certain extent, and the effect of high dose was the most obvious. The analysis of the reason may be related to the effect of drug concentration on the upstream and downstream relationship of metabolites in the metabolic pathway.

\section{Analysis of metabolic pathway}

The common and unique metabolic markers of the three groups were enriched and analyzed to make clear the action pathway of the common metabolic markers of each dose of SCPE callback MCI and the significance of the specific metabolic markers under different doses. The metabolic mechanism of different doses was analyzed to explore the relationship between metabolic pathways. 
As can be seen from Figure 22-A, compared with the model group, the metabolic markers of each dose group were significantly adjusted. It is suggested that common metabolic markers play an important role in the process of SCPE intervention in MCI.

As can be seen from Figure 22-B, red represents positive correlation and blue represents negative correlation. Comprehensive analysis showed that there was a strong positive correlation between Hexadecanedioic acid and (S)-2(Hydroxymethyl)glutarate, Angiotensin, 5-Hydroxyindoleacetate, L-Glutamic acid, D-Phenylalanine, Toluene, L-Leucine, Isoproterenol, Leukotriene D4, Methdilazine, o-Cresol, trans-Cinnamic acid, L-Isoleucine, L-Phenylalanine (R \& 0.8); There was a strong negative correlation between 16-Hydroxy hexadecanoic acid and 11ZEicosenoic acid, o-Cresol, Coniferyl aldehyde, L-Rhamnulose, N-Acetyl-L-histidine, D-Phenylalanine, trans-Cinnamic acid, Toluene, Hexadecanedioic acid, L-Isoleucine, L-Phenylalanine $\left(\mathrm{R}_{\mathrm{j}}-0.80\right)$. It is suggested that cetyldicarboxylic acid and 16hydroxyhexadecanoic acid play an important role in the pathogenesis of MCI and the improvement of cognitive impairment by SCPE. It is found that[22], hexadecanoic acid and 16-hydroxyhexadecanoic acid are important substances involved in fatty acid metabolism. However, abnormal fatty acid metabolism is gradually regarded as an important factor leading to cognitive impairment, but the research on fatty acid spectrum of cognitive disorders is not clear at present[23]. This part of the study provides two important metabolic markers to reveal the pathogenesis of MCI and the improvement of SCPE from the perspective of fatty acid metabolism.

As can be seen from Figure 22-C, three candidate pathways with Impact greater than 0.1 were obtained by MetPA analysis of 36 metabolic markers, which are: Phenylalanine metabolism; Alanine, aspartate and glutamate metabolism; DGlutamine and D-glutamate metabolism.

As can be seen from Figure 22-D, 4 candidate pathways with Impact greater than 0.1 were obtained by MetPA analysis of 40 metabolic markers of LD callback: Phenylalanine metabolism; Alanine, aspartate and glutamate metabolism; Valine, leucine and isoleucine biosynthesis; D-Glutamine and D-glutamate metabolism.

As can be seen from Figure 22-E, 4 candidate pathways with Impact greater than 0.1 were obtained by MetPA analysis of 48 metabolic markers of MD callback: Phenylalanine metabolism; Alanine, aspartate and glutamate metabolism; Cysteine and methionine metabolism; D-Glutamine and D-glutamate metabolism.

As can be seen from Figure 22-F, 4 candidate pathways with Impact greater than 0.1 were obtained by MetPA analysis of 54 metabolic markers of HD callback: Phenylalanine metabolism; Alanine, aspartate and glutamate metabolism; Cysteine and methionine metabolism; D-Glutamine and D-glutamate metabolism.

It can be seen from Figure $22-\mathrm{C} \sim \mathrm{F}$ that 3 of the four main metabolic pathways did not change significantly with the increase of dose, suggesting that phenylalanine, alanine, aspartic acid and glutamate did not change significantly, and the main metabolites of D-glutamine and D-glutamate did not change significantly, indicating that the above three metabolic pathways are the main metabolic pathways of MCI and SCPE intervention in MCI.

Phenylalanine metabolism is a metabolic pathway closely related to cognitive impairment. Phenylalanine is an essential amino acid in the human body, which 
metabolizes to tyrosine in the liver tissue, and then participates in the synthesis of brain neurotransmitters such as dopamine(DA), 5-hydroxytryptamine (5-HT) and norepinephrine (NE) [24]. A variety of factors can lead to abnormal metabolism of phenylalanine, including cerebrovascular diseases, neuroimmune diseases, genetic diseases and other factors, leading to the disturbance of tyrosine production and the increase of phenylalanine / tyrosine ratio in blood[25]. Studies have found that high levels of phenylalanine or phenylalanine / tyrosine will destroy the activity of Na+-K+-ATP on the synaptic serosa, resulting in cognitive impairment, mental retardation, depression and other diseases[26].

Alanine, aspartic acid and glutamic acid are non-essential amino acids in human body, and this metabolic pathway is mainly involved in aerobic glycolysis and tricarboxylic acid cycle[27]. Among them, alanine metabolism is closely related to sugar metabolism. Glycolysis provides pyruvate for the biosynthesis of alanine, which is then formed by combined deamination or transamination. In addition, as another source of non-essential amino acids, alanine is the product of tryptophan metabolism. Aspartic acid is the precursor of $\alpha$-ketoglutaric acid, and glutamic acid is the precursor of oxaloacetic acid, which is mainly involved in aerobic glycolysis and tricarboxylic acid cycle, and is closely related to the uptake of free glucose by brain tissue to maintain normal brain metabolism[28, 29, 30].

The metabolism of D-glutamine and D-glutamate is closely related to aging, and the metabolic level of D-glutamine and D-glutamate will be disordered in the aging body[31]. Among them, D-glutamate is an important neurotransmitter in the nervous system, which is mainly involved in the regulation of human memory, emotion and intelligence. However, the study found that[32], high levels of glutamate can induce neurotoxicity, brain tissue degeneration, intellectual and emotional disorders and so on. Glutamine can form glutamic acid with perdeamination.

As can be seen from Figure 22 and 23, the metabolic pathway with Impact greater than 0.1 changed from the biosynthesis of valine, leucine and isoleucine in low dose to the metabolic pathway of cysteine and methionine in middle and high dose. The reason may be related to the biosynthesis of aminoacyl tRNA enriched by the common metabolites.

Aminoacyl refers to the univalent group left after the hydroxyl group of amino acid molecule is removed. tRNA mainly performs the translation function from DNA to protein. The process of tRNA carrying certain amino acids to ribosomes needs to be completed by a variety of aminoacyl-tRNA synthetases[33, 34, 35]. Aminoacyl-tRNA synthesis mainly affects the synthesis of a variety of proteins, thus affecting the secretion of neurotransmitters in brain tissue, the synthesis of related metabolic enzymes, the synthesis of iconic proteins and neuronal regeneration. It has been found that the metabolism of valine, leucine and isoleucine is closely related to aminoacyl tRNA, which further affects the biological metabolism of related amino acids and proteins in human body. at present, this metabolic pathway has been proved to be related to the pathogenesis of hypertension and coronary heart disease[36, 37, 38]. Aminoacyl-tRNA is also an important participant in the metabolism of cysteine and methionine, which mainly affects the production of blood homocysteine. It has been found that homocysteine is the main risk factor affecting the occurrence and development of nervous system diseases such 
as atherosclerosis, hypertension, dementia and so on[39]. And homocysteine can be catalyzed by cystathionine synthetase, using vitamin B6 as coenzyme, homocysteine condenses with serine to form L-cystathionine, and then produces cysteine and $\alpha$-ketobutyric acid. $\alpha$-ketobutyric acid is then metabolized by tricarboxylic acid to produce glucose for brain metabolism, affecting cognitive function[40].

\section{Conclusions}

In this study, 84 MCI-related biomarkers and 8 metabolic pathways (D-arginine and D-ornithine metabolism; alanine, aspartic acid and glutamate metabolism; phenylalanine metabolism; arginine and proline metabolism; valine, leucine and isoleucine biosynthesis; cysteine and methionine metabolism; aminoacyl tRNA biosynthesis; D-glutamine and D-glutamate metabolism). The mechanism of SCPE intervention on brain metabolism of MCI was discussed with low, middle and high doses. It was found that different doses of SCPE could call back 36 same metabolic markers and 3 common metabolic pathways (phenylalanine metabolism; alanine, aspartic acid and glutamate metabolism; D-glutamine and D-glutamate metabolism). And with the increase of dose, the number of SCPE callback metabolic markers gradually increased (LD:40; MD:48; HD:54), and the types of main metabolic pathways also changed to a certain extent (the biosynthesis of valine, leucine and isoleucine in low dose changed to cysteine and methionine metabolic pathway in middle and high dose) which may be related to the effect of aminoacyl tRNA. The results above indicate the stability and reliability of 7-month-old SAMP8 mice as a MCI model, and demonstrate the effectiveness of SCPE in the treatment of MCI. Compared with the MD and LD groups, the results showed that the high dose SCPE group has more advantages.

To sum up, this study is of great significance for in-depth understanding of brain metabolomics in mice with MCI and exploring the metabolic mechanism of SCPE in the intervention of MCI. It also provides a new strategy for further understanding the therapeutic mechanism of traditional Chinese medicine. 


\section{Abbreviations}

The following abbreviations are used in this manuscript:

$\begin{array}{ll}\mathrm{A} \beta & \beta \text {-amyloid } \\ \mathrm{A} \beta & \beta \text {-amyloid } \\ \mathrm{AD} & \text { Alzheimer's disease } \\ \text { Cont } & \text { Blank group } \\ \text { FC } & \text { Fold change } \\ \text { FC analysis } & \text { Fold change analysis } \\ \text { HD } & \text { High dose group } \\ \text { LD } & \text { Low dose group } \\ \text { MCI } & \text { Mild cognitive impairment } \\ \text { MD } & \text { Medium dose group } \\ \text { Mean } \pm \text { SD } & \text { Mean } \pm \text { standard deviation } \\ \text { Mod } & \text { Model group } \\ \text { PCA } & \text { Principal Component Analysis } \\ \text { QC-RSC } & \text { Quality control-based robust LOESS signal correction } \\ \text { SAMP8 } & \text { Senescence-accelerated P8 } \\ \text { SCPE } & \text { Sagacious confucius' pillow elixir }\end{array}$

Ethics approval and consent to participate

The experimental protocol was established, according to the ethical guidelines of the Helsinki Declaration and was approved by the Ethics Committee of Heilongjiang University of Traditional Chinese Medicine.

Consent for publication

Not applicable.

Availability of data and materials

The datasets used and analysed during the current study available from the corresponding author on reasonable request.

Competing interests

The authors declare that they have no competing interests.

Funding

This work was supported by the National Natural Sciences Foundation of China (Grant No. 81904307), the Natural Science Foundation of Heilongjiang University of Chinese Medicine (Grant No. 201838), and the Young and Middle-aged Teachers Scientific Research Project of Heilongjiang University of Chinese Medicine (Grant No. 2017001).

Author's contributions

Designed the experiments: JC, ZS and HS. Performed the experiments: ZH, MW, TM, YZ, LS, and XS. Analyzed the data and revised the manuscript: $\mathrm{CH}, \mathrm{QW}, \mathrm{YL}, \mathrm{AS}$ and $\mathrm{ZH}$. All authors discussed the results and commented on the manuscript. All authors approved the final version of manuscript for submission.

\section{Acknowledgements}

Not Applicable.

\section{Author details}

${ }^{1}$ College of Basic Medical and Sciences, Heilongjiang University of Chinese Medicine, 150040 Harbin, Heilongjiang,

China. ${ }^{2}$ Key Laboratory of Chinese Internal Medicine of the Ministry of Education, Dongzhimen Hospital Affiliated with Beijing University of Chinese Medicine, 100700 Beijing, China. ${ }^{3}$ School of Acupuncture-Moxibustion and Tuina, Beijing University of Chinese Medicine, 100029 Beijing, China.

\section{References}

1. Andrés, P., Vico, H., Yáñez, A., Siquier, A., Ferrer, G.A.: Quantifying memory deficits in amnestic mild cognitive impairment. Alzhmer's and Dementia 11, 108-114 (2019)

2. Miotto, E.C., Batista, A.X., Simon, S.S., Hampstead, B.M.: Neurophysiologic and cognitive changes arising from cognitive training interventions in persons with mild cognitive impairment: A systematic review. Neural Plasticity 2018 (2018)

3. Abd Razak, M.A., Ahmad, N.A., Chan, Y.Y., Mohamad Kasim, N., Yusof, M., Abdul Ghani, M.K.A., Omar, M., Abd Aziz, F.A., Jamaluddin, R.: Validity of screening tools for dementia and mild cognitive impairment among the elderly in primary health care: a systematic review. Public Health 169, 84-92 (2019) 
4. Du, L.N., Xie, T., Xu, J.Y., Kang, A., Di, L.Q., Shan, J.J., Wang, S.C.: A metabolomics approach to studying the effects of jinxin oral liquid on rsv-infected mice using uplc/ltq-orbitrap mass spectrometry. Journal of ethnopharmacology 174, 25-36 (2015)

5. Hou, Z.T., Han, Y.S., Liu, Y.T., Xing, Y., Wang, M., Chen, J.: Effect of scpe on cognitive function of type 2 diabetic rats. Chinese Journal of traditional Chinese Medicine 43(22), 4506-4512 (2018)

6. Zheng, Y., Yang, Y., Dong, B., Zheng, H., Lin, X., Du, Y., Li, X., Zhao, L., Gao, H.: Metabonomic profiles delineate potential role of glutamate-glutamine cycle in $\mathrm{db} / \mathrm{db}$ mice with diabetes-associated cognitive decline. Molecular Brain 9(1), 40 (2016)

7. Xie, X.L., Nie, X., Wu, J., Zhang, F., Zhao, L.L., Lin, Y.L., Yin, Y.J., Liu, H., Shu, Y.N., Miao, S.B., Li, H., Chen, P., Han, M.: Smooth muscle $22 \alpha$ facilitates angiotensin ii-induced signaling and vascular contraction. Journal of Molecular Medicine 93(5), 547-558 (2015)

8. Zhu, Z., Xie, Y., Guan, W., Gao, Y., Huang, R., Xia, S., Jian, W., Liang, Z., Zheng, J.: Effects of leukotriene d4 nasal challenge on bronchial responsiveness and inflammation in asthmatic patients with allergic rhinitis. Journal of Thoracic Disease 9(2), 271 (2017)

9. Santockyte, R., Kandoussi, H., Chen, W., Zheng, N., Venkatarangan, L., Gan, J., Shen, H., Bonacorsi, S.J., Easter, J., Burrell, R., Zhang, Y.J., Zeng, J.: Lc-ms/ms bioanalysis of plasma 1, 14-tetradecanedioic acid and 1,16 -hexadecanedioic acid as candidate biomarkers for organic anion-transporting polypeptide mediated drug-drug interactions. Bioanalysis 10(18), 1473-1485 (2018)

10. Cho, M.J., Buescher, R.W., Johnson, M., Janes, M.: Inactivation of pathogenic bacteria by cucumber volatiles (e,z)-2,6-nonadienal and (e)-2-nonenal. Journal of Food Protection 67(5), 1014-1016 (2004)

11. Takamiya, T., Nitta, J., Sato, A., Inamura, Y., Kato, N., Inaba, O., Negi, K., Yamato, T., Matsumura, Y., Takahashi, Y., Goya, M., Hirao, K.: Pulmonary vein isolation plus left atrial posterior wall isolation and additional nonpulmonary vein trigger ablation using high-dose isoproterenol for long-standing persistent atrial fibrillation. Journal of Arrhythmia 35(2), 215-222 (2019)

12. Phillips, R.S., Poteh, P., Krajcovic, D., Miller, K.A., Hoover, T.R.: Crystal structure of d-ornithine/d-lysine decarboxylase, a stereoinverting decarboxylase: Implications for substrate specificity and stereospecificity of fold iii decarboxylases. Biochemistry 58(8), 1038-1042 (2019)

13. Sun, S., Sun, D., Yang, L., Han, J., Liu, R., Wang, L.: Dose-dependent effects of intravenous methoxamine infusion during hip-joint replacement surgery on postoperative cognitive dysfunction and blood tnf- $\alpha$ level in elderly patients: a randomized controlled trial. Bmc Anesthesiology 17(1) (2017)

14. Mansilla: Ammonia-nitrogen added to low-crude-protein diets deficient in dispensable amino acid-nitrogen increases the net release of alanine, citrulline, and glutamate post-splanchnic organ metabolism in growing pigs Journal of Nutrition 149(4), 700 (2019)

15. Fletcher, E., Gao, K., Mercurio, K., Ali, M., Baetz, K.: Yeast chemogenomic screen identifies distinct metabolic pathways required to tolerate exposure to phenolic fermentation inhibitors ferulic acid, 4-hydroxybenzoic acid and coniferyl aldehyde. Metabolic Engineering 52, 98-109 (2019)

16. Wen, L., Zang, L., Huang, K., Li, S., Wang, P.G.: Efficient enzymatic synthesis of I-rhamnulose and I-fuculose. Bioorganic \& Medicinal Chemistry Letters 26(3) (2016)

17. Baslow, M.H., Guilfoyle, D.N.: N-acetyl-I-histidine, a prominent biomolecule in brain and eye of poikilothermic vertebrates. Biomolecules 5(2) (2015)

18. Shabani, S., Mirshekar, M.A.: Diosmin is neuroprotective in a rat model of scopolamine-induced cognitive impairment. Biomedecine \& Pharmacotherapie 108, 1376-1383 (2018)

19. Infante, H.G., del Carmen Ovejero Bendito, M., Cámara, C., Evans, L., Hearn, R., Moesgaard, S.: Isotope dilution quantification of ultratrace gamma-glutamyl-se-methylselenocysteine species using hplc with enhanced icp-ms detection by ultrasonic nebulisation or carbon-loaded plasma. Analytical and Bioanalytical Chemistry 390(8), 2099-2106 (2008)

20. Saha, S., Kauffmann, B., Ferrand, Y., Huc, I.: Selective encapsulation of disaccharide xylobiose by an aromatic foldamer helical capsule. Angewandte Chemie International Edition 57(41) (2018)

21. Arkhipov, S.G., Rychkov, D.A., Pugachev, A.M., Boldyreva, E.V.: New hydrophobic I -amino acid salts: maleates of I -leucine, I -isoleucine and I -norvaline. Acta Crystallographica 71(Pt 7), 584-592 (2015)

22. Dubinin, M.V., Vedernikov, A.A., Khoroshavina, E.I., Samartsev, V.N.: Induction of ca2+-dependent cyclosporin a-insensitive nonspecific permeability of the inner membrane of liver mitochondria and cytochrome c release by $\alpha, \omega$-hexadecanedioic acid in media of varying ionic strength. Biochemistry 79(6), 571-576 (2014)

23. Stilling, F., Wolk, A., Religa, D., Leppert, J., Bergkvist, L., Michaelsson, K., Larsson, S.C.: Adipose tissue fatty acid composition and cognitive impairment. Nutrition 54, 153-157 (2018)

24. Bortoluzzi, V.T., Franceschi, I.D.D., Rieger, E., Wannmacher, C.M.D.: Co-administration of creatine plus pyruvate prevents the effects of phenylalanine administration to female rats during pregnancy and lactation on enzymes activity of energy metabolism in cerebral cortex and hippocampus of the offspring. Neurochemical Research 39(8), 1594-1602 (2014)

25. Zhou, J., Yao, N., Wang, S., An, D., Cao, K., Wei, J., Li, N., Zhao, D., Wang, L., Chen, X., Lu, Y.: Fructus gardeniae-induced gastrointestinal injury was associated with the inflammatory response mediated by the disturbance of vitamin b6, phenylalanine, arachidonic acid, taurine and hypotaurine metabolism. Journal of Ethnopharmacology 235, 47-55 (2019)

26. Gonda, I., Davidovich-Rikanati, R., Bar, E., Lev, S., Jhirad, P., Meshulam, Y., Wissotsky, G., Portnoy, V., Burger, J., Schaffer, A.A., Tadmor, Y., Giovannoni, J.J., Fei, Z., Fait, A., Katzir, N., Lewinsohn, E. Differential metabolism of I-phenylalanine in the formation of aromatic volatiles in melon (cucumis melo I.) fruit. Phytochemistry 148, 122-131 (2018)

27. Engskog, M.K.R., Ersson, L., Haglof, J., Arvidsson, T., Pettersson, C., Brittebo, E.: $\beta$-n-methylamino-l-alanine (bmaa) perturbs alanine, aspartate and glutamate metabolism pathways in human neuroblastoma cells as determined by metabolic profiling. Amino Acids 49(5), 905-919 (2017)

28. Nishikawa, Y., Funakoshi, T., Horimatsu, T., Miyamoto, S., Matsubara, T., Yanagita, M., Nakagawa, S. Yonezawa, A., Matsubara, K., Muto, M.: Accumulation of alpha-fluoro-beta-alanine and fluoro mono acetate in 
a patient with 5-fluorouracil-associated hyperammonemia. Cancer Chemotherapy \& Pharmacology 79(3), 629-633 (2017)

29. Saunders, E.C., Ng, W.W., Chambers, J.M., Ng, M., Naderer, T., Kromer, J.O., Likic, V.A., Mcconville, M.J.: Isotopomer profiling of leishmania mexicana promastigotes reveals important roles for succinate fermentation and aspartate uptake in tricarboxylic acid cycle (tca) anaplerosis, glutamate synthesis, and growth. Journal of Biological Chemistry 286(31), 27706-27717 (2011)

30. Che, A.P., Lau, A.Z., Gu, Y.P., Schroeder, M.A., Barry, J., Cunningham, C.H.: Probing the cardiac malate-aspartate shuttle non-invasively using hyperpolarized

$$
1,2-13 c 2
$$

pyruvate. NMR in Biomedicine 31(1) (2017)

31. B, M.A.I.A.: Homology modeling and molecular dynamic simulation of udp-n-acetylmuramoyl- I-alanine- $d$ -glutamate ligase (murd) from mycobacterium tuberculosis h37rv using in silico approach. Computational Biology and Chemistry 78, 116-126 (2019)

32. Katane, M., Ariyoshi, M., Tateishi, S., Koiwai, S., Takaku, K., Nagai, K., Nakayama, K., Saitoh, Y., Miyamoto, T., Sekine, M., Mita, M., Hamase, K., Matoba, S., Homma, H.: Structural and enzymatic properties of mammalian d-glutamate cyclase. Archives of Biochemistry \& Biophysics 654, 10-18 (2018)

33. Moutiez, M., Belin, P., Gondry, M.: Aminoacyl-trna-utilizing enzymes in natural product biosynthesis. Chemical Reviews 117(8), 5578-5618 (2017)

34. Ulrich, E.C., Van der Donk, W.A.: Cameo appearances of aminoacyl-trna in natural product biosynthesis Current Opinion in Chemical Biology 35, 29-36 (2016)

35. Akahoshi, A., Doi, Y., Sisido, M., Watanabe, K., Ohtsuki, T.: Photo-dependent protein biosynthesis using a caged aminoacyl-trna. Bioorganic \& Medicinal Chemistry Letters 24(23), 5369-5372 (2014)

36. Peacock, J.R., Walvoord, R.R., Chang, A.Y., Kozlowski, M.C., Gamper, H., Hou, Y.M.: Amino acid-dependent stability of the acyl linkage in aminoacyl-trna. Rna-a Publication of the Rna Society 20(6), 758-764 (2014)

37. Cvetesic, N., Perona, J.J., Gruic-Sovulj, I.: Kinetic partitioning between synthetic and editing pathways in class i aminoacyl-trna synthetases occurs at both pre-transfer and post-transfer hydrolytic steps. Journal of Biological Chemistry 287(30), 25381-25394 (2012)

38. Ye, Q., Ji, Q.Q., Yan, W., Yang, F., Wang E D", v.n.p.y. journal=Journal of Biological Chemistry: Acetylation of lysine $\epsilon$-amino groups regulates aminoacyl-trna synthetase activity in escherichia coli

39. Jakubowski, H.: Aminoacyl-trna synthetases and the evolution of coded peptide synthesis: the thioester world. FEBS letters 590(4), 469-481 (2016)

40. Okun, J.G., Gan-Schreier, H., Ben-Omran, T., Schmidt, K.V., Fang-Hoffmann, J., Gramer, G., Abdoh, G., Shahbeck, N., Al Rifai, H., Al Khal, A.L., Haege, G., Chiang, C.C., Kasper, D.C., Wilcken, B., Burgard, P., Hoffmann, G.F.: Newborn screening for vitamin b6 non-responsive classical homocystinuria: Systematical evaluation of a two-tier strategy. JIMD Reports 32, 87-94 (2016)

Figures

Figure 1 PCA score plots in the mode of positive and negative ions.

Figure 2 PLS-DA score plots of model analysing.

Figure 3 Volcano plot of differential ion.

Figure 4 VIP map and Heat map of differential ion clustering analysis.

Figure 5 Changes of metabolic markers in the control group and the model group under the positive ion mode. Compared with the blank group, **P-value i 0.01 . 
Figure 6 Changes of metabolic markers in the control group and the model group under the negitive ion mode. Compared with the blank group, **P-value ; 0.01 .

Figure 7 Heat map of differential metabolites cluster analysis under the positive ion mode.

Figure 8 Heat map of differential metabolites cluster analysis under the negitive ion mode.

Figure 9 Metabolic pathway analysis of $\mathrm{MCl}$ biomarkers.

Figure 10 Metabolic pathway enrichment analysis of $\mathrm{MCl}$ biomarkers.

Figure 11 Metabolic pathway network diagram of $\mathrm{MCl}$ mice.

Figure 12 Metabolic biomarkers of $\mathrm{MCl}$ intervented by low dose of SCPE. (A) low dose (LD) SCPE intervention $\mathrm{MCl}$ biomarker intensity histogram, compared with the model group (Mod), *P-value i 0.05 ; **P-value ; 0.01 ; (B) biomarker heat map. The color is proportional to the difference in metabolites (red: up-regulated; blue: down-regulated).

Figure 13 Pathway analysis of biomarkers for $\mathrm{MCl}$ intervention with low dose of SCPE.

Figure 14 Metabolic pathway enrichment analysis of $\mathrm{MCl}$ biomarkers intervened by SCPE.

Figure 15 Metabolic biomarkers of $\mathrm{MCl}$ intervented by medium dose of SCPE. (A) Histogram of biomarker intensity of medium dose (MD) intervention in $\mathrm{MCl}$, compared with the model group (Mod) *P-value i 0.05 ; **P-value i 0.01 ; (B)Heat map of biomarkers (red: up-regulated; blue: down-regulated).

Figure 16 Pathway analysis of biomarkers for $\mathrm{MCl}$ intervention with medium dose of SCPE.

Figure 17 Metabolic pathway enrichment analysis of $\mathrm{MCl}$ biomarkers intervened by SCPE.

Figure 18 Metabolic biomarkers of $\mathrm{MCl}$ intervented by high dose of SCPE. (A) Bar chart of metabolic intensity of biomarkers of $\mathrm{MCl}$ treated with high dose (HD), compared with the model group (Mod), *P-value i 0.05; **P-value ; 0.01; (B) Heat map of biomarkers (red: up-regulated; blue: down-regulated).

Figure 19 Pathway analysis of biomarkers for $\mathrm{MCl}$ intervention with high dose of SCPE.

Figure 20 Metabolic pathway enrichment analysis of $\mathrm{MCI}$ biomarkers intervened by SCPE.

Figure 21 Venn diagram of metabolic markers in different doses of SCPE. (A) Model group (Model), Low dose group (LD), Medium dose group (MD), High dose group (HD) Venn diagram; (B) Low dose group (LD), Medium dose group (MD), High dose group (HD) Venn diagram. 
Figure 22 Combination diagram of metabolites analysis. (A) Thermograms of 36 metabolic markers in common callback of low, medium and high dose SCPE; (B) Pearson correlation analysis of 36 metabolic markers in the common callback of low, middle and high dose SCPE; (C) The pathway map of 36 metabolic markers for the common callback of low, medium and high dose SCPE; (D) Pathway map of 40 metabolic markers in low dose SCPE callback; (E) The pathway map of 48 metabolic markers for medium dose SCPE callback; (F) The pathway map of 54 metabolic markers for high dose SCPE callback.

Figure 23 Metabolic pathway analysis.

Table 1 Results of differential ions screening.

\begin{tabular}{ccccc}
\hline Mode & Group & Diff ion number & Up & Down \\
\hline pos & Cont vs Mod & 313 & 151 & 162 \\
neg & Cont vs Mod & 356 & 151 & 205 \\
\hline
\end{tabular}

Table 2 Identification table of differential ions.

\begin{tabular}{ccccccc}
\hline Mode & Group & Diff ion number & Up (MS) & Down (MS) & Up (MS2) & Down (MS2) \\
\hline pos & Cont vs Mod & 302 & 114 & 77 & 64 & 35 \\
neg & Cont vs Mod & 169 & 88 & 21 & 47 & 13 \\
\hline
\end{tabular}


Table 3: 84 major metabolic markers associated with mild cognitive impairment identified by UHPLC-Q-TOF/MS (positive/negative ion mode).

\begin{tabular}{|c|c|c|c|c|c|c|c|c|c|c|c|}
\hline Mode & No. & Metabolite & Formula & $m / z$ & RT.min. & Adducts & ratio & VIP & ID.1 & KEGG.ID & Trend \\
\hline \multirow[t]{36}{*}{ pos } & 1 & Angiotensin & $\mathrm{C}_{30} \mathrm{H}_{48} \mathrm{~N}_{8} \mathrm{O}_{9}$ & 647.4 & 5.26775 & $\mathrm{M}+\mathrm{H}-\mathrm{H} 2 \mathrm{O}$ & 61.112 & 5.5206 & C15852 & C15852 & $\uparrow$ \\
\hline & 2 & Leukotriene D4 & $\mathrm{C}_{25} \mathrm{H}_{40} \mathrm{~N}_{2} \mathrm{O}_{6} \mathrm{~S}$ & 514.3 & 5.645633 & $\mathrm{M}+\mathrm{NH} 4$ & 28.983 & 5.0035 & HMDB0003080 & C05951 & $\uparrow$ \\
\hline & 3 & Hexadecanedioic acid & $\mathrm{C}_{16} \mathrm{H}_{30} \mathrm{O}_{4}$ & 309.2 & 5.53135 & $\mathrm{M}+\mathrm{Na}$ & 14.281 & 4.2154 & HMDB0000672 & C19615 & $\uparrow$ \\
\hline & 4 & $(3 Z, 6 Z)-3,6-$ Nonadienal & $\mathrm{C}_{9} \mathrm{H}_{14} \mathrm{O}$ & 139.1 & 6.216 & $\mathrm{M}+\mathrm{H}$ & 12.832 & 4.1182 & HMDB0031152 & C16323 & $\uparrow$ \\
\hline & 5 & Isoproterenol & $\mathrm{C}_{11} \mathrm{H}_{17} \mathrm{NO}_{3}$ & 229.2 & 3.67795 & $\mathrm{M}+\mathrm{NH} 4$ & 10.563 & 3.6591 & HMDB0015197 & C07056 & $\uparrow$ \\
\hline & 6 & D-Ornithine & $\mathrm{C}_{5} \mathrm{H}_{12} \mathrm{~N}_{2} \mathrm{O}_{2}$ & 115.1 & 0.64785 & $\mathrm{M}+\mathrm{H}-\mathrm{H} 2 \mathrm{O}$ & 9.8904 & 3.6995 & C00515 & C00515 & $\uparrow$ \\
\hline & 7 & Methoxamine & $\mathrm{C}_{11} \mathrm{H}_{17} \mathrm{NO}_{3}$ & 229.2 & 4.0559 & $\mathrm{M}+\mathrm{NH} 4$ & 8.4959 & 3.4573 & HMDB0014861 & C07513 & $\uparrow$ \\
\hline & 8 & Citrulline & $\mathrm{C}_{6} \mathrm{H}_{13} \mathrm{~N}_{3} \mathrm{O}_{3}$ & 214.1 & 0.64785 & $M+K$ & 8.3406 & 3.6848 & HMDB0000904 & C00327 & $\uparrow$ \\
\hline & 9 & Coniferyl aldehyde & $\mathrm{C}_{10} \mathrm{H}_{10} \mathrm{O}_{3}$ & 201.1 & 0.740017 & $\mathrm{M}+\mathrm{Na}$ & 8.207 & 3.2201 & C02666 & C02666 & $\uparrow$ \\
\hline & 10 & Isopropylmaleate & $\mathrm{C}_{7} \mathrm{H}_{10} \mathrm{O}_{4}$ & 176.1 & 0.825733 & $\mathrm{M}+\mathrm{NH} 4$ & 6.6125 & 3.1223 & HMDB0012241 & C02631 & $\uparrow$ \\
\hline & 11 & Porphobilinogen & $\mathrm{C}_{10} \mathrm{H}_{14} \mathrm{~N}_{2} \mathrm{O}_{4}$ & 244.1 & 0.725733 & $\mathrm{M}+\mathrm{NH} 4$ & 4.9492 & 3.1194 & C00931 & C00931 & $\uparrow$ \\
\hline & 12 & 11Z-Eicosenoic acid & $\mathrm{C}_{20} \mathrm{H}_{38} \mathrm{O}_{2}$ & 311.3 & 10.8873 & $\mathrm{M}+\mathrm{H}$ & 4.33 & 2.8575 & HMDB0002231 & C16526 & $\uparrow$ \\
\hline & 13 & Piperidine & $\mathrm{C}_{5} \mathrm{H}_{11} \mathrm{~N}$ & 86.1 & 0.825733 & $\mathrm{M}+\mathrm{H}$ & 4.1153 & 2.8547 & HMDB0034301 & C01746 & $\uparrow$ \\
\hline & 14 & L-Isoleucine & $\mathrm{C}_{6} \mathrm{H}_{13} \mathrm{NO}_{2}$ & 132.1 & 2.180333 & $\mathrm{M}+\mathrm{H}$ & 4.0919 & 2.7451 & HMDB0000172 & C00407 & $\uparrow$ \\
\hline & 15 & D-Phenylalanine & $\mathrm{C}_{9} \mathrm{H}_{11} \mathrm{NO}_{2}$ & 148.1 & 3.9559 & $\mathrm{M}+\mathrm{H}-\mathrm{H} 2 \mathrm{O}$ & 4.022 & 2.5231 & C02265 & C02265 & $\uparrow$ \\
\hline & 16 & Topotecan & $\mathrm{C}_{23} \mathrm{H}_{23} \mathrm{~N}_{3} \mathrm{O}_{5}$ & 444.2 & 0.840017 & $\mathrm{M}+\mathrm{Na}$ & 3.9278 & 2.538 & HMDB0015164 & C11158 & $\uparrow$ \\
\hline & 17 & Toluene & $\mathrm{C}_{7} \mathrm{H}_{8}$ & 93.07 & 3.6923 & $\mathrm{M}+\mathrm{H}$ & 3.6764 & 2.5385 & HMDB0034168 & C01455 & $\uparrow$ \\
\hline & 18 & 2-Phenylacetamide & $\mathrm{C}_{8} \mathrm{H}_{9} \mathrm{NO}$ & 136.1 & 0.825733 & $\mathrm{M}+\mathrm{H}$ & 3.5094 & 2.5669 & HMDB0010715 & C02505 & $\uparrow$ \\
\hline & 19 & L-Glutamic acid & $\mathrm{C}_{5} \mathrm{H}_{9} \mathrm{NO}_{4}$ & 148.1 & 2.308917 & $\mathrm{M}+\mathrm{H}$ & 3.465 & 2.3271 & HMDB0000148 & C00025 & $\uparrow$ \\
\hline & 20 & 4-Hydroxycinnamic acid & $\mathrm{C}_{9} \mathrm{H}_{8} \mathrm{O}_{3}$ & 182.1 & 1.931017 & $\mathrm{M}+\mathrm{NH} 4$ & 3.2223 & 2.3325 & HMDB0002035 & C00811 & $\uparrow$ \\
\hline & 21 & Erucic acid & $\mathrm{C}_{22} \mathrm{H}_{42} \mathrm{O}_{2}$ & 356.4 & 8.41255 & $\mathrm{M}+\mathrm{NH} 4$ & 3.0437 & 2.6268 & HMDB0002068 & $\mathrm{C} 08316$ & $\uparrow$ \\
\hline & 22 & Aminocaproic acid & $\mathrm{C}_{6} \mathrm{H}_{13} \mathrm{NO}_{2}$ & 132.1 & 0.825733 & $\mathrm{M}+\mathrm{H}$ & 2.8944 & 2.3466 & HMDB0001901 & C02378 & $\uparrow$ \\
\hline & 23 & 1-Phenyl-1,2-propanedione & $\mathrm{C}_{9} \mathrm{H}_{8} \mathrm{O}_{2}$ & 166.1 & 1.3322 & $\mathrm{M}+\mathrm{NH} 4$ & 2.8934 & 2.2699 & C17268 & C17268 & $\uparrow$ \\
\hline & 24 & trans-Cinnamic acid & $\mathrm{C}_{9} \mathrm{H}_{8} \mathrm{O}_{2}$ & 166.1 & 3.67795 & $\mathrm{M}+\mathrm{NH} 4$ & 2.7338 & 2.2063 & C00423 & C00423 & $\uparrow$ \\
\hline & 25 & Eicosadienoic acid & $\mathrm{C}_{20} \mathrm{H}_{36} \mathrm{O}_{2}$ & 331.3 & 9.264467 & $\mathrm{M}+\mathrm{Na}$ & 2.6525 & 2.284 & HMDB0005060 & C16525 & $\uparrow$ \\
\hline & 26 & Norepinephrine & $\mathrm{C}_{8} \mathrm{H}_{11} \mathrm{NO}_{3}$ & 187.1 & 0.711433 & $\mathrm{M}+\mathrm{NH} 4$ & 2.428 & 1.9473 & HMDB0000216 & C00547 & $\uparrow$ \\
\hline & 27 & Enol-phenylpyruvate & $\mathrm{C}_{9} \mathrm{H}_{8} \mathrm{NO}_{3}$ & 182.1 & 0.825733 & $\mathrm{M}+\mathrm{NH} 4$ & 2.2705 & 1.8435 & HMDB0012225 & C02763 & $\uparrow$ \\
\hline & 28 & Phenylacetaldehyde & $\mathrm{C}_{8} \mathrm{H}_{8} \mathrm{O}$ & 121.1 & 0.825733 & $\mathrm{M}+\mathrm{H}$ & 1.9547 & 1.6372 & HMDB0006236 & C00601 & $\uparrow$ \\
\hline & 29 & o-Cresol & $\mathrm{C}_{7} \mathrm{H}_{8} \mathrm{O}$ & 91.06 & 3.6923 & $\mathrm{M}+\mathrm{H}-\mathrm{H} 2 \mathrm{O}$ & 1.7744 & 1.483 & HMDB0002055 & C01542 & $\uparrow$ \\
\hline & 30 & L-Cystathionine & $\mathrm{C}_{7} \mathrm{H}_{14} \mathrm{~N}_{2} \mathrm{O}_{4} \mathrm{~S}$ & 205.1 & 0.825733 & $\mathrm{M}+\mathrm{H}-\mathrm{H} 2 \mathrm{O}$ & 1.69 & 1.0319 & HMDB0000099 & C02291 & $\uparrow$ \\
\hline & 31 & 5-Hydroxy-L-tryptophan & $\mathrm{C}_{11} \mathrm{H}_{12} \mathrm{~N}_{2} \mathrm{O}_{3}$ & 203.1 & 0.825733 & $\mathrm{M}+\mathrm{H}-\mathrm{H} 2 \mathrm{O}$ & 1.6491 & 1.4414 & HMDB0000472 & C00643 & $\uparrow$ \\
\hline & 32 & L-Carnitine & $\mathrm{C}_{7} \mathrm{H}_{15} \mathrm{NO}_{3}$ & 162.1 & 0.633567 & $\mathrm{M}+\mathrm{H}$ & 1.4357 & 1.389 & HMDB0000062 & C00318 & $\uparrow$ \\
\hline & 33 & 4-Hydroxyifosfamide & $\mathrm{C}_{7} \mathrm{H}_{15} \mathrm{Cl}_{2} \mathrm{~N}_{2} \mathrm{O}_{3} \mathrm{P}$ & 259 & 5.559917 & $\mathrm{M}+\mathrm{H}-\mathrm{H} 2 \mathrm{O}$ & 0.7513 & 1.1344 & C16553 & C16553 & $\downarrow$ \\
\hline & 34 & Alpha-Tocotrienol & $\mathrm{C}_{29} \mathrm{H}_{44} \mathrm{O}_{2}$ & 425.3 & 11.16025 & $\mathrm{M}+\mathrm{H}$ & 0.6676 & 1.1892 & HMDB0006327 & C14153 & $\downarrow$ \\
\hline & 35 & Allyl isothiocyanate & $\mathrm{C}_{4} \mathrm{H}_{5} \mathrm{NS}$ & 100 & 0.825733 & $\mathrm{M}+\mathrm{H}$ & 0.6049 & 1.4158 & HMDB0005843 & C19317 & $\downarrow$ \\
\hline & 36 & L-Aspartic acid & $\mathrm{C}_{4} \mathrm{H}_{7} \mathrm{NO}_{4}$ & 134 & 0.81145 & $\mathrm{M}+\mathrm{H}$ & 0.582 & 1.814 & HMDB0000191 & C00049 & $\downarrow$ \\
\hline
\end{tabular}




\begin{tabular}{|c|c|c|c|c|c|c|c|c|c|c|c|}
\hline Mode & No. & Metabolite & Formula & $m / z$ & RT.min. & Adducts & ratio & VIP & ID.1 & KEGG.ID & Trend \\
\hline & 37 & 2-Aminoacrylic acid & $\mathrm{C}_{3} \mathrm{H}_{5} \mathrm{NO}_{2}$ & 88.04 & 0.825733 & $\mathrm{M}+\mathrm{H}$ & 0.5131 & 2.0989 & HMDB0003609 & $\mathrm{C} 02218$ & $\downarrow$ \\
\hline & 38 & Mannitol 1-phosphate & $\mathrm{C}_{6} \mathrm{H}_{15} \mathrm{O}_{9} \mathrm{P}$ & 245 & 0.66215 & $\mathrm{M}+\mathrm{H}-\mathrm{H} 2 \mathrm{O}$ & 0.4915 & 1.6516 & HMDB0001530 & $\mathrm{C} 00644$ & $\downarrow$ \\
\hline & 39 & Boldione & $\mathrm{C}_{19} \mathrm{H}_{24} \mathrm{O}_{2}$ & 307.2 & 5.46775 & $\mathrm{M}+\mathrm{Na}$ & 0.4217 & 1.7867 & HMDB0003422 & C20144 & $\downarrow$ \\
\hline & 40 & N-Acetyl-L-aspartic acid & $\mathrm{C}_{6} \mathrm{H}_{9} \mathrm{NO}_{5}$ & 158 & 0.81145 & $\mathrm{M}+\mathrm{H}-\mathrm{H} 2 \mathrm{O}$ & 0.3781 & 2.3555 & HMDB0000812 & $\mathrm{C} 01042$ & $\downarrow$ \\
\hline & 41 & Episterol & $\mathrm{C}_{28} \mathrm{H}_{46} \mathrm{O}$ & 381.4 & 11.68417 & $\mathrm{M}+\mathrm{H}-\mathrm{H} 2 \mathrm{O}$ & 0.3379 & 2.0687 & HMDB0006847 & C15777 & $\downarrow$ \\
\hline & 42 & Cytosine & $\mathrm{C}_{4} \mathrm{H}_{5} \mathrm{~N}_{3} \mathrm{O}$ & 112.1 & 0.725733 & $\mathrm{M}+\mathrm{H}$ & 0.3143 & 2.7065 & $\mathrm{C} 00380$ & $\mathrm{C} 00380$ & $\downarrow$ \\
\hline & 43 & 2-Arachidonylglycerol & $\mathrm{C}_{23} \mathrm{H}_{38} \mathrm{O}_{4}$ & 396.3 & 7.627967 & $\mathrm{M}+\mathrm{NH} 4$ & 0.1923 & 2.7597 & HMDB0004666 & C13856 & $\downarrow$ \\
\hline & 44 & LysoSM(d18:1) & $\mathrm{C}_{23} \mathrm{H}_{50} \mathrm{~N}_{2} \mathrm{O}_{5} \mathrm{P}$ & 466.4 & 7.93465 & $\mathrm{M}+\mathrm{H}$ & 0.0376 & 4.7106 & HMDB0006482 & $\mathrm{C} 03640$ & $\downarrow$ \\
\hline & 45 & Taurochenodesoxycholic acid & $\mathrm{C}_{26} \mathrm{H}_{45} \mathrm{NO}_{6} \mathrm{~S}$ & 517.3 & 6.565317 & $\mathrm{M}+\mathrm{NH} 4$ & 0.035 & 4.4026 & HMDB0000951 & $\mathrm{C} 05465$ & $\downarrow$ \\
\hline \multirow[t]{28}{*}{ neg } & 46 & L-Rhamnulose & $\mathrm{C}_{6} \mathrm{H}_{12} \mathrm{O}_{5}$ & 199 & 0.725683 & $\mathrm{M}+\mathrm{Cl}$ & 50.831 & 5.8453 & C00861 & C00861 & $\uparrow$ \\
\hline & 47 & N-Acetyl-L-histidine & $\mathrm{C}_{8} \mathrm{H}_{11} \mathrm{~N}_{3} \mathrm{O}_{3}$ & 196.1 & 0.725683 & $\mathrm{M}-\mathrm{H}$ & 31.585 & 5.2531 & C02997 & $\mathrm{C} 02997$ & $\uparrow$ \\
\hline & 48 & Diosmin & $\mathrm{C}_{28} \mathrm{H}_{32} \mathrm{O}_{15}$ & 607.2 & 0.8257 & $\mathrm{M}-\mathrm{H}$ & 13.039 & 4.806 & C10039 & C10039 & $\uparrow$ \\
\hline & 49 & Argininic acid & $\mathrm{C}_{6} \mathrm{H}_{13} \mathrm{~N}_{3} \mathrm{O}_{3}$ & 174.1 & 0.647817 & $\mathrm{M}-\mathrm{H}$ & 6.585 & 3.6599 & HMDB0003148 & null & $\uparrow$ \\
\hline & 50 & Ornithine & $\mathrm{C}_{5} \mathrm{H}_{12} \mathrm{~N}_{2} \mathrm{O}_{2}$ & 131.1 & 0.647817 & $\mathrm{M}-\mathrm{H}$ & 5.2992 & 3.4788 & C01602 & C01602 & $\uparrow$ \\
\hline & 51 & 2-Isopropylmalic acid & $\mathrm{C}_{7} \mathrm{H}_{12} \mathrm{O}_{5}$ & 211 & 0.61925 & $\mathrm{M}+\mathrm{Cl}$ & 5.1237 & 3.422 & HMDB0000402 & $\mathrm{C} 02504$ & $\uparrow$ \\
\hline & 52 & L-Leucine & $\mathrm{C}_{6} \mathrm{H}_{13} \mathrm{NO}_{2}$ & 130.1 & 2.108883 & $\mathrm{M}-\mathrm{H}$ & 4.9642 & 3.2231 & HMDB0000687 & $\mathrm{C} 00123$ & $\uparrow$ \\
\hline & 53 & gamma-Glutamyl-Se-methyl- selenocysteine & $\mathrm{C}_{9} \mathrm{H}_{16} \mathrm{~N}_{2} \mathrm{O}_{5} \mathrm{Se}$ & 311 & 3.692283 & $\mathrm{M}-\mathrm{H}$ & 4.3956 & 3.126 & C05695 & C05695 & $\uparrow$ \\
\hline & 54 & Xylobiose & $\mathrm{C}_{10} \mathrm{H}_{18} \mathrm{O}_{9}$ & 281.1 & 0.576383 & $\mathrm{M}-\mathrm{H}$ & 4.3586 & 3.1162 & $\mathrm{C} 01630$ & $\mathrm{C} 01630$ & $\uparrow$ \\
\hline & 55 & L-Norleucine & $\mathrm{C}_{6} \mathrm{H}_{13} \mathrm{NO}_{2}$ & 130.1 & 0.839983 & $\mathrm{M}-\mathrm{H}$ & 4.2301 & 3.2557 & HMDB0001645 & C01933 & $\uparrow$ \\
\hline & 56 & beta-Tyrosine & $\mathrm{C}_{9} \mathrm{H}_{11} \mathrm{NO}_{3}$ & 180.1 & 0.839983 & $\mathrm{M}-\mathrm{H}$ & 3.9327 & 2.4783 & C04368 & $\mathrm{C} 04368$ & $\uparrow$ \\
\hline & 57 & $\begin{array}{l}\text { 3-Hydroxy-4-hydroxymethyl-2-methyl- } \\
\text { carboxylate }\end{array}$ & $\mathrm{C}_{8} \mathrm{H}_{9} \mathrm{NO}_{4}$ & 182 & 0.8257 & $\mathrm{M}-\mathrm{H}$ & 3.5571 & 2.6123 & C04773 & C04773 & $\uparrow$ \\
\hline & 58 & Phenoxybenzamine & $\mathrm{C}_{18} \mathrm{H}_{22} \mathrm{CINO}$ & 302.1 & 0.839983 & $\mathrm{M}-\mathrm{H}$ & 3.5439 & 2.6334 & HMDB0015061 & $\mathrm{C} 07435$ & $\uparrow$ \\
\hline & 59 & 4-Hydroxy-4-(3-pyridyl)-butanoic acid & $\mathrm{C}_{9} \mathrm{H}_{11} \mathrm{O}_{3}$ & 180.1 & 1.945267 & $\mathrm{M}-\mathrm{H}$ & 3.5409 & 2.7072 & HMDB0001119 & C19579 & $\uparrow$ \\
\hline & 60 & L-Phenylalanine & $\mathrm{C}_{9} \mathrm{H}_{11} \mathrm{NO}_{2}$ & 164.1 & 3.678 & $\mathrm{M}-\mathrm{H}$ & 3.4942 & 2.8079 & HMDB0000159 & $\mathrm{C} 00079$ & $\uparrow$ \\
\hline & 61 & Benzocaine & $\mathrm{C}_{9} \mathrm{H}_{11} \mathrm{NO}_{2}$ & 164.1 & 1.9167 & $\mathrm{M}-\mathrm{H}$ & 3.4863 & 2.1073 & C07527 & C07527 & $\uparrow$ \\
\hline & 62 & L-Valine & $\mathrm{C}_{5} \mathrm{H}_{11} \mathrm{NO}_{2}$ & 116.1 & 0.76855 & $\mathrm{M}-\mathrm{H}$ & 3.4154 & 2.9604 & C00183 & $\mathrm{C} 00183$ & $\uparrow$ \\
\hline & 63 & N6-Acetyl-L-lysine & $\mathrm{C}_{8} \mathrm{H}_{16} \mathrm{~N}_{2} \mathrm{O}_{3}$ & 223.1 & 0.8257 & $\mathrm{M}+\mathrm{Cl}$ & 3.378 & 2.6149 & HMDB0000206 & $\mathrm{C} 02727$ & $\uparrow$ \\
\hline & 64 & 5-Hydroxyindoleacetate & $\mathrm{C}_{10} \mathrm{H}_{9} \mathrm{NO}_{3}$ & 190.1 & 4.319617 & $\mathrm{M}-\mathrm{H}$ & 3.2636 & 2.9245 & C05635 & C05635 & $\uparrow$ \\
\hline & 65 & L-Asparagine & $\mathrm{C}_{4} \mathrm{H}_{8} \mathrm{~N}_{2} \mathrm{O}_{3}$ & 131 & 0.590667 & $\mathrm{M}-\mathrm{H}$ & 2.5767 & 2.5534 & HMDB0000168 & $\mathrm{C} 00152$ & $\uparrow$ \\
\hline & 66 & (S)-2-(Hydroxymethyl)glutarate & $\mathrm{C}_{6} \mathrm{H}_{10} \mathrm{O}_{5}$ & 161 & 0.839983 & $\mathrm{M}-\mathrm{H}$ & 2.4021 & 2.6044 & C16390 & C16390 & $\uparrow$ \\
\hline & 67 & Paraquat dichloride & $\mathrm{C}_{12} \mathrm{H}_{14} \mathrm{Cl}_{2} \mathrm{~N}_{2}$ & 255 & 0.8257 & $\mathrm{M}-\mathrm{H}$ & 2.0974 & 2.3268 & $\mathrm{C} 00225$ & $\mathrm{C} 00225$ & $\uparrow$ \\
\hline & 68 & Oxypurinol & $\mathrm{C}_{5} \mathrm{H}_{4} \mathrm{~N}_{4} \mathrm{O}_{2}$ & 151 & 0.8257 & $\mathrm{M}-\mathrm{H}$ & 2.0833 & 1.4448 & HMDB0000786 & C07599 & $\uparrow$ \\
\hline & 69 & Methdilazine & $\mathrm{C}_{18} \mathrm{H}_{20} \mathrm{~N}_{2} \mathrm{~S}$ & 331.1 & 7.278317 & $\mathrm{M}+\mathrm{Cl}$ & 1.9345 & 1.5244 & C07175 & C07175 & $\uparrow$ \\
\hline & 70 & 5-(4-Acetoxy-1-butynyl)-2,2'-bithiophene & $\mathrm{C}_{14} \mathrm{H}_{12} \mathrm{O}_{2} \mathrm{~S}_{2}$ & 275 & 0.725683 & $\mathrm{M}-\mathrm{H}$ & 1.8312 & 1.4485 & HMDB0034454 & $\mathrm{C} 04485$ & $\uparrow$ \\
\hline & 71 & Gibberellin A15 open lactone & $\mathrm{C}_{20} \mathrm{H}_{28} \mathrm{O}_{5}$ & 347.2 & 8.062867 & $\mathrm{M}-\mathrm{H}$ & 1.7504 & 1.3783 & C11860 & C11860 & $\uparrow$ \\
\hline & 72 & N1-Acetylspermine & $\mathrm{C}_{12} \mathrm{H}_{28} \mathrm{~N}_{4} \mathrm{O}$ & 279.2 & 8.062867 & $\mathrm{M}+\mathrm{Cl}$ & 1.7265 & 1.2824 & HMDB0001186 & $\mathrm{C} 02567$ & $\uparrow$ \\
\hline & 73 & Thorium-232 & Th & 231 & 0.811417 & $\mathrm{M}-\mathrm{H}$ & 1.6498 & 1.4663 & C19157 & C19157 & $\uparrow$ \\
\hline
\end{tabular}




\begin{tabular}{|c|c|c|c|c|c|c|c|c|c|c|c|}
\hline Mode & No. & Metabolite & Formula & $\mathrm{m} / \mathrm{z}$ & RT.min. & Adducts & ratio & VIP & ID.1 & KEGG.ID & Trend \\
\hline & 74 & L-Malic acid & $\mathrm{C}_{4} \mathrm{H}_{6} \mathrm{O}_{5}$ & 133 & 0.7114 & $\mathrm{M}-\mathrm{H}$ & 1.5318 & 1.266 & HMDB0000156 & C00149 & $\uparrow$ \\
\hline & 75 & Triamcinolone diacetate & $\mathrm{C}_{25} \mathrm{H}_{31} \mathrm{FO}_{8}$ & 477.2 & 10.08828 & $\mathrm{M}-\mathrm{H}$ & 1.4132 & 1.5431 & C08184 & C08184 & $\uparrow$ \\
\hline & 76 & Telmisartan & $\mathrm{C}_{33} \mathrm{H}_{30} \mathrm{~N}_{4} \mathrm{O}_{2}$ & 513.2 & 10.08828 & $\mathrm{M}-\mathrm{H}$ & 1.4056 & 1.5119 & HMDB0015101 & C07710 & $\uparrow$ \\
\hline & 77 & 16-Hydroxy hexadecanoic acid & $\mathrm{C}_{16} \mathrm{H}_{32} \mathrm{O}_{3}$ & 271.2 & 9.317567 & $\mathrm{M}-\mathrm{H}$ & 0.7498 & 1.0048 & HMDB0006294 & C18218 & $\downarrow$ \\
\hline & 78 & Ricinoleic acid & $\mathrm{C}_{18} \mathrm{H}_{34} \mathrm{O}_{3}$ & 297.2 & 8.733233 & $\mathrm{M}-\mathrm{H}$ & 0.7007 & 1.2711 & C08365 & C08365 & $\downarrow$ \\
\hline & 79 & L-Arabinose & $\mathrm{C}_{5} \mathrm{H}_{10} \mathrm{O}_{5}$ & 185 & 0.7114 & $\mathrm{M}+\mathrm{Cl}$ & 0.6415 & 1.4298 & HMDB0000646 & C00259 & $\downarrow$ \\
\hline & 80 & Panaxytriol & $\mathrm{C}_{17} \mathrm{H}_{26} \mathrm{O}_{3}$ & 277.2 & 7.962867 & $\mathrm{M}-\mathrm{H}$ & 0.5289 & 1.735 & C16792 & C16792 & $\downarrow$ \\
\hline & 81 & 3-Deoxy-D-manno-octulosonate 8-phosphate & $\mathrm{C}_{8} \mathrm{H}_{15} \mathrm{O}_{11} \mathrm{P}$ & 353 & 0.7114 & $\mathrm{M}+\mathrm{Cl}$ & 0.4685 & 2.4592 & C04478 & C04478 & $\downarrow$ \\
\hline & 82 & $\left(2^{\prime} \mathrm{E}, 4^{\prime} \mathrm{Z}, 8 \mathrm{E}\right)$-Colneleic acid & $\mathrm{C}_{18} \mathrm{H}_{30} \mathrm{O}_{3}$ & 293.2 & 8.790383 & $\mathrm{M}-\mathrm{H}$ & 0.2308 & 4.0805 & HMDB0030995 & C19827 & $\downarrow$ \\
\hline & 83 & GDP-glucose & $\mathrm{C}_{16} \mathrm{H}_{25} \mathrm{~N}_{5} \mathrm{O}_{16} \mathrm{P}_{2}$ & 604.1 & 0.839983 & $\mathrm{M}-\mathrm{H}$ & 0.2206 & 2.9012 & C00394 & C00394 & $\downarrow$ \\
\hline & 84 & Juvenile hormone III & $\mathrm{C}_{16} \mathrm{H}_{26} \mathrm{O}_{3}$ & 265.2 & 7.92 & $\mathrm{M}-\mathrm{H}$ & 0.1109 & 4.5665 & C09694 & C09694 & $\downarrow$ \\
\hline
\end{tabular}

Note: $\uparrow$ represents up-regulation; $\downarrow$ represents down-regulation. 
Table 4: Information of metabolic pathways.

\begin{tabular}{|c|c|c|c|c|c|c|c|c|c|}
\hline No. & Pathway Name & Total & Hits & Expected & Raw $p$ & $-\log (p)$ & Holm adjust & FDR & Impact \\
\hline 1 & D-Arginine and D-ornithine metabolism & 8 & 2 & 0.26921 & 0.02746 & 3.5952 & 1 & 0.36607 & 0.50000 \\
\hline 2 & Alanine, aspartate and glutamate metabolism & 24 & 4 & 0.80764 & 0.00757 & 4.8837 & 0.59035 & 0.17868 & 0.48718 \\
\hline 3 & Phenylalanine metabolism & 45 & 7 & 1.5143 & 0.00061 & 7.4062 & 0.048597 & 0.04860 & 0.35177 \\
\hline 4 & Arginine and proline metabolism & 77 & 4 & 2.5912 & 0.25891 & 1.3513 & 1 & 1 & 0.18535 \\
\hline 5 & Valine, leucine and isoleucine biosynthesis & 27 & 5 & 0.9086 & 0.00172 & 6.3662 & 0.13577 & 0.06875 & 0.15898 \\
\hline 6 & Cysteine and methionine metabolism & 56 & 3 & 1.8845 & 0.29104 & 1.2343 & 1 & 1 & 0.13505 \\
\hline 7 & Aminoacyl-tRNA biosynthesis & 75 & 7 & 2.5239 & 0.01177 & 4.4418 & 0.89485 & 0.18839 & 0.11268 \\
\hline 8 & D-Glutamine and D-glutamate metabolism & 11 & 1 & 0.37017 & 0.31432 & 1.1574 & 1 & 1 & 0.11230 \\
\hline 9 & Drug metabolism - cytochrome P450 & 86 & 1 & 2.8941 & 0.95012 & 0.05117 & 1 & 1 & 0.04839 \\
\hline 10 & Nicotinate and nicotinamide metabolism & 44 & 2 & 1.4807 & 0.43987 & 0.82128 & 1 & 1 & 0.04454 \\
\hline 11 & Citrate cycle (TCA cycle) & 20 & 1 & 0.67304 & 0.49711 & 0.69894 & 1 & 1 & 0.04361 \\
\hline 12 & Fructose and mannose metabolism & 48 & 2 & 1.6153 & 0.48529 & 0.723 & 1 & 1 & 0.03942 \\
\hline 13 & Tyrosine metabolism & 76 & 2 & 2.5575 & 0.73446 & 0.30861 & 1 & 1 & 0.03812 \\
\hline 14 & Ubiquinone and other terpenoid-quinone biosynthesis & 36 & 1 & 1.2115 & 0.71106 & 0.341 & 1 & 1 & 0.03370 \\
\hline 15 & Vitamin B6 metabolism & 32 & 1 & 1.0769 & 0.66801 & 0.40346 & 1 & 1 & 0.02697 \\
\hline 16 & Arachidonic acid metabolism & 62 & 1 & 2.0864 & 0.88354 & 0.12382 & 1 & 1 & 0.02550 \\
\hline 17 & Glyoxylate and dicarboxylate metabolism & 50 & 1 & 1.6826 & 0.82263 & 0.19525 & 1 & 1 & 0.02420 \\
\hline 18 & Valine, leucine and isoleucine degradation & 40 & 3 & 1.3461 & 0.14956 & 1.9001 & 1 & 1 & 0.02232 \\
\hline 19 & Pyrimidine metabolism & 60 & 1 & 2.0191 & 0.87506 & 0.13346 & 1 & 1 & 0.02127 \\
\hline 20 & Tryptophan metabolism & 79 & 1 & 2.6585 & 0.93606 & 0.06607 & 1 & 1 & 0.01143 \\
\hline 21 & Glutathione metabolism & 38 & 2 & 1.2788 & 0.36803 & 0.9996 & 1 & 1 & 0.01095 \\
\hline 22 & Primary bile acid biosynthesis & 47 & 1 & 1.5816 & 0.80303 & 0.21936 & 1 & 1 & 0.00992 \\
\hline 23 & Porphyrin and chlorophyll metabolism & 104 & 2 & 3.4998 & 0.87425 & 0.13439 & 1 & 1 & 0.00954 \\
\hline 24 & Nitrogen metabolism & 39 & 5 & 1.3124 & 0.00893 & 4.7179 & 0.68793 & 0.17868 & 0.00830 \\
\hline 25 & Sphingolipid metabolism & 25 & 1 & 0.8413 & 0.5769 & 0.55009 & 1 & 1 & 0.00191 \\
\hline 26 & Starch and sucrose metabolism & 50 & 1 & 1.6826 & 0.82263 & 0.19525 & 1 & 1 & 0.00149 \\
\hline 27 & Phenylalanine, tyrosine and tryptophan biosynthesis & 27 & 1 & 0.9086 & 0.60519 & 0.50221 & 1 & 1 & 0.00062 \\
\hline 28 & Histidine metabolism & 44 & 2 & 1.4807 & 0.43987 & 0.82128 & 1 & 1 & 0.00051 \\
\hline 29 & Amino sugar and nucleotide sugar metabolism & 88 & 2 & 2.9614 & 0.80562 & 0.21614 & 1 & 1 & 0.00007 \\
\hline 30 & Cyanoamino acid metabolism & 16 & 2 & 0.53843 & 0.099 & 2.3127 & 1 & 1 & 0 \\
\hline 31 & Pantothenate and $\mathrm{CoA}$ biosynthesis & 27 & 2 & 0.9086 & 0.22978 & 1.4706 & 1 & 1 & 0 \\
\hline 32 & Pyruvate metabolism & 32 & 2 & 1.0769 & 0.29305 & 1.2274 & 1 & 1 & 0 \\
\hline 33 & Glycine, serine and threonine metabolism & 48 & 2 & 1.6153 & 0.48529 & 0.723 & 1 & 1 & 0 \\
\hline 34 & Selenoamino acid metabolism & 22 & 1 & 0.74034 & 0.53067 & 0.63362 & 1 & 1 & 0 \\
\hline 35 & beta-Alanine metabolism & 28 & 1 & 0.94225 & 0.61863 & 0.48025 & 1 & 1 & 0 \\
\hline 36 & alpha-Linolenic acid metabolism & 29 & 1 & 0.9759 & 0.63161 & 0.45948 & 1 & 1 & 0 \\
\hline 37 & Lysine biosynthesis & 32 & 1 & 1.0769 & 0.66801 & 0.40346 & 1 & 1 & 0 \\
\hline
\end{tabular}




\begin{tabular}{|c|c|c|c|c|c|c|c|c|c|}
\hline No. & Pathway Name & Total & Hits & Expected & Raw p & $-\log (p)$ & Holm adjust & FDR & Impact \\
\hline 38 & Propanoate metabolism & 35 & 1 & 1.1778 & 0.70084 & 0.35547 & 1 & 1 & 0 \\
\hline 39 & Butanoate metabolism & 40 & 1 & 1.3461 & 0.74859 & 0.28957 & 1 & 1 & 0 \\
\hline 40 & Ascorbate and aldarate metabolism & 45 & 1 & 1.5143 & 0.78879 & 0.23725 & 1 & 1 & 0 \\
\hline 41 & Lysine degradation & 47 & 1 & 1.5816 & 0.80303 & 0.21936 & 1 & 1 & 0 \\
\hline 42 & Pentose and glucuronate interconversions & 53 & 1 & 1.7835 & 0.8403 & 0.17399 & 1 & 1 & 0 \\
\hline
\end{tabular}

Note: the analysis results of pathway analysis are shown in detail in the Table 4. Total/Hits is the number of metabolites enriched / the number of all metabolites in the pathway, Holmadjust is the p value corrected by HB method, FDR is the p value corrected by False Discovery Rate, and Impact is the pathway impact value obtained by pathway topology analysis. In order to more intuitively reflect the importance of participating in $\mathrm{MCl}$ metabolic pathway, this part uses Impact as a factor to enrich and analyze each pathway, in which blue represents the main candidate metabolic pathway, green is the related metabolic pathway, the specific enrichment situation is shown in Figure 10. 
Table 5 List of biomarkers of metabolism for $\mathrm{MCl}$ intervened by low dose of SCPE.

\begin{tabular}{|c|c|c|c|}
\hline Mode & No. & Metabolite & P-value \\
\hline \multirow[t]{22}{*}{ pos } & 1 & Hexadecanedioic acid & $9.98554 \mathrm{E}-05$ \\
\hline & 2 & Eicosadienoic acid & 0.000206941 \\
\hline & 3 & L-Isoleucine & 0.002584405 \\
\hline & 4 & Leukotriene D4 & 0.002641808 \\
\hline & 5 & 11Z-Eicosenoic acid & 0.002793092 \\
\hline & 6 & D-Phenylalanine & 0.003602312 \\
\hline & 7 & Isopropylmaleate & 0.003611113 \\
\hline & 8 & trans-Cinnamic acid & 0.006786435 \\
\hline & 9 & Methoxamine & 0.010427954 \\
\hline & 10 & (3Z,6Z)-3,6-Nonadienal & 0.011802975 \\
\hline & 11 & Isoproterenol & 0.012203182 \\
\hline & 12 & Toluene & 0.01258429 \\
\hline & 13 & o-Cresol & 0.013207123 \\
\hline & 14 & Angiotensin & 0.014740774 \\
\hline & 15 & Boldione & 0.017072285 \\
\hline & 16 & 2-Arachidonylglycerol & 0.020932654 \\
\hline & 17 & Coniferyl aldehyde & 0.022031139 \\
\hline & 18 & 4-Hydroxyifosfamide & 0.025223664 \\
\hline & 19 & L-Glutamic acid & 0.025331093 \\
\hline & 20 & LysoSM(d18:1) & 0.033718971 \\
\hline & 21 & Episterol & 0.037604253 \\
\hline & 22 & Taurochenodesoxycholic acid & 0.049833646 \\
\hline \multirow[t]{18}{*}{ neg } & 23 & N1-Acetylspermine & 0.004099225 \\
\hline & 24 & L-Phenylalanine & 0.004299337 \\
\hline & 25 & Gibberellin A15 open lactone & 0.006128052 \\
\hline & 26 & L-Leucine & 0.010258797 \\
\hline & 27 & 16-Hydroxy hexadecanoic acid & 0.011032448 \\
\hline & 28 & Telmisartan & 0.016892508 \\
\hline & 29 & 5-Hydroxyindoleacetate & 0.017305416 \\
\hline & 30 & L-Rhamnulose & 0.019094889 \\
\hline & 31 & Panaxytriol & 0.020138285 \\
\hline & 32 & 2-Isopropylmalic acid & 0.022017423 \\
\hline & 33 & gamma-Glutamyl-Se-methylselenocysteine & 0.025697097 \\
\hline & 34 & $\mathrm{~N}$-Acetyl-L-histidine & 0.026415795 \\
\hline & 35 & Paraquat dichloride & 0.029581984 \\
\hline & 36 & (S)-2-(Hydroxymethyl)glutarate & 0.033449195 \\
\hline & 37 & $\left(2^{\prime} E, 4^{\prime} Z, 8 E\right)$-Colneleic acid & 0.041817992 \\
\hline & 38 & Juvenile hormone III & 0.04439326 \\
\hline & 39 & Ricinoleic acid & 0.044670352 \\
\hline & 40 & Methdilazine & 0.046513563 \\
\hline
\end{tabular}


Table 6: Information of metabolic pathways.

\begin{tabular}{|c|c|c|c|c|c|c|c|c|c|}
\hline No. & Pathway Name & Total & Hits & Expected & Raw $p$ & $-\log (p)$ & Holm adjust & FDR & Impact \\
\hline 1 & Phenylalanine metabolism & 45 & 3 & 0.74782 & 0.03727 & 3.2896 & 1 & 0.99387 & 0.29159 \\
\hline 2 & Alanine, aspartate and glutamate metabolism & 24 & 1 & 0.39884 & 0.33245 & 1.1013 & 1 & 1 & 0.17664 \\
\hline 3 & Valine, leucine and isoleucine biosynthesis & 27 & 4 & 0.44869 & 0.0008717 & 7.0451 & 0.06974 & 0.06974 & 0.14573 \\
\hline 4 & D-Glutamine and D-glutamate metabolism & 11 & 1 & 0.1828 & 0.16867 & 1.7798 & 1 & 1 & 0.1123 \\
\hline 5 & Aminoacyl-tRNA biosynthesis & 75 & 4 & 1.2464 & 0.034037 & 3.3803 & 1 & 0.99387 & 0.05634 \\
\hline 6 & Drug metabolism - cytochrome P450 & 86 & 1 & 1.4292 & 0.76949 & 0.26203 & 1 & 1 & 0.04839 \\
\hline 7 & Nicotinate and nicotinamide metabolism & 44 & 1 & 0.7312 & 0.52482 & 0.64469 & 1 & 1 & 0.04454 \\
\hline 8 & Arginine and proline metabolism & 77 & 1 & 1.2796 & 0.73054 & 0.31397 & 1 & 1 & 0.03582 \\
\hline 9 & Fructose and mannose metabolism & 48 & 1 & 0.79767 & 0.55621 & 0.58661 & 1 & 1 & 0.03029 \\
\hline 10 & Arachidonic acid metabolism & 62 & 1 & 1.0303 & 0.65093 & 0.42935 & 1 & 1 & 0.0255 \\
\hline 11 & Valine, leucine and isoleucine degradation & 40 & 2 & 0.66473 & 0.14164 & 1.9545 & 1 & 1 & 0.02232 \\
\hline 12 & Tryptophan metabolism & 79 & 1 & 1.3128 & 0.73972 & 0.30149 & 1 & 1 & 0.01143 \\
\hline 13 & Glutathione metabolism & 38 & 1 & 0.63149 & 0.47365 & 0.74729 & 1 & 1 & 0.01095 \\
\hline 14 & Primary bile acid biosynthesis & 47 & 1 & 0.78106 & 0.54856 & 0.60046 & 1 & 1 & 0.00992 \\
\hline 15 & Sphingolipid metabolism & 25 & 1 & 0.41545 & 0.34365 & 1.0681 & 1 & 1 & 0.00191 \\
\hline 16 & Phenylalanine, tyrosine and tryptophan biosynthesis & 27 & 1 & 0.44869 & 0.36552 & 1.0064 & 1 & 1 & 0.00062 \\
\hline 17 & Histidine metabolism & 44 & 1 & 0.7312 & 0.52482 & 0.64469 & 1 & 1 & 0.00051 \\
\hline 18 & Nitrogen metabolism & 39 & 2 & 0.64811 & 0.13593 & 1.9956 & 1 & 1 & 0 \\
\hline 19 & Selenoamino acid metabolism & 22 & 1 & 0.3656 & 0.30948 & 1.1729 & 1 & 1 & 0 \\
\hline 20 & alpha-Linolenic acid metabolism & 29 & 1 & 0.48193 & 0.38667 & 0.95019 & 1 & 1 & 0 \\
\hline 21 & Pyruvate metabolism & 32 & 1 & 0.53178 & 0.41711 & 0.8744 & 1 & 1 & 0 \\
\hline 22 & Butanoate metabolism & 40 & 1 & 0.66473 & 0.49128 & 0.71075 & 1 & 1 & 0 \\
\hline 23 & Porphyrin and chlorophyll metabolism & 104 & 1 & 1.7283 & 0.83162 & 0.18438 & 1 & 1 & 0 \\
\hline
\end{tabular}


Table 7 List of biomarkers of metabolism for $\mathrm{MCl}$ intervened by medium dose of SCPE.

\begin{tabular}{|c|c|c|c|}
\hline Mode & No. & Metabolite & P-value \\
\hline \multirow[t]{26}{*}{ pos } & 1 & Hexadecanedioic acid & 0.000364007 \\
\hline & 2 & Eicosadienoic acid & 0.002506244 \\
\hline & 3 & L-Isoleucine & 0.002960788 \\
\hline & 4 & L-Carnitine & 0.002974809 \\
\hline & 5 & Leukotriene D4 & 0.003270639 \\
\hline & 6 & 4-Hydroxyifosfamide & 0.00355378 \\
\hline & 7 & 11Z-Eicosenoic acid & 0.005266836 \\
\hline & 8 & Angiotensin & 0.007228939 \\
\hline & 9 & trans-Cinnamic acid & 0.007688179 \\
\hline & 10 & Toluene & 0.013619825 \\
\hline & 11 & Methoxamine & 0.015654557 \\
\hline & 12 & D-Phenylalanine & 0.019709825 \\
\hline & 13 & Episterol & 0.019730763 \\
\hline & 14 & Isoproterenol & 0.020552676 \\
\hline & 15 & 4-Hydroxycinnamic acid & 0.021222921 \\
\hline & 16 & 2-Arachidonylglycerol & 0.023795703 \\
\hline & 17 & L-Cystathionine & 0.025987653 \\
\hline & 18 & LysoSM(d18:1) & 0.026740733 \\
\hline & 19 & Isopropylmaleate & 0.026761377 \\
\hline & 20 & o-Cresol & 0.028705104 \\
\hline & 21 & Coniferyl aldehyde & 0.029970338 \\
\hline & 22 & $(3 Z, 6 Z)-3,6-$ Nonadienal & 0.030424965 \\
\hline & 23 & L-Glutamic acid & 0.034601098 \\
\hline & 24 & Boldione & 0.037633349 \\
\hline & 25 & Alpha-Tocotrienol & 0.043909143 \\
\hline & 26 & Taurochenodesoxycholic acid & 0.046249079 \\
\hline \multirow[t]{22}{*}{ neg } & 27 & L-Phenylalanine & 0.000411359 \\
\hline & 28 & 16-Hydroxy hexadecanoic acid & 0.002608362 \\
\hline & 29 & Ricinoleic acid & 0.002881789 \\
\hline & 30 & Gibberellin A15 open lactone & 0.004733181 \\
\hline & 31 & 5-Hydroxyindoleacetate & 0.00737662 \\
\hline & 32 & N1-Acetylspermine & 0.008810405 \\
\hline & 33 & Methdilazine & 0.009052052 \\
\hline & 34 & L-Leucine & 0.009499488 \\
\hline & 35 & beta-Tyrosine & 0.017598872 \\
\hline & 36 & Panaxytriol & 0.018113519 \\
\hline & 37 & Triamcinolone diacetate & 0.018457432 \\
\hline & 38 & Benzocaine & 0.022313529 \\
\hline & 39 & L-Norleucine & 0.022543089 \\
\hline & 40 & L-Rhamnulose & 0.023579355 \\
\hline & 41 & 4-Hydroxy-4-(3-pyridyl)-butanoic acid & 0.025276493 \\
\hline & 42 & N-Acetyl-L-histidine & 0.026863662 \\
\hline & 43 & Juvenile hormone III & 0.031969851 \\
\hline & 44 & gamma-Glutamyl-Se-methylselenocysteine & 0.032963008 \\
\hline & 45 & $\left(2^{\prime} \mathrm{E}, 4^{\prime} \mathrm{Z}, 8 \mathrm{E}\right)$-Colneleic acid & 0.039224291 \\
\hline & 46 & (S)-2-(Hydroxymethyl)glutarate & 0.04129192 \\
\hline & 47 & Paraquat dichloride & 0.043638928 \\
\hline & 48 & Telmisartan & 0.045101423 \\
\hline
\end{tabular}


Table 8: Information of metabolic pathways.

\begin{tabular}{|c|c|c|c|c|c|c|c|c|c|}
\hline No. & Pathway Name & Total & Hits & Expected & Raw $\mathbf{p}$ & $-\log (p)$ & Holm adjust & FDR & Impact \\
\hline 1 & Phenylalanine metabolism & 45 & 4 & 0.85999 & 0.009816 & 4.6238 & 0.78527 & 0.55454 & 0.30352 \\
\hline 2 & Alanine, aspartate and glutamate metabolism & 24 & 1 & 0.45866 & 0.37208 & 0.98863 & 1 & 1 & 0.17664 \\
\hline 3 & Cysteine and methionine metabolism & 56 & 1 & 1.0702 & 0.66487 & 0.40817 & 1 & 1 & 0.13277 \\
\hline 4 & D-Glutamine and D-glutamate metabolism & 11 & 1 & 0.21022 & 0.1916 & 1.6523 & 1 & 1 & 0.1123 \\
\hline 5 & Valine, leucine and isoleucine biosynthesis & 27 & 3 & 0.516 & 0.013864 & 4.2785 & 1 & 0.55454 & 0.08214 \\
\hline 6 & Aminoacyl-tRNA biosynthesis & 75 & 4 & 1.4333 & 0.052886 & 2.9396 & 1 & 1 & 0.05634 \\
\hline 7 & Drug metabolism - cytochrome P450 & 86 & 1 & 1.6435 & 0.81544 & 0.20403 & 1 & 1 & 0.04839 \\
\hline 8 & Nicotinate and nicotinamide metabolism & 44 & 1 & 0.84088 & 0.57547 & 0.55257 & 1 & 1 & 0.04454 \\
\hline 9 & Arginine and proline metabolism & 77 & 1 & 1.4715 & 0.77909 & 0.24963 & 1 & 1 & 0.03582 \\
\hline 10 & Ubiquinone and other terpenoid-quinone biosynthesis & 36 & 1 & 0.68799 & 0.50332 & 0.68654 & 1 & 1 & 0.0337 \\
\hline 11 & Fructose and mannose metabolism & 48 & 1 & 0.91732 & 0.60759 & 0.49825 & 1 & 1 & 0.03029 \\
\hline 12 & Arachidonic acid metabolism & 62 & 1 & 1.1849 & 0.70237 & 0.35329 & 1 & 1 & 0.0255 \\
\hline 13 & Valine, leucine and isoleucine degradation & 40 & 2 & 0.76444 & 0.17688 & 1.7323 & 1 & 1 & 0.02232 \\
\hline 14 & Tryptophan metabolism & 79 & 1 & 1.5098 & 0.78773 & 0.23861 & 1 & 1 & 0.01143 \\
\hline 15 & Glutathione metabolism & 38 & 1 & 0.72622 & 0.52241 & 0.64931 & 1 & 1 & 0.01095 \\
\hline 16 & Primary bile acid biosynthesis & 47 & 1 & 0.89821 & 0.59979 & 0.51117 & 1 & 1 & 0.00992 \\
\hline 17 & Sphingolipid metabolism & 25 & 1 & 0.47777 & 0.38421 & 0.95658 & 1 & 1 & 0.00191 \\
\hline 18 & Phenylalanine, tyrosine and tryptophan biosynthesis & 27 & 1 & 0.516 & 0.40776 & 0.89706 & 1 & 1 & 0.00062 \\
\hline 19 & Histidine metabolism & 44 & 1 & 0.84088 & 0.57547 & 0.55257 & 1 & 1 & 0.00051 \\
\hline 20 & Nitrogen metabolism & 39 & 3 & 0.74533 & 0.036948 & 3.2983 & 1 & 0.98527 & 0 \\
\hline 21 & Selenoamino acid metabolism & 22 & 1 & 0.42044 & 0.34714 & 1.058 & 1 & 1 & 0 \\
\hline 22 & alpha-Linolenic acid metabolism & 29 & 1 & 0.55422 & 0.43044 & 0.84294 & 1 & 1 & 0 \\
\hline 23 & Butanoate metabolism & 40 & 1 & 0.76444 & 0.54078 & 0.61475 & 1 & 1 & 0 \\
\hline 24 & Glycine, serine and threonine metabolism & 48 & 1 & 0.91732 & 0.60759 & 0.49825 & 1 & 1 & 0 \\
\hline 25 & Tyrosine metabolism & 76 & 1 & 1.4524 & 0.77464 & 0.25536 & 1 & 1 & 0 \\
\hline 26 & Porphyrin and chlorophyll metabolism & 104 & 1 & 1.9875 & 0.87145 & 0.1376 & 1 & 1 & 0 \\
\hline
\end{tabular}


Table 9 List of biomarkers of metabolism for $\mathrm{MCl}$ intervened by high dose of SCPE.

\begin{tabular}{|c|c|c|c|}
\hline Mode & No. & Metabolite & P-value \\
\hline \multirow[t]{29}{*}{ pos } & 1 & Hexadecanedioic acid & 0.000229493 \\
\hline & 2 & L-Carnitine & 0.000281269 \\
\hline & 3 & trans-Cinnamic acid & 0.000647719 \\
\hline & 4 & Piperidine & 0.001290075 \\
\hline & 5 & 1-Phenyl-1,2-propanedione & 0.001360602 \\
\hline & 6 & L-Isoleucine & 0.001798272 \\
\hline & 7 & Leukotriene D4 & 0.0019856 \\
\hline & 8 & o-Cresol & 0.002559176 \\
\hline & 9 & 4-Hydroxycinnamic acid & 0.002625843 \\
\hline & 10 & Isoproterenol & 0.004984994 \\
\hline & 11 & LysoSM(d18:1) & 0.005815594 \\
\hline & 12 & 11Z-Eicosenoic acid & 0.006075564 \\
\hline & 13 & $(3 \mathrm{Z}, 6 \mathrm{Z})-3,6$-Nonadienal & 0.006386328 \\
\hline & 14 & Alpha-Tocotrienol & 0.006882449 \\
\hline & 15 & Mannitol 1-phosphate & 0.009552884 \\
\hline & 16 & D-Phenylalanine & 0.012434668 \\
\hline & 17 & Angiotensin & 0.013908496 \\
\hline & 18 & L-Cystathionine & 0.014867897 \\
\hline & 19 & 4-Hydroxyifosfamide & 0.017142243 \\
\hline & 20 & Methoxamine & 0.022526855 \\
\hline & 21 & 2-Arachidonylglycerol & 0.023020948 \\
\hline & 22 & Toluene & 0.024859699 \\
\hline & 23 & Episterol & 0.033378326 \\
\hline & 24 & Taurochenodesoxycholic acid & 0.034556685 \\
\hline & 25 & Topotecan & 0.035930445 \\
\hline & 26 & Boldione & 0.036067574 \\
\hline & 27 & L-Glutamic acid & 0.036760782 \\
\hline & 28 & 2-Aminoacrylic acid & 0.044543712 \\
\hline & 29 & Coniferyl aldehyde & 0.048985708 \\
\hline \multirow[t]{25}{*}{ neg } & 30 & L-Phenylalanine & 0.001067238 \\
\hline & 31 & beta-Tyrosine & 0.002178563 \\
\hline & 32 & N1-Acetylspermine & 0.003093681 \\
\hline & 33 & L-Norleucine & 0.00402759 \\
\hline & 34 & 2-Isopropylmalic acid & 0.005715303 \\
\hline & 35 & Methdilazine & 0.006166321 \\
\hline & 36 & Gibberellin A15 open lactone & 0.006629754 \\
\hline & 37 & (S)-2-(Hydroxymethyl)glutarate & 0.009445376 \\
\hline & 38 & 5-Hydroxyindoleacetate & 0.010836668 \\
\hline & 39 & Benzocaine & 0.018071091 \\
\hline & 40 & Ricinoleic acid & 0.025977163 \\
\hline & 41 & $(2 ' E, 4 ' Z, 8 E)$-Colneleic acid & 0.026284967 \\
\hline & 42 & Phenoxybenzamine & 0.026475414 \\
\hline & 43 & $\mathrm{~N}$-Acetyl-L-histidine & 0.027232436 \\
\hline & 44 & Panaxytriol & 0.027730829 \\
\hline & 45 & 3-Hydroxy-4-hydroxymethyl-2-methylpyridine-5-carboxylate & 0.029693081 \\
\hline & 46 & Juvenile hormone III & 0.036518942 \\
\hline & 47 & 16-Hydroxy hexadecanoic acid & 0.037668296 \\
\hline & 48 & L-Leucine & 0.038551263 \\
\hline & 49 & Triamcinolone diacetate & 0.03924337 \\
\hline & 50 & L-Rhamnulose & 0.041703342 \\
\hline & 51 & GDP-glucose & 0.042371144 \\
\hline & 52 & gamma-Glutamyl-Se-methylselenocysteine & 0.04400059 \\
\hline & 53 & 4-Hydroxy-4-(3-pyridyl)-butanoic acid & 0.044712702 \\
\hline & 54 & Telmisartan & 0.047739669 \\
\hline
\end{tabular}


Table 10: Information of metabolic pathways

\begin{tabular}{|c|c|c|c|c|c|c|c|c|c|}
\hline No. & Pathway Name & Total & Hits & Expected & Raw $p$ & $-\log (p)$ & Holm adjust & FDR & Impact \\
\hline 1 & Phenylalanine metabolism & 45 & 4 & 0.99086 & 0.01602 & 4.134 & 1 & 0.81232 & 0.30352 \\
\hline 2 & Alanine, aspartate and glutamate metabolism & 24 & 1 & 0.52846 & 0.41548 & 0.87832 & 1 & 1 & 0.17664 \\
\hline 3 & Cysteine and methionine metabolism & 56 & 2 & 1.2331 & 0.35111 & 1.0467 & 1 & 1 & 0.13505 \\
\hline 4 & D-Glutamine and D-glutamate metabolism & 11 & 1 & 0.24221 & 0.21763 & 1.5249 & 1 & 1 & 0.1123 \\
\hline 5 & Valine, leucine and isoleucine biosynthesis & 27 & 3 & 0.59452 & 0.02031 & 3.8967 & 1 & 0.81232 & 0.09009 \\
\hline 6 & Aminoacyl-tRNA biosynthesis & 75 & 4 & 1.6514 & 0.08078 & 2.5161 & 1 & 1 & 0.05634 \\
\hline 7 & Drug metabolism - cytochrome P450 & 86 & 1 & 1.8936 & 0.8577 & 0.1535 & 1 & 1 & 0.04839 \\
\hline 8 & Nicotinate and nicotinamide metabolism & 44 & 1 & 0.96884 & 0.62791 & 0.46536 & 1 & 1 & 0.04454 \\
\hline 9 & Fructose and mannose metabolism & 48 & 2 & 1.0569 & 0.28551 & 1.2535 & 1 & 1 & 0.03942 \\
\hline 10 & Arginine and proline metabolism & 77 & 1 & 1.6955 & 0.8249 & 0.1925 & 1 & 1 & 0.03582 \\
\hline 11 & Ubiquinone and other terpenoid-quinone biosynthesis & 36 & 1 & 0.79269 & 0.55403 & 0.59054 & 1 & 1 & 0.0337 \\
\hline 12 & Vitamin B6 metabolism & 32 & 1 & 0.70461 & 0.51187 & 0.66969 & 1 & 1 & 0.02697 \\
\hline 13 & Arachidonic acid metabolism & 62 & 1 & 1.3652 & 0.75302 & 0.28367 & 1 & 1 & 0.0255 \\
\hline 14 & Valine, leucine and isoleucine degradation & 40 & 2 & 0.88076 & 0.21954 & 1.5162 & 1 & 1 & 0.02232 \\
\hline 15 & Tryptophan metabolism & 79 & 1 & 1.7395 & 0.83278 & 0.18299 & 1 & 1 & 0.01143 \\
\hline 16 & Glutathione metabolism & 38 & 1 & 0.83673 & 0.57375 & 0.55557 & 1 & 1 & 0.01095 \\
\hline 17 & Primary bile acid biosynthesis & 47 & 1 & 1.0349 & 0.6524 & 0.4271 & 1 & 1 & 0.00992 \\
\hline 18 & Sphingolipid metabolism & 25 & 1 & 0.55048 & 0.42848 & 0.84751 & 1 & 1 & 0.00191 \\
\hline 19 & Starch and sucrose metabolism & 50 & 1 & 1.101 & 0.67531 & 0.39259 & 1 & 1 & 0.00149 \\
\hline 20 & Phenylalanine, tyrosine and tryptophan biosynthesis & 27 & 1 & 0.59452 & 0.45363 & 0.79046 & 1 & 1 & 0.00062 \\
\hline 21 & Histidine metabolism & 44 & 1 & 0.96884 & 0.62791 & 0.46536 & 1 & 1 & 0.00051 \\
\hline 22 & Nitrogen metabolism & 39 & 3 & 0.85875 & 0.05278 & 2.9416 & 1 & 1 & 0 \\
\hline 23 & Selenoamino acid metabolism & 22 & 1 & 0.48442 & 0.3886 & 0.9452 & 1 & 1 & 0 \\
\hline 24 & alpha-Linolenic acid metabolism & 29 & 1 & 0.63855 & 0.4777 & 0.73877 & 1 & 1 & 0 \\
\hline 25 & Pyruvate metabolism & 32 & 1 & 0.70461 & 0.51187 & 0.66969 & 1 & 1 & 0 \\
\hline 26 & Butanoate metabolism & 40 & 1 & 0.88076 & 0.59261 & 0.52322 & 1 & 1 & 0 \\
\hline 27 & Glycine, serine and threonine metabolism & 48 & 1 & 1.0569 & 0.66021 & 0.4152 & 1 & 1 & 0 \\
\hline 28 & Tyrosine metabolism & 76 & 1 & 1.6735 & 0.82082 & 0.19745 & 1 & 1 & 0 \\
\hline 29 & Amino sugar and nucleotide sugar metabolism & 88 & 1 & 1.9377 & 0.86413 & 0.14603 & 1 & 1 & 0 \\
\hline 30 & Porphyrin and chlorophyll metabolism & 104 & 1 & 2.29 & 0.90625 & 0.098435 & 1 & 1 & 0 \\
\hline
\end{tabular}


Table 11 Information of metabolic markers in different doses of SCPE. $\cap$ stands for intersection.

\begin{tabular}{|c|c|c|c|c|c|}
\hline No. & Metabolite & Existing group & No. & Metabolite & Existing group \\
\hline 1 & Hexadecanedioic acid & $\mathrm{LD} \cap \mathrm{MD} \cap \mathrm{HD}$ & 30 & $\begin{array}{l}\text { gamma-Glutamyl-Se- } \text { methylse- } \\
\text { lenocysteine }\end{array}$ & $\mathrm{LD} \cap \mathrm{MD} \cap \mathrm{HD}$ \\
\hline 2 & L-Isoleucine & $L D \cap M D \cap H D$ & 31 & N-Acetyl-L-histidine & $\mathrm{LD} \cap \mathrm{MD} \cap \mathrm{HD}$ \\
\hline 3 & Leukotriene D4 & $\mathrm{LD} \cap \mathrm{MD} \cap \mathrm{HD}$ & 32 & (S)-2-(Hydroxymethyl) glutarate & $\mathrm{LD} \cap \mathrm{MD} \cap \mathrm{HD}$ \\
\hline 4 & 11Z-Eicosenoic acid & $\mathrm{LD} \cap \mathrm{MD} \cap \mathrm{HD}$ & 33 & $\left(2^{\prime} E, 4^{\prime} Z, 8 E\right)$-Colneleic acid & $L D \cap M D \cap H D$ \\
\hline 5 & D-Phenylalanine & $\mathrm{LD} \cap \mathrm{MD} \cap \mathrm{HD}$ & 34 & Juvenile hormone III & $L D \cap M D \cap H D$ \\
\hline 6 & trans-Cinnamic acid & $\mathrm{LD} \cap \mathrm{MD} \cap \mathrm{HD}$ & 35 & Ricinoleic acid & $\mathrm{LD} \cap \mathrm{MD} \cap \mathrm{HD}$ \\
\hline 7 & Methoxamine & $L D \cap M D \cap H D$ & 36 & Methdilazine & $\mathrm{LD} \cap \mathrm{MD} \cap \mathrm{HD}$ \\
\hline 8 & (3Z,6Z)-3,6-Nonadienal & $\mathrm{LD} \cap \mathrm{MD} \cap \mathrm{HD}$ & 37 & Eicosadienoic acid & $\mathrm{LD} \cap \mathrm{MD}$ \\
\hline 9 & Isoproterenol & $\mathrm{LD} \cap \mathrm{MD} \cap \mathrm{HD}$ & 38 & Isopropylmaleate & $\mathrm{LD} \cap \mathrm{MD}$ \\
\hline 10 & Toluene & $\mathrm{LD} \cap \mathrm{MD} \cap \mathrm{HD}$ & 39 & Paraquat dichloride & $\mathrm{LD} \cap \mathrm{MD}$ \\
\hline 11 & o-Cresol & $\mathrm{LD} \cap \mathrm{MD} \cap \mathrm{HD}$ & 40 & 2-Isopropylmalic acid & $\mathrm{LD} \cap \mathrm{HD}$ \\
\hline 12 & Angiotensin & $\mathrm{LD} \cap \mathrm{MD} \cap \mathrm{HD}$ & 41 & L-Carnitine & $\mathrm{MD} \cap \mathrm{HD}$ \\
\hline 13 & Boldione & $L D \cap M D \cap H D$ & 42 & 4-Hydroxycinnamic acid & $\mathrm{MD} \cap \mathrm{HD}$ \\
\hline 14 & 2-Arachidonylglycerol & $\mathrm{LD} \cap \mathrm{MD} \cap \mathrm{HD}$ & 43 & L-Cystathionine & $\mathrm{MD} \cap \mathrm{HD}$ \\
\hline 15 & Coniferyl aldehyde & $\mathrm{LD} \cap \mathrm{MD} \cap \mathrm{HD}$ & 44 & Alpha-Tocotrienol & $\mathrm{MD} \cap \mathrm{HD}$ \\
\hline 16 & 4-Hydroxyifosfamide & $\mathrm{LD} \cap \mathrm{MD} \cap \mathrm{HD}$ & 45 & beta-Tyrosine & $\mathrm{MD} \cap \mathrm{HD}$ \\
\hline 17 & L-Glutamic acid & $\mathrm{LD} \cap \mathrm{MD} \cap \mathrm{HD}$ & 46 & Triamcinolone diacetate & $\mathrm{MD} \cap \mathrm{HD}$ \\
\hline 18 & LysoSM(d18:1) & $L D \cap M D \cap H D$ & 47 & Benzocaine & $\mathrm{MD} \cap \mathrm{HD}$ \\
\hline 19 & Episterol & $L D \cap M D \cap H D$ & 48 & L-Norleucine & $\mathrm{MD} \cap \mathrm{HD}$ \\
\hline 20 & $\begin{array}{l}\text { Taurochenodesoxycholic } \\
\text { acid }\end{array}$ & $\mathrm{LD} \cap \mathrm{MD} \cap \mathrm{HD}$ & 49 & $\begin{array}{l}\text { 4-Hydroxy-4-(3-pyridyl) -butanoic } \\
\text { acid }\end{array}$ & $\mathrm{MD} \cap \mathrm{HD}$ \\
\hline 21 & N1-Acetylspermine & $\mathrm{LD} \cap \mathrm{MD} \cap \mathrm{HD}$ & 50 & Piperidine & HD \\
\hline 22 & L-Phenylalanine & $\mathrm{LD} \cap \mathrm{MD} \cap \mathrm{HD}$ & 51 & 1-Phenyl-1,2-propanedione & HD \\
\hline 23 & $\begin{array}{l}\text { Gibberellin A15 open lac- } \\
\text { tone }\end{array}$ & $\mathrm{LD} \cap \mathrm{MD} \cap \mathrm{HD}$ & 52 & Mannitol 1-phosphate & $\mathrm{HD}$ \\
\hline 24 & L-Leucine & $\mathrm{LD} \cap \mathrm{MD} \cap \mathrm{HD}$ & 53 & Top & HD \\
\hline 25 & $\begin{array}{l}\text { 16-Hydroxy hexadecanoic } \\
\text { acid }\end{array}$ & $\mathrm{LD} \cap \mathrm{MD} \cap \mathrm{HD}$ & 54 & 2-Aminoacrylic acid & $\mathrm{HD}$ \\
\hline 26 & Telmisartan & $L D \cap M D \cap H D$ & 55 & Phenoxybenzamine & HD \\
\hline 27 & 5-Hydroxyindoleacetate & $\mathrm{LD} \cap \mathrm{MD} \cap \mathrm{HD}$ & 56 & $\begin{array}{l}\text { 3-Hydroxy-4-hydroxymethyl-2- } \\
\text { methylpyridine-5-carboxylate }\end{array}$ & $\mathrm{HD}$ \\
\hline 28 & L-Rhamnulose & $L D \cap M D \cap H D$ & 57 & GDP-glucose & $\mathrm{HD}$ \\
\hline 29 & Panaxytriol & $\mathrm{LD} \cap \mathrm{MD} \cap \mathrm{HD}$ & & & \\
\hline
\end{tabular}


Figures
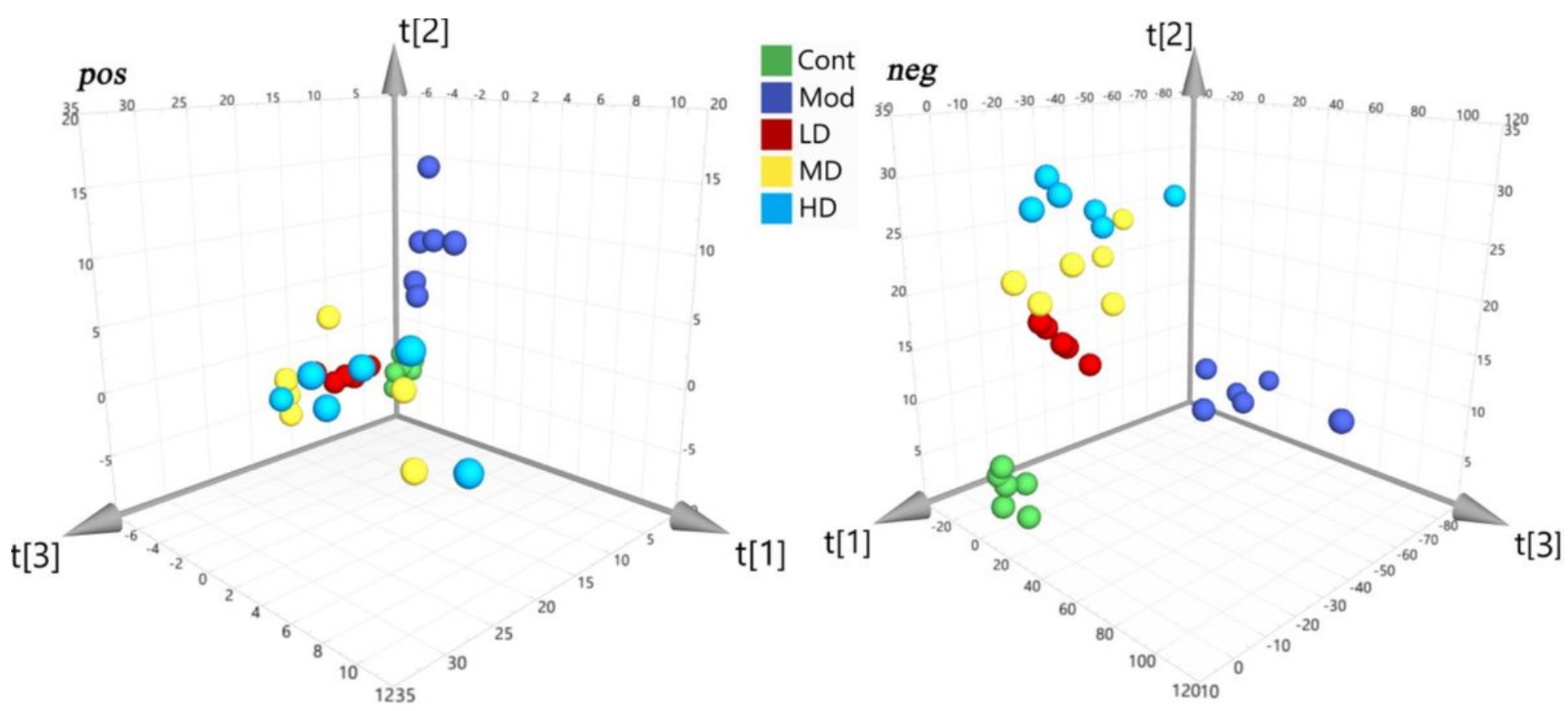

Figure 1

PCA score plots in the mode of positive and negative ions. 

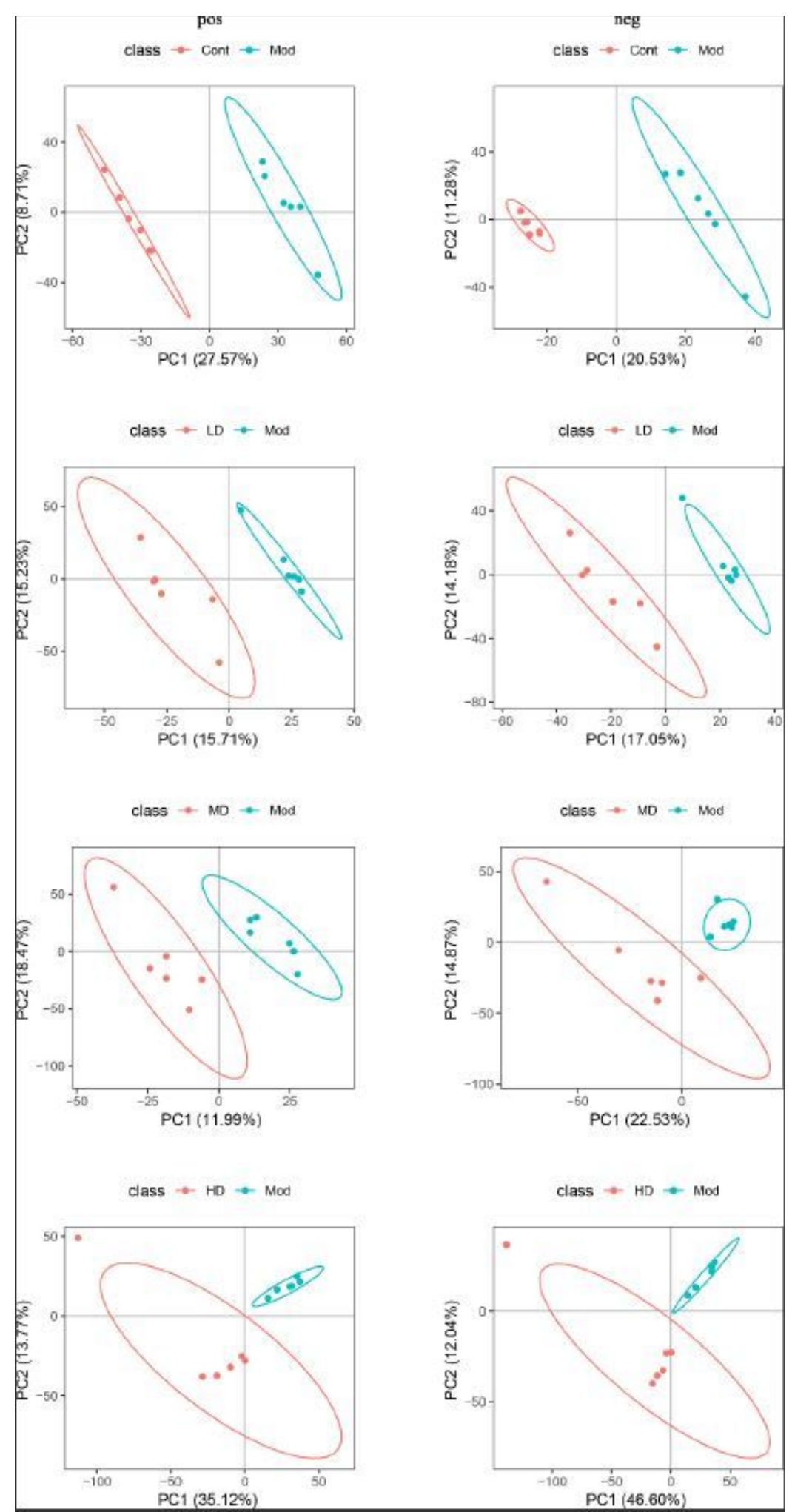

Figure 2

PLS-DA score plots of model analysing. 

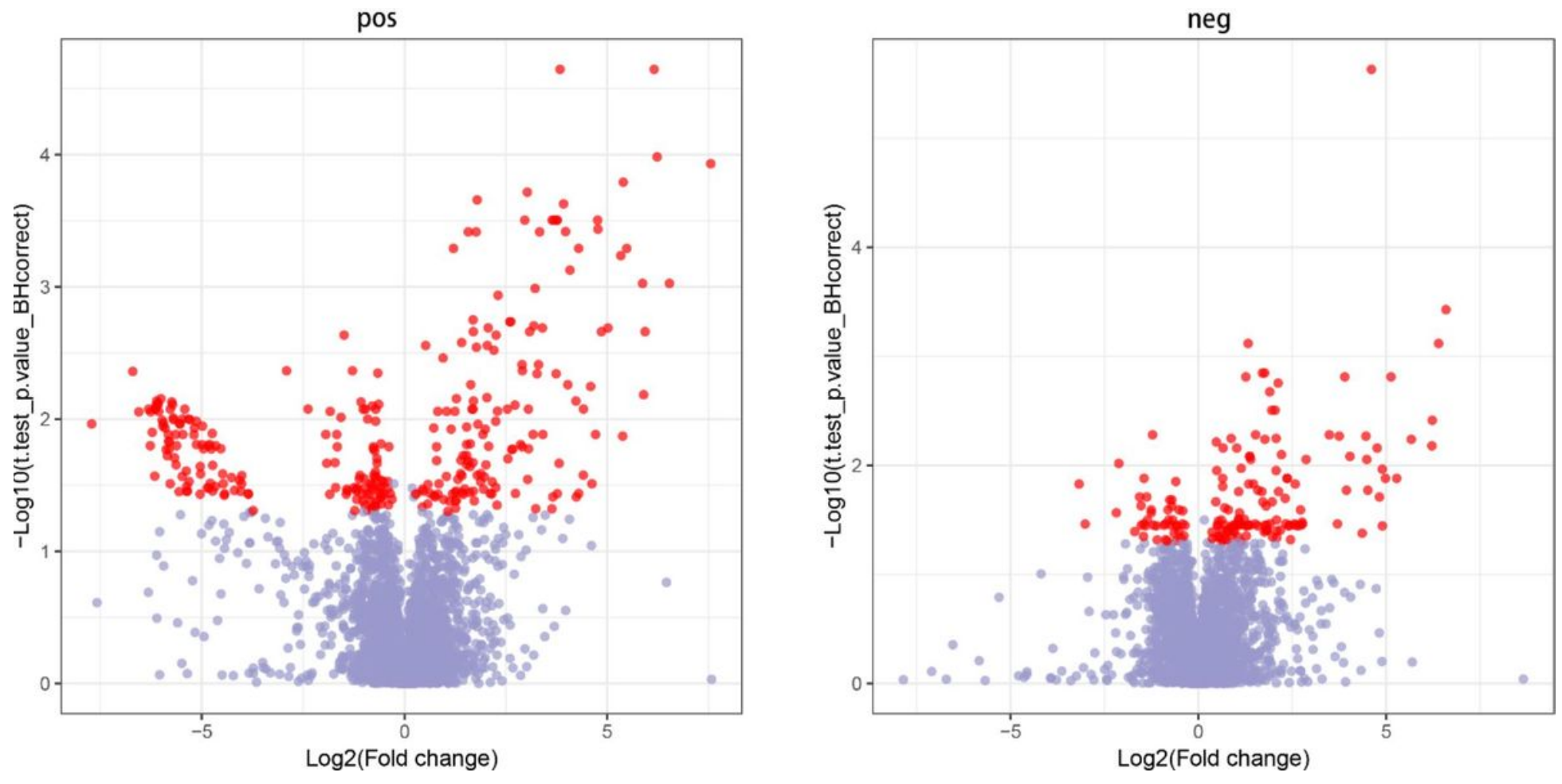

Figure 3

Volcano plot of di囚erential ion. 


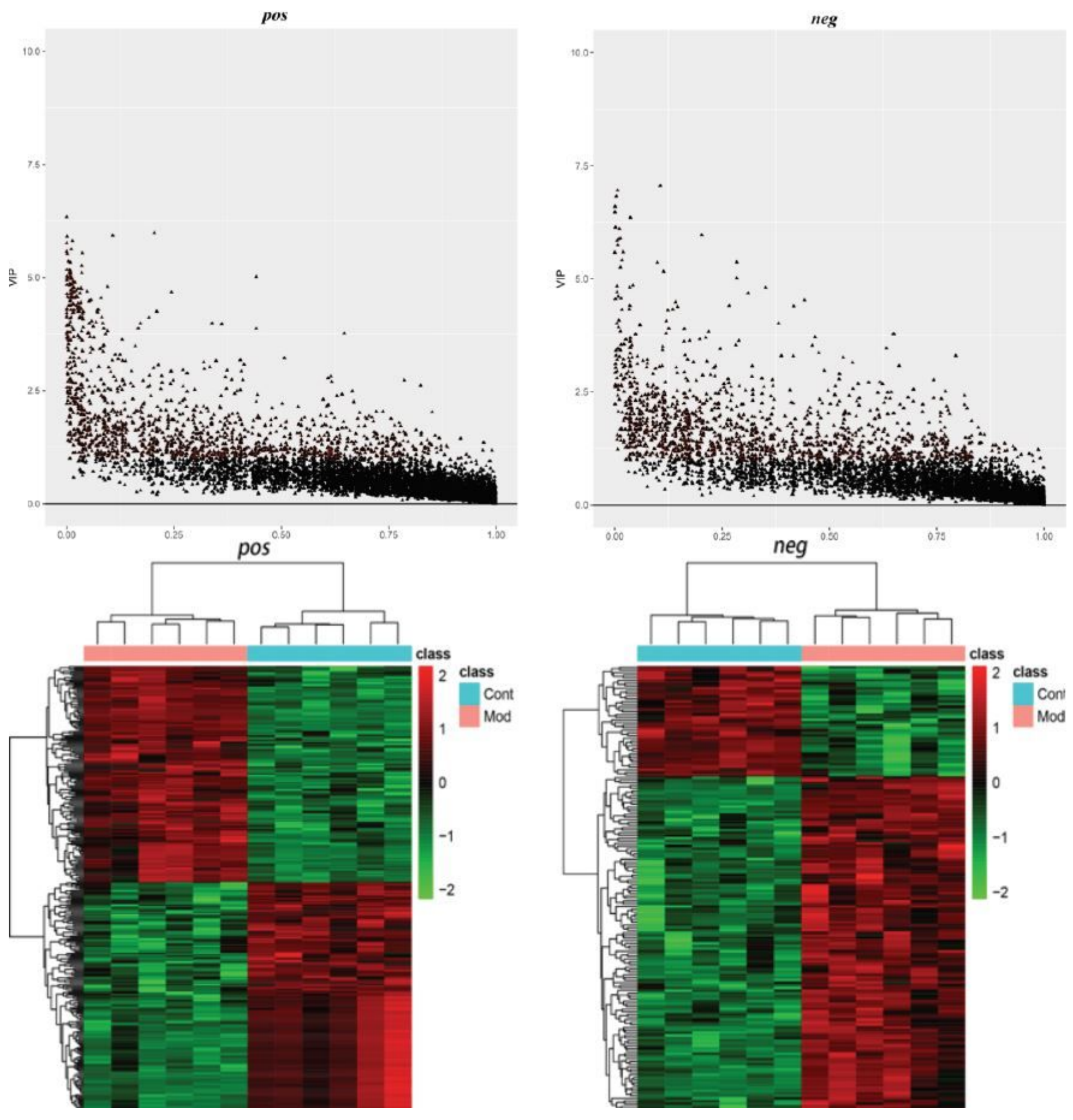

Figure 4

VIP map and Heat map of differential ion clustering analysis. 

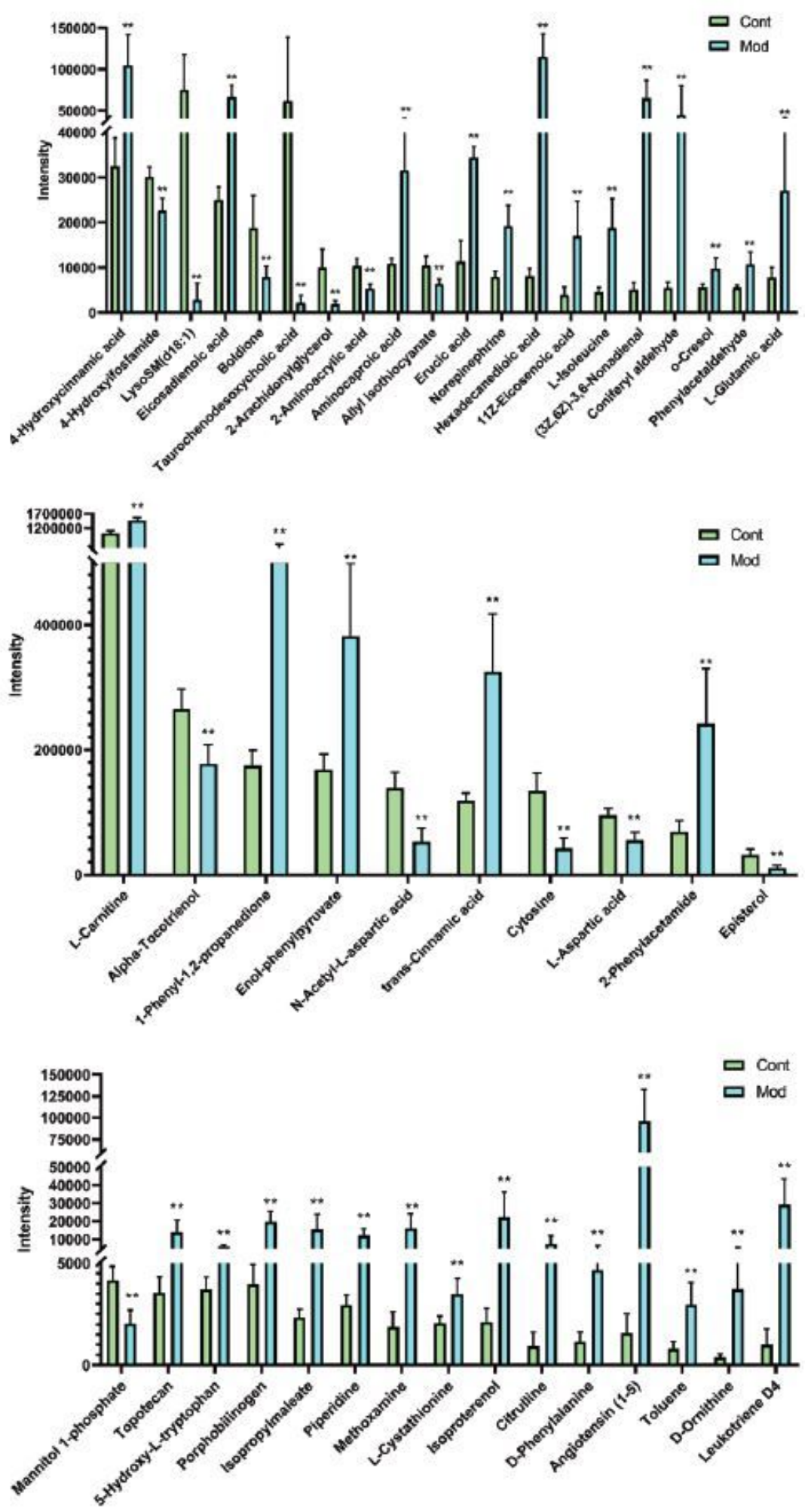

Figure 5

Changes of metabolic markers in the control group and the model group under the positive ion mode. Compared with the blank group, ${ }^{\star * P}$-value $<0.01$. 


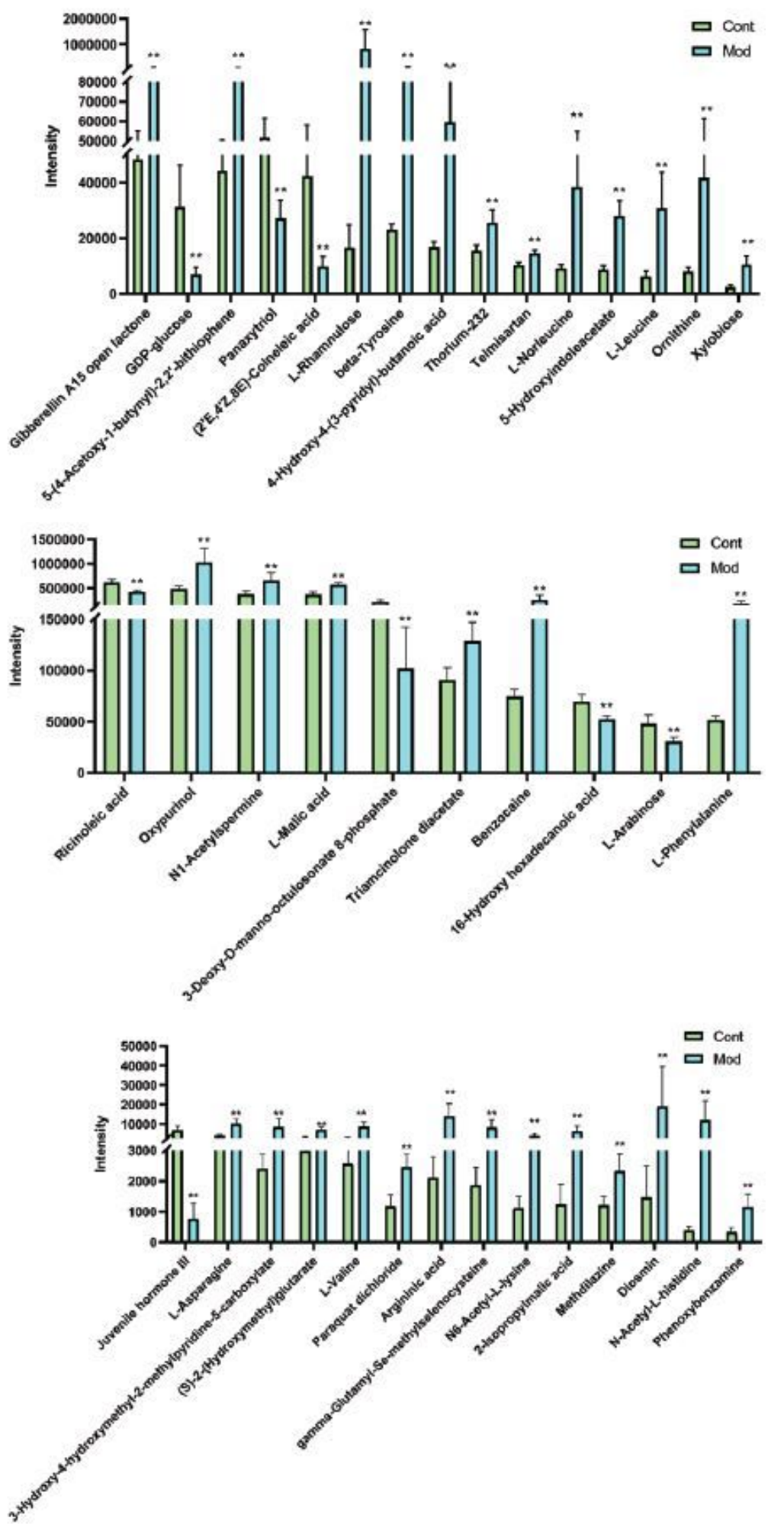

Figure 6

Changes of metabolic markers in the control group and the model group under the negitive ion mode. Compared with the blank group, ${ }^{*} \mathrm{P}$-value $<0.01$. 


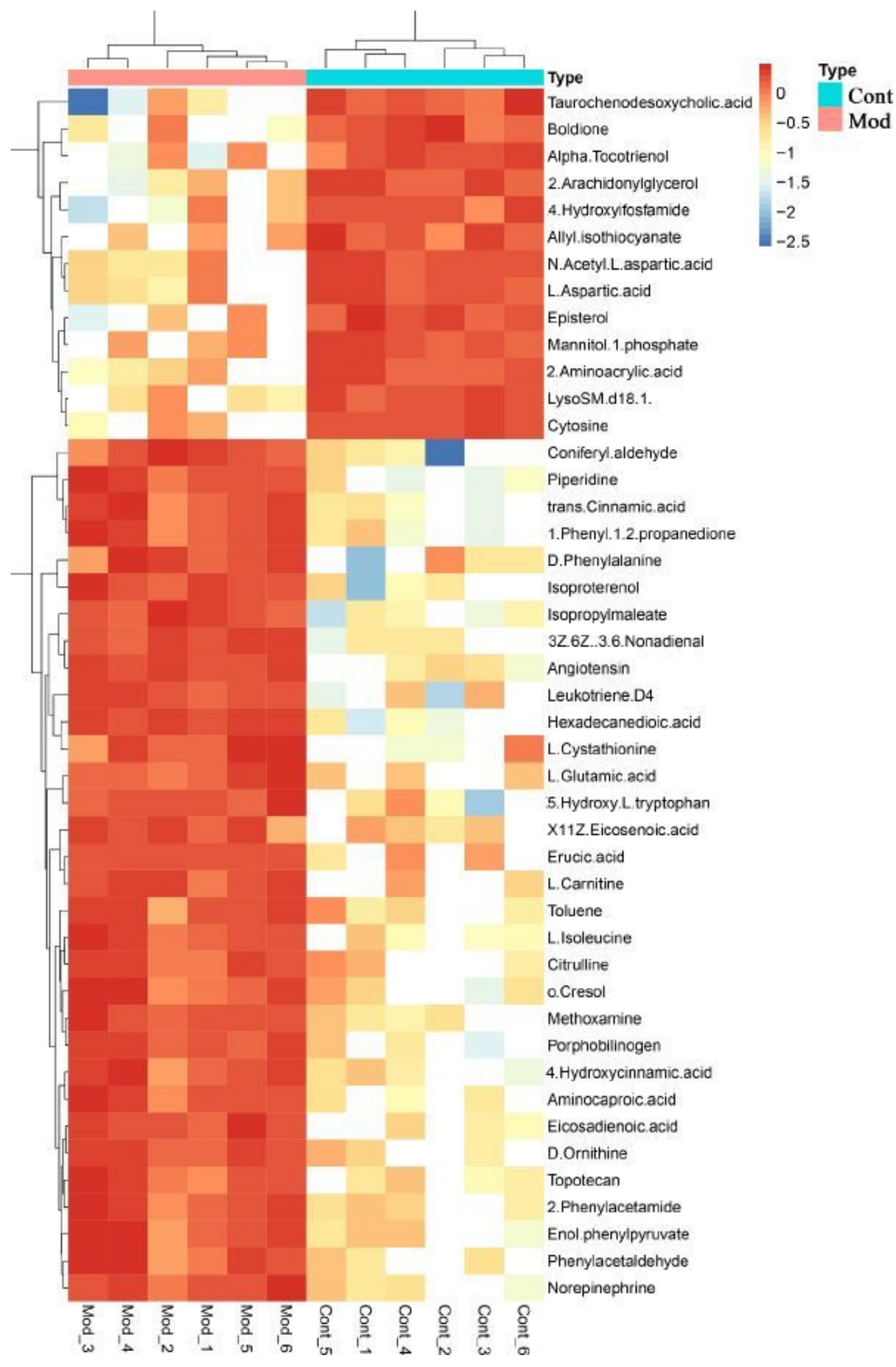

Figure 7

Heat map of differential metabolites cluster analysis under the positive ion mode. 


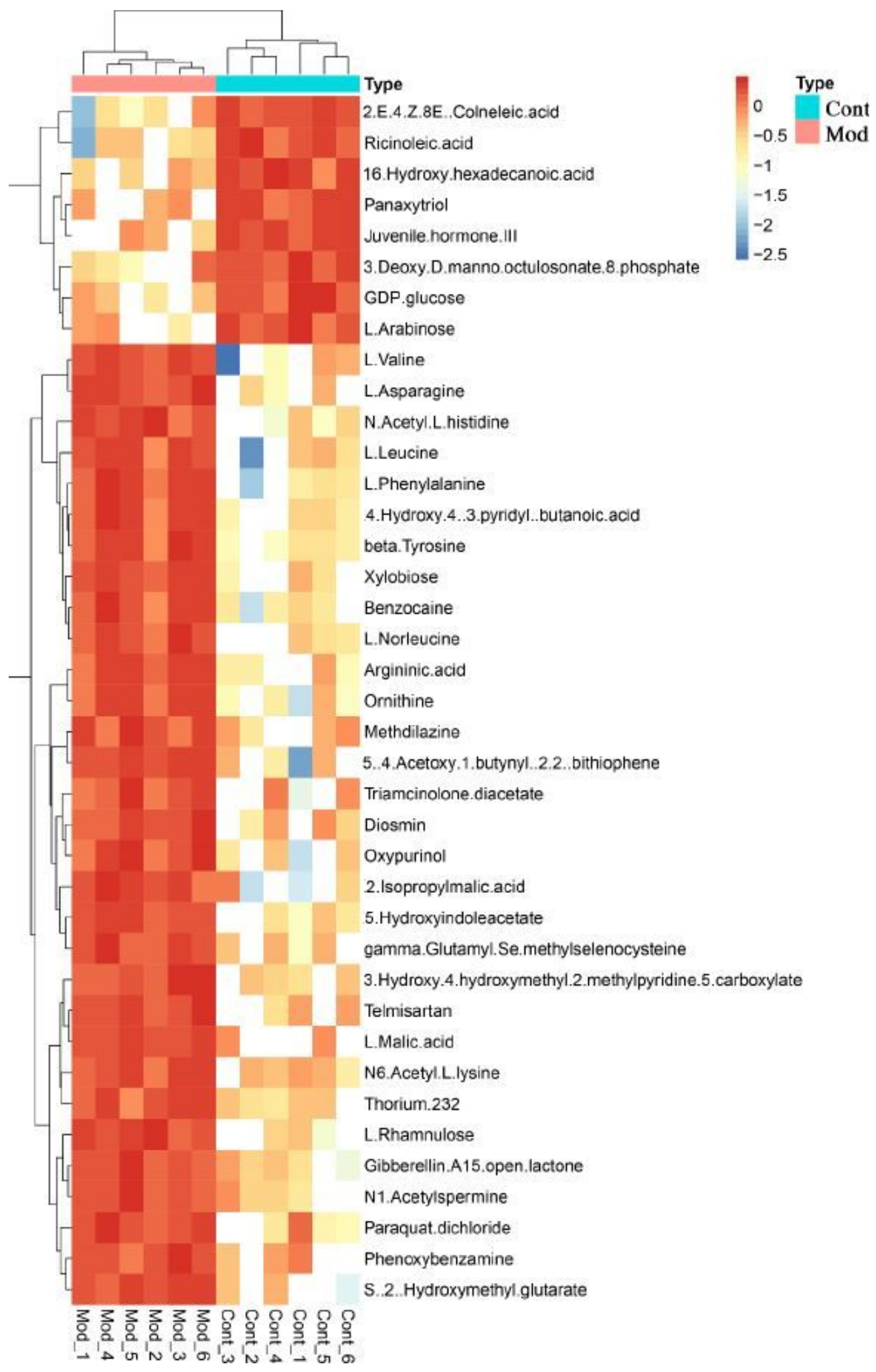

\section{Figure 8}

Heat map of differential metabolites cluster analysis under the negitive ion mode. 


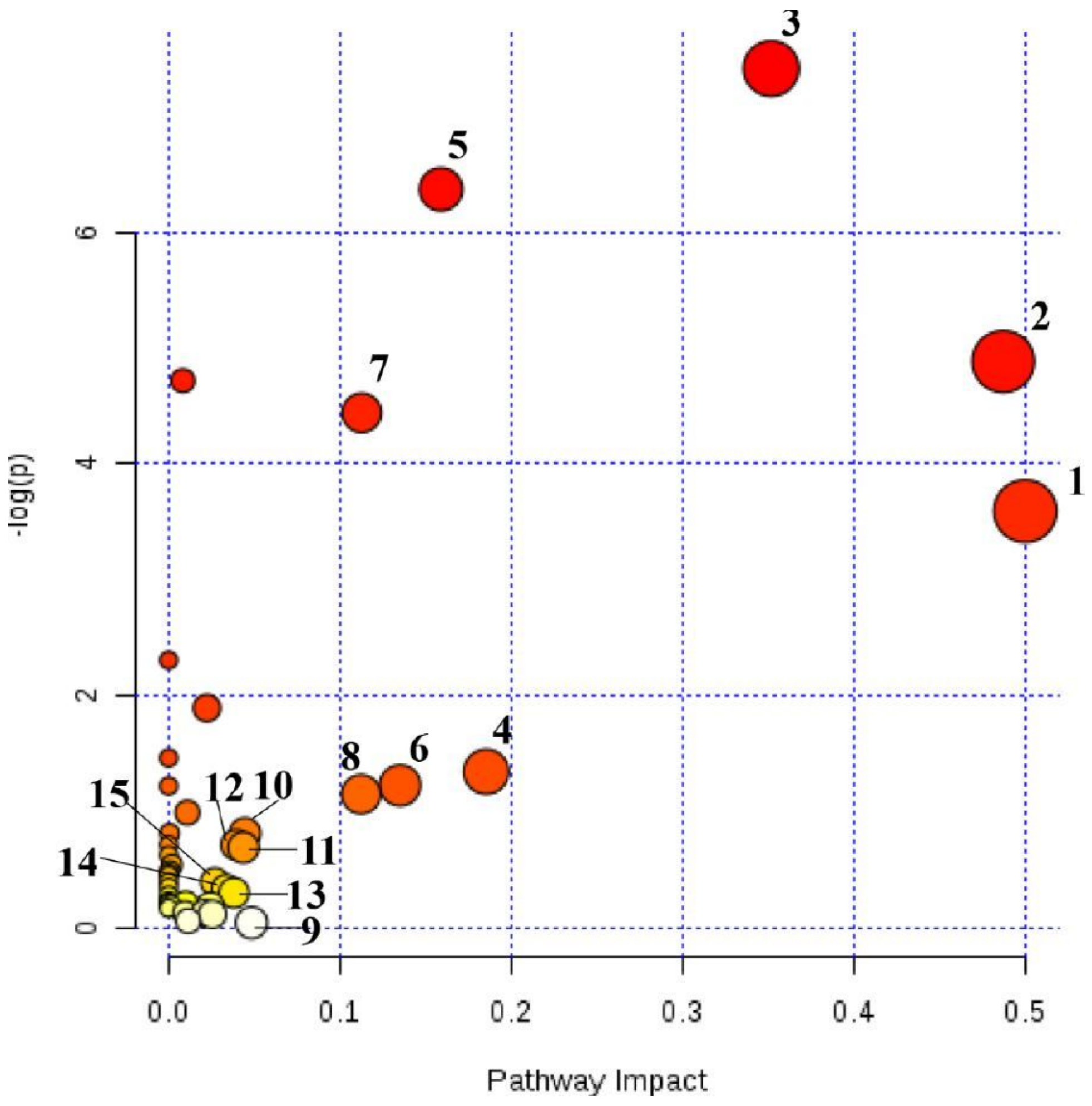

Figure 9

Metabolic pathway analysis of $\mathrm{MCl}$ biomarkers. 
Pentose and glucuronate interconversions -10

Lysine degradation- 0

Ascorbate and aldarate metabolism- 0

Butanoate metabolism- 0

Propanoate metabolism- 0

Lysine biosynthesis -0

alpha-Linolenic acid metabolism- 0

beta-Alanine metabolism-

Selenoamino acid metabolism- 0

Glycine, serine and threonine metabolism- 0

Pyruvate metabolism- 0

Pantothenate and $\mathrm{CoA}$ biosynthesis- 0

Cyanoamino acid metabolism- 0

Amino sugar and nucleotide sugar metabolism- 0.0001

Histidine metabolism- 0.0005

Phenylalanine, tyrosine and tryptophan biosynthesis- 0.0006

Starch and sucrose metabolism- 0.0015

Sphingolipid metabolism- 0.0019

Nitrogen metabolism- 0.0083

Porphyrin and chlorophyll metabolism- ${ }^{0.0095}$

Primary bile acid biosynthesis- 0.0099

Glutathione metabolism 0.0110

Tryptophan metabolism- 0.0114

Pyrimidine metabolism-

Valine, leucine and isoleucine degradation-

Glyoxylate and dicarboxylate metabolism-

Arachidonic acid metabolism $\square_{0.0255}$

Vitamin B6 metabolism- 0.0270

Jbiquinone and other terpenoid-quinone biosynthesis-

Tyrosine metabolism- 0.0381

Fructose and mannose metabolism- 0.0394

Citrate cycle (TCA cycle)

Nicotinate and nicotinamide metabolism- 0.0445

Drug metabolism - cytochrome P450- 0

D-Glutamine and D-glutamate metabolism- 0.1123

Aminoacyl-tRNA biosynthesis 0.1127

Cysteine and methionine metabolism- 0.1351

Valine, leucine and isoleucine biosynthesis 0.1590

Arginine and proline metabolism- 0.1854

Phenylalanine metabolism 0.3518

Alanine, aspartate and glutamate metabolism- 0.4872

D-Arginine and D-ornithine metabolism- 0.5000

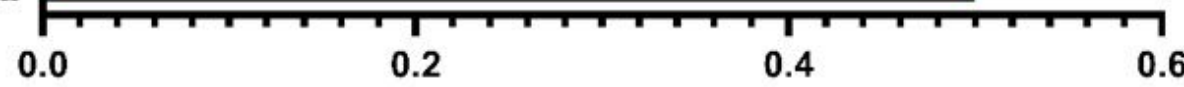

Impact

Figure 10

Metabolic pathway enrichment analysis of $\mathrm{MCl}$ biomarkers. 


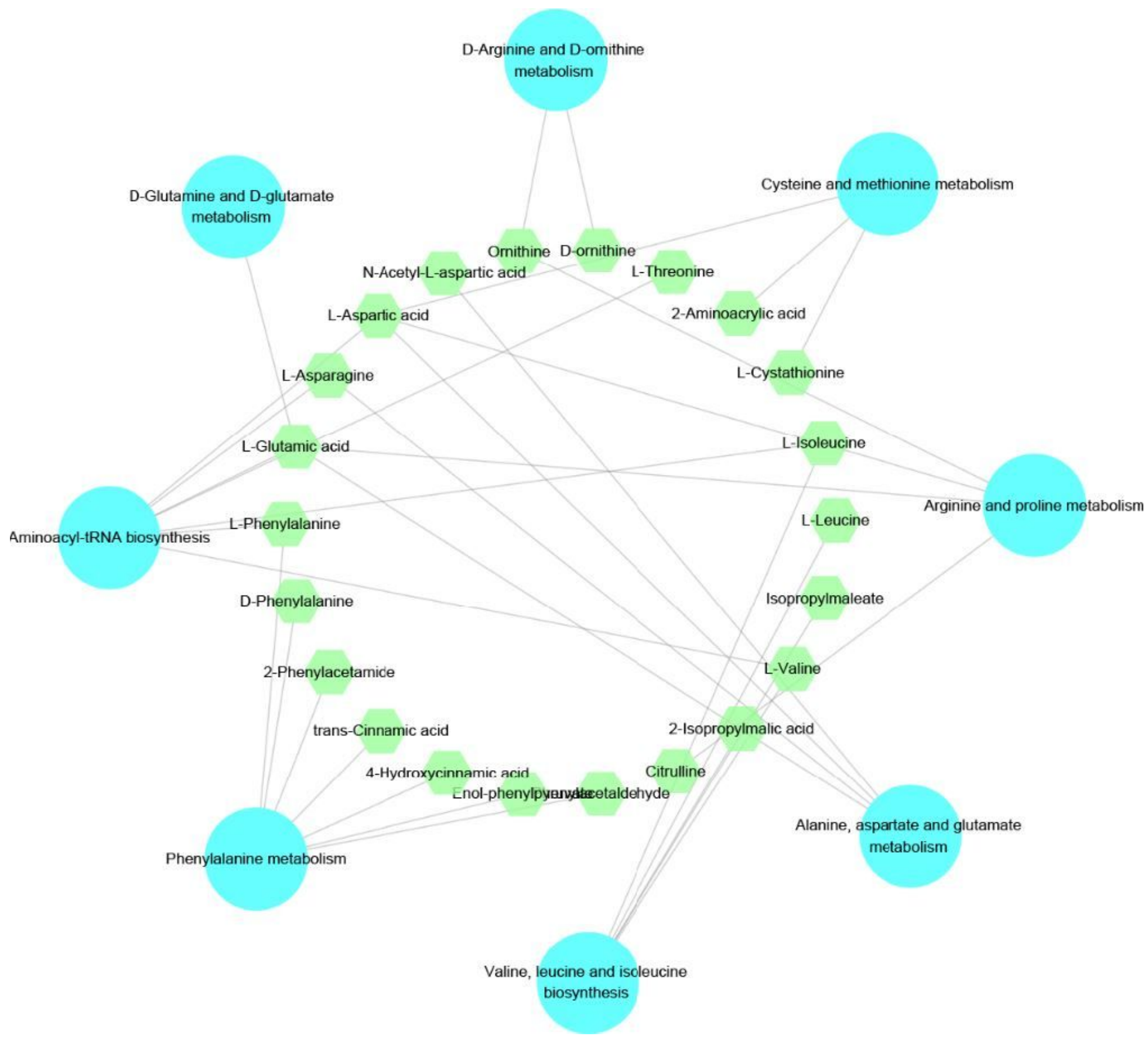

\section{Figure 11}

Metabolic pathway network diagram of $\mathrm{MCl}$ mice. 
A

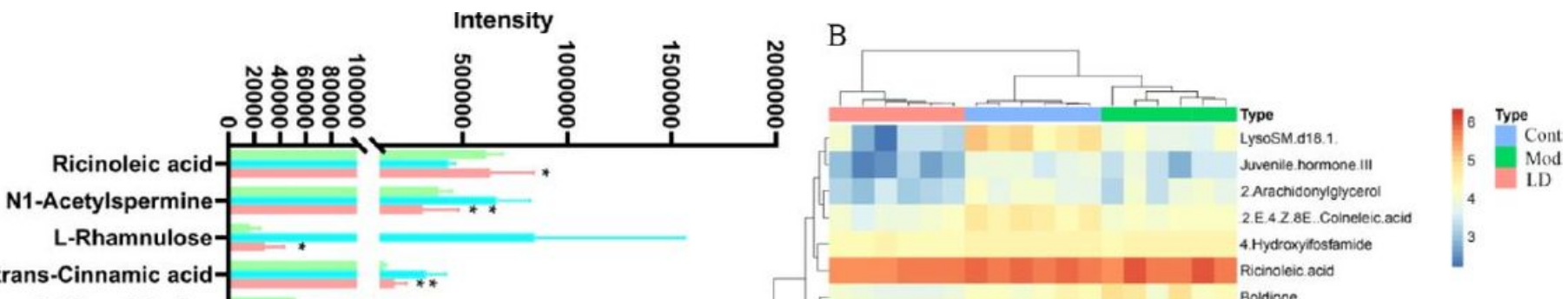

16-Hydroxy hexadecanoic acid-

Gibberellin A15 open lactone-

Hexadecanedioic acid-

Panaxytriol-

Angiotensin

Eicosadienoic acid-

2-Arachidonylglycerol

(3Z,6Z)-3,6-Nonadienal

Taurochenodesoxycholic acid-

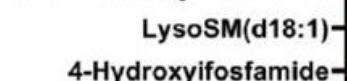

(2'E,4'Z,8E)-Colneleic acid-

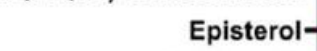

Episterol-

Coniferyl aldehyde-

L-Leucine- *

5-Hydroxyindoleacetate-

L-Glutamic acid-

Telmisartan- *

Leukotriene D4- **

L-Isoleucine- ***

Isoproterenol- *

112-Eicosenoic acid- **

Methoxamine- *

o-Cresol- *

Figure 12

Metabolic biomarkers of $\mathrm{MCl}$ intervented by low dose of SCPE. (A) low dose (LD) SCPE intervention MCI biomarker intensity histogram, compared with the model group (Mod), *P-value $<0.05$; **P-value $<0.01$; (B) biomarker heat map. The color is proportional to the di囚erence in metabolites (red: up-regulated; blue: down-regulated). 


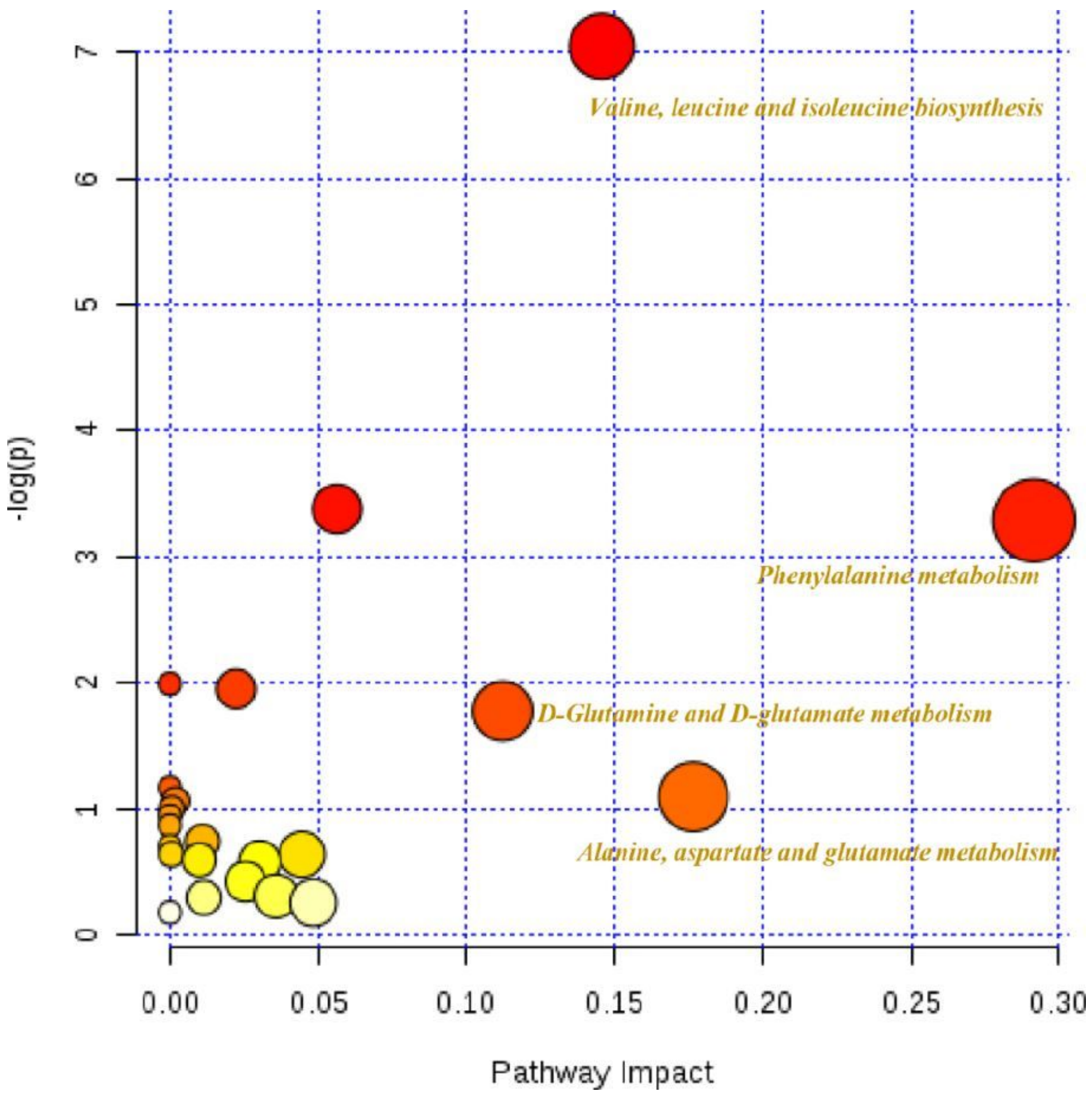

Figure 13

Pathway analysis of biomarkers for $\mathrm{MCl}$ intervention with low dose of SCPE. 


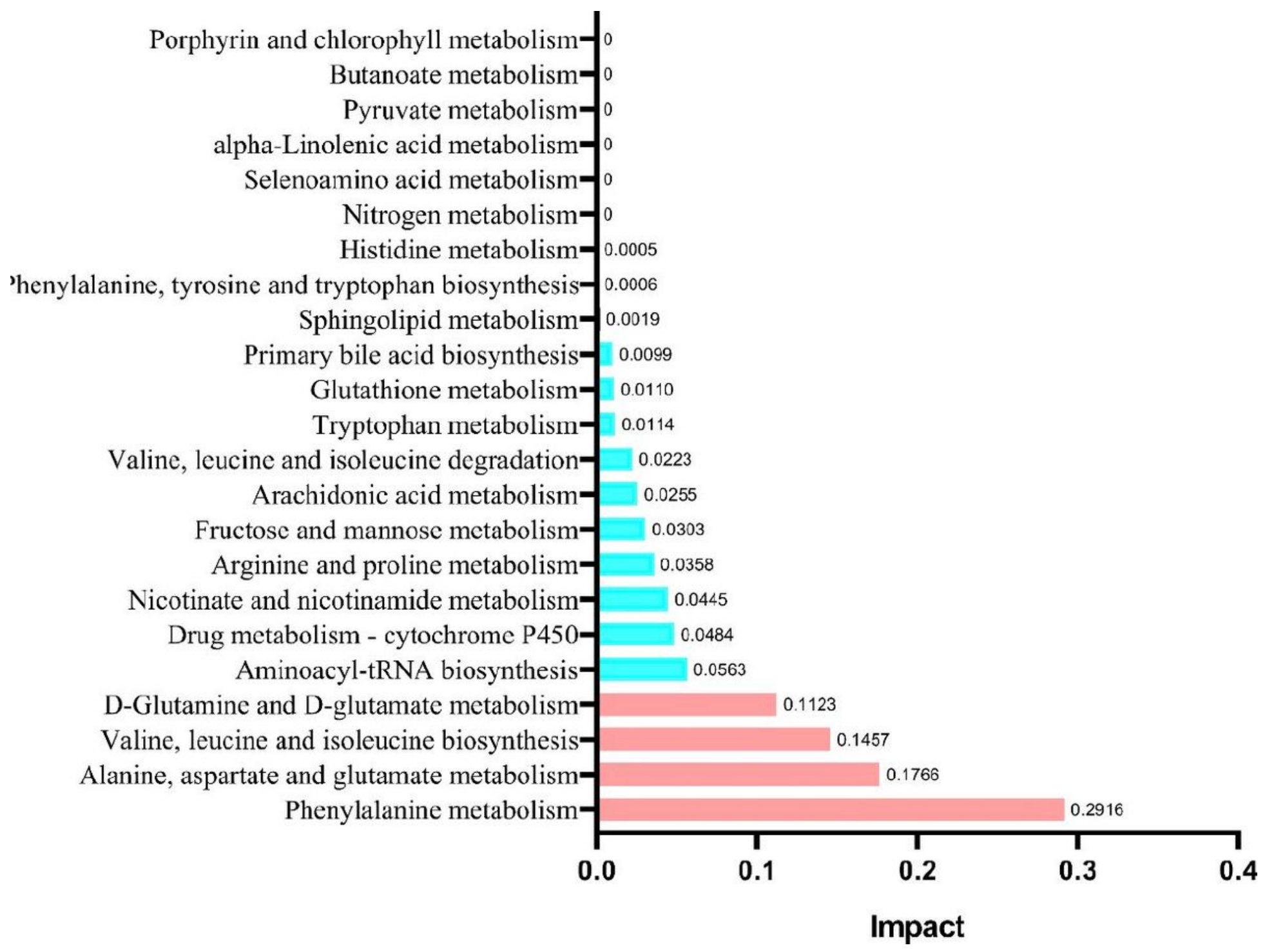

Figure 14

Metabolic pathway enrichment analysis of $\mathrm{MCl}$ biomarkers intervened by SCPE. 


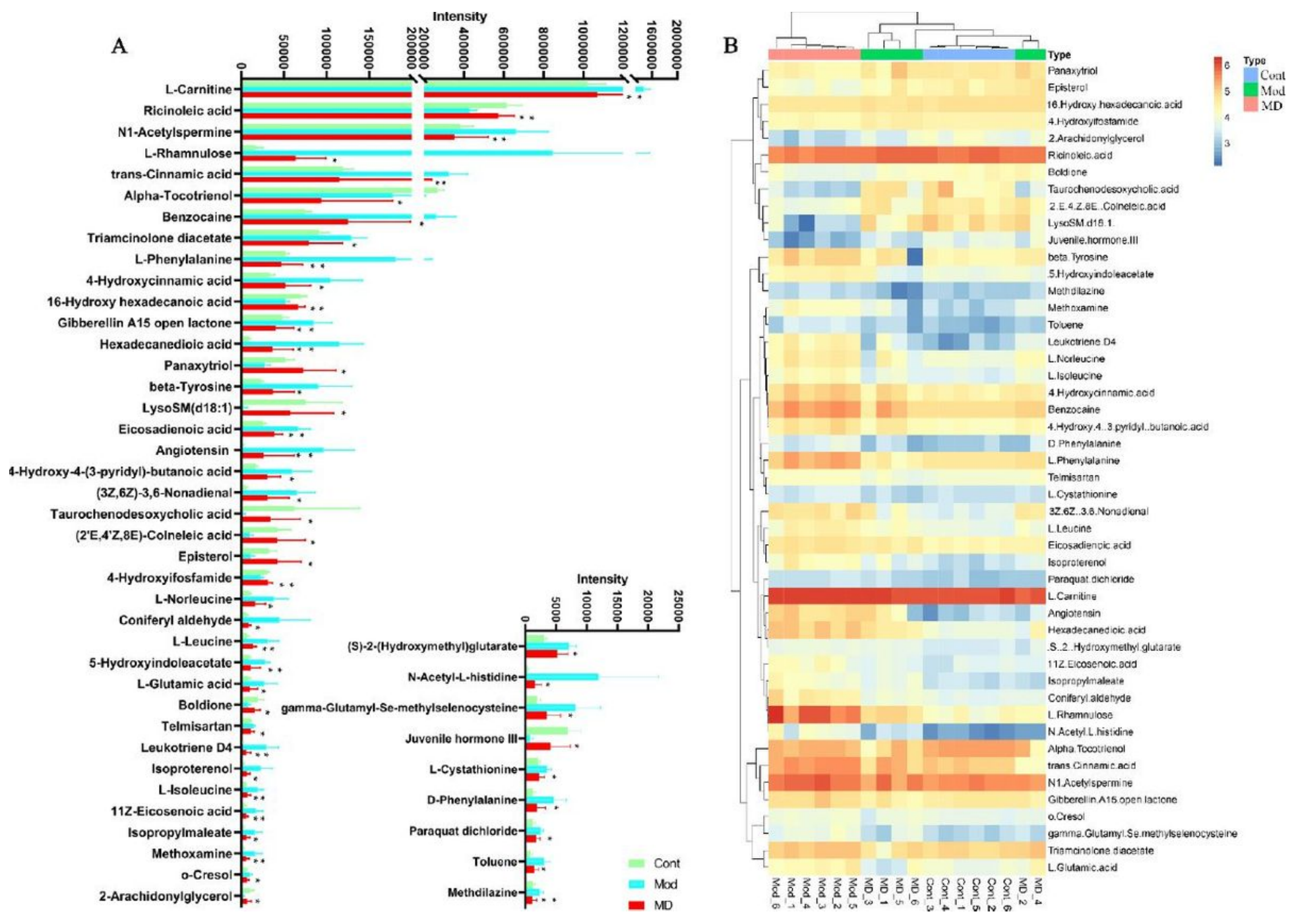

Figure 15

Metabolic biomarkers of $\mathrm{MCl}$ intervented by medium dose of SCPE. (A) Histogram of biomarker intensity of medium dose (MD) intervention in $\mathrm{MCl}$, compared with the model group (Mod) *P-value < 0.05 ; $*$ * Pvalue $<0.01$; (B)Heat map of biomarkers (red: up-regulated; blue: down-regulated). 


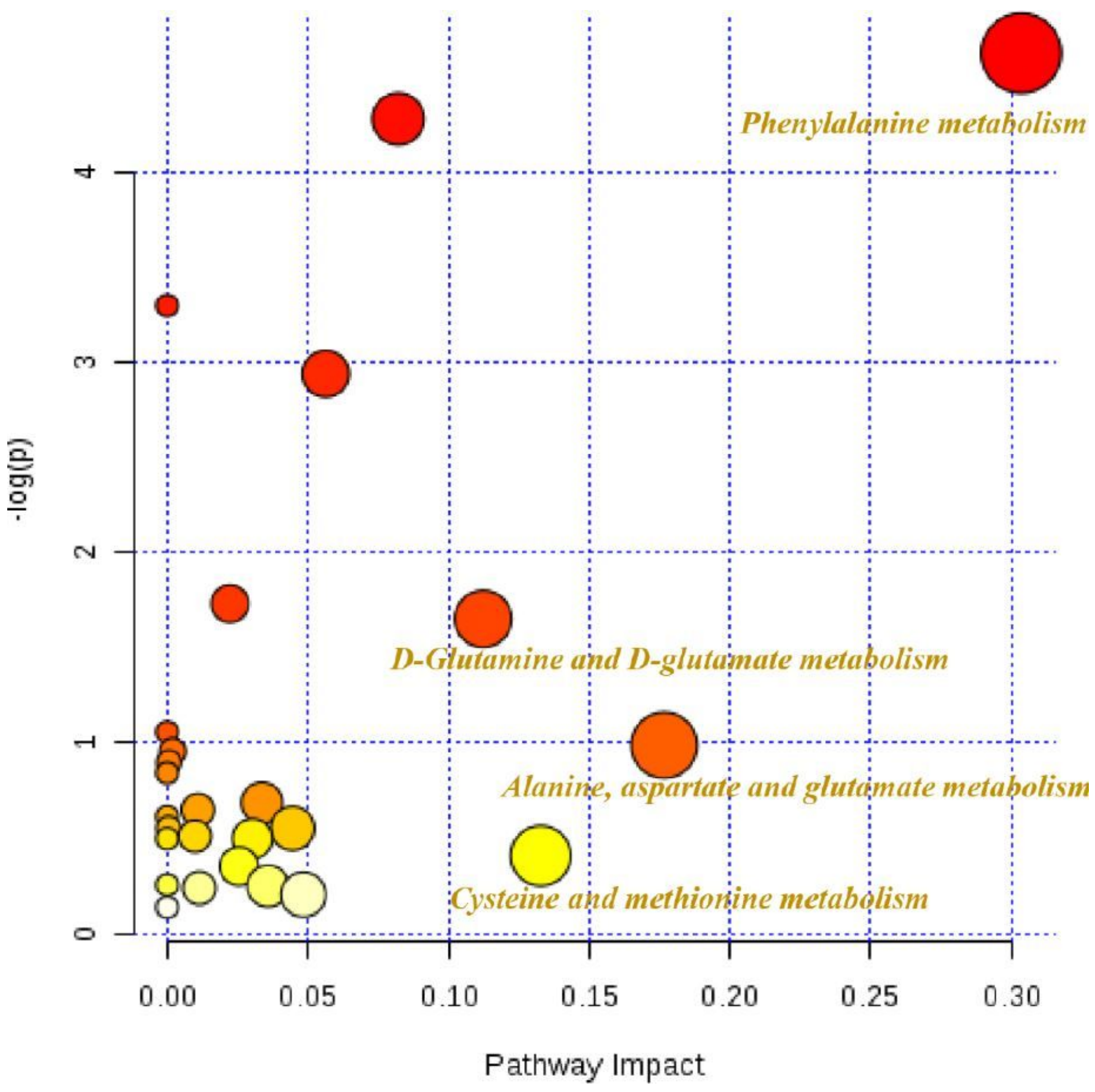

Figure 16

Pathway analysis of biomarkers for $\mathrm{MCl}$ intervention with medium dose of SCPE. 


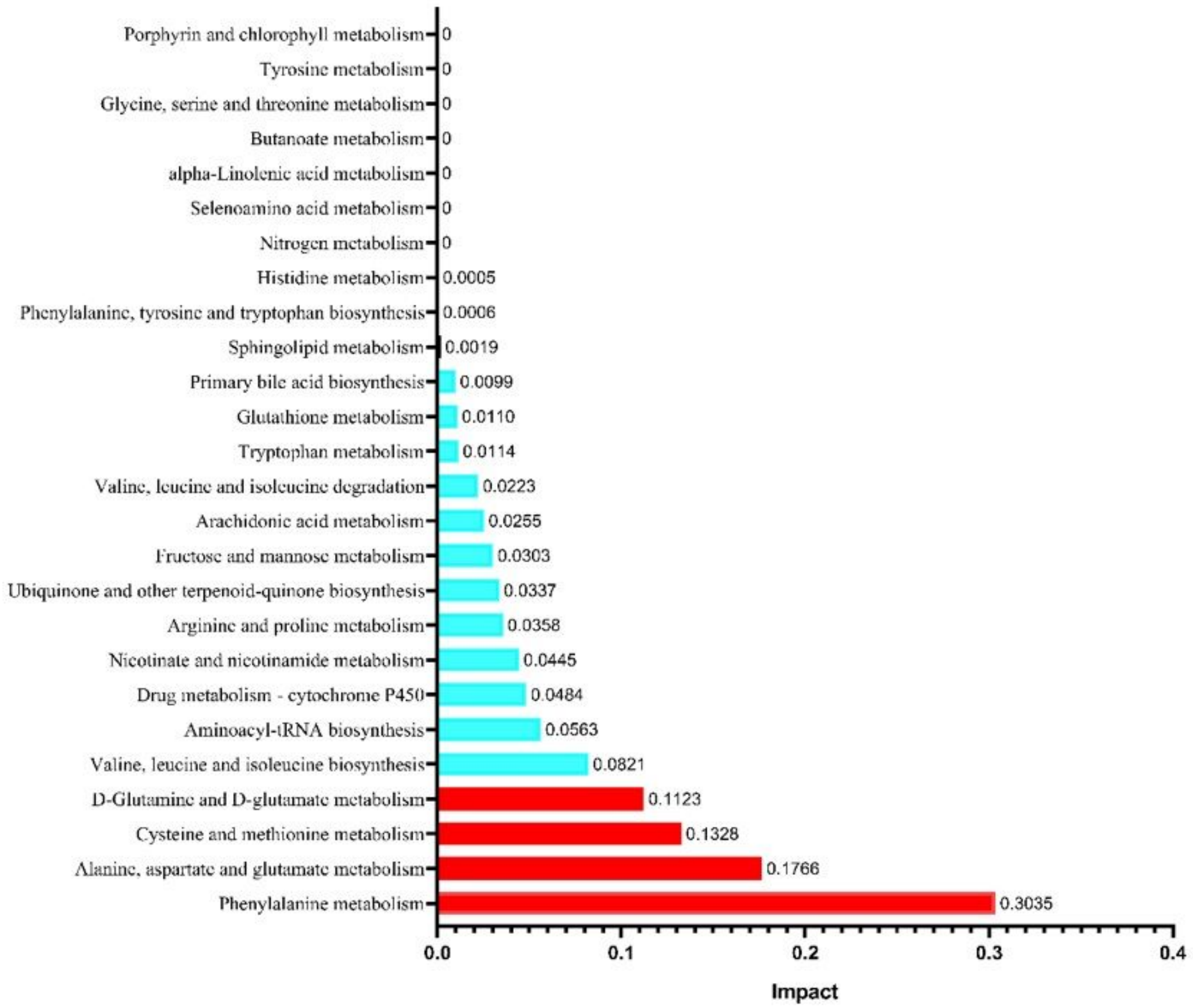

Figure 17

Metabolic pathway enrichment analysis of $\mathrm{MCl}$ biomarkers intervened by SCPE. 


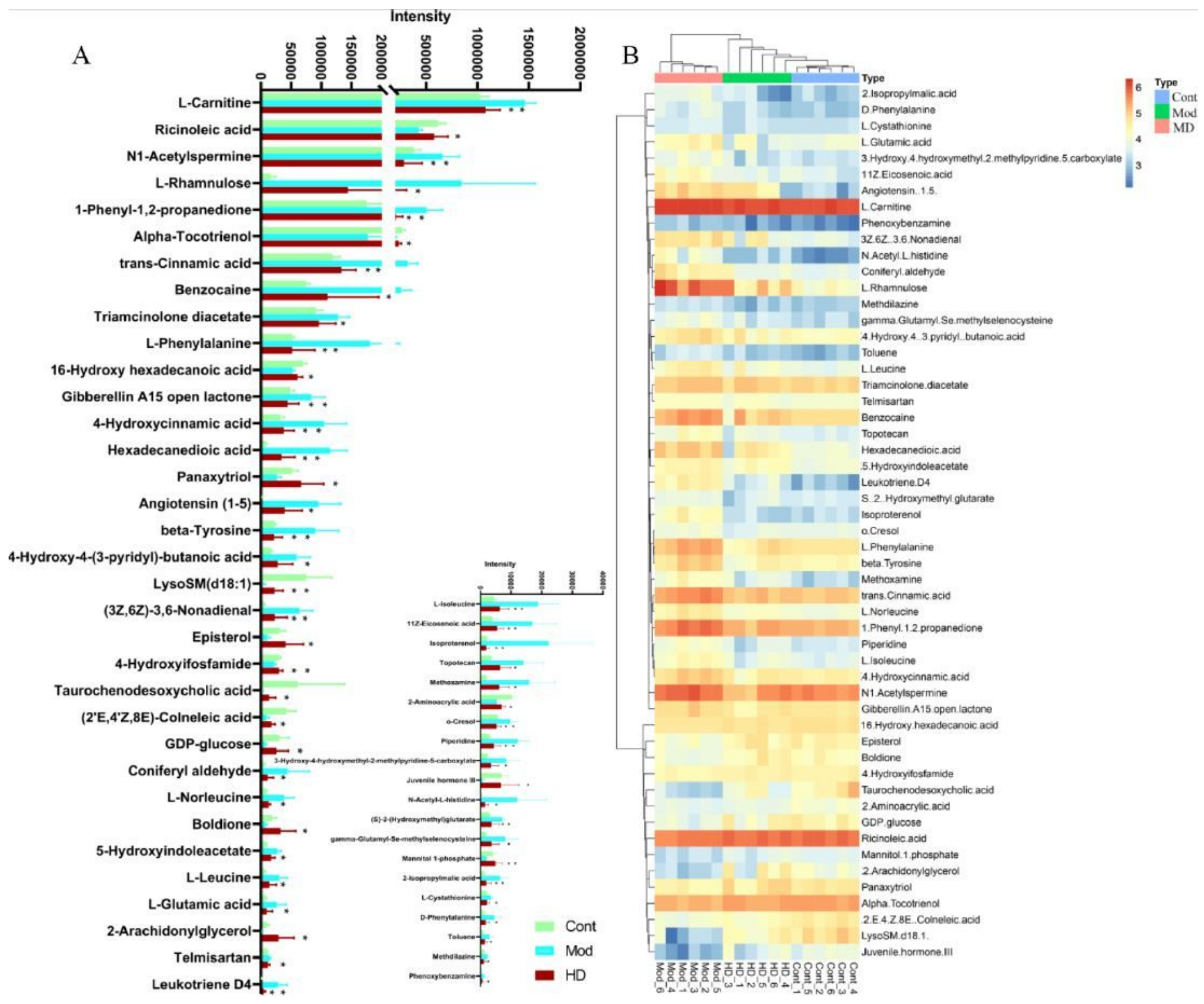

\section{Figure 18}

Metabolic biomarkers of $\mathrm{MCl}$ intervented by high dose of SCPE. (A) Bar chart of metabolic intensity of biomarkers of $\mathrm{MCl}$ treated with high dose (HD), compared with the model group (Mod), *P-value $<0.05$; $\star \star$ P-value $<0.01$; (B) Heat map of biomarkers (red: up-regulated; blue: down-regulated). 


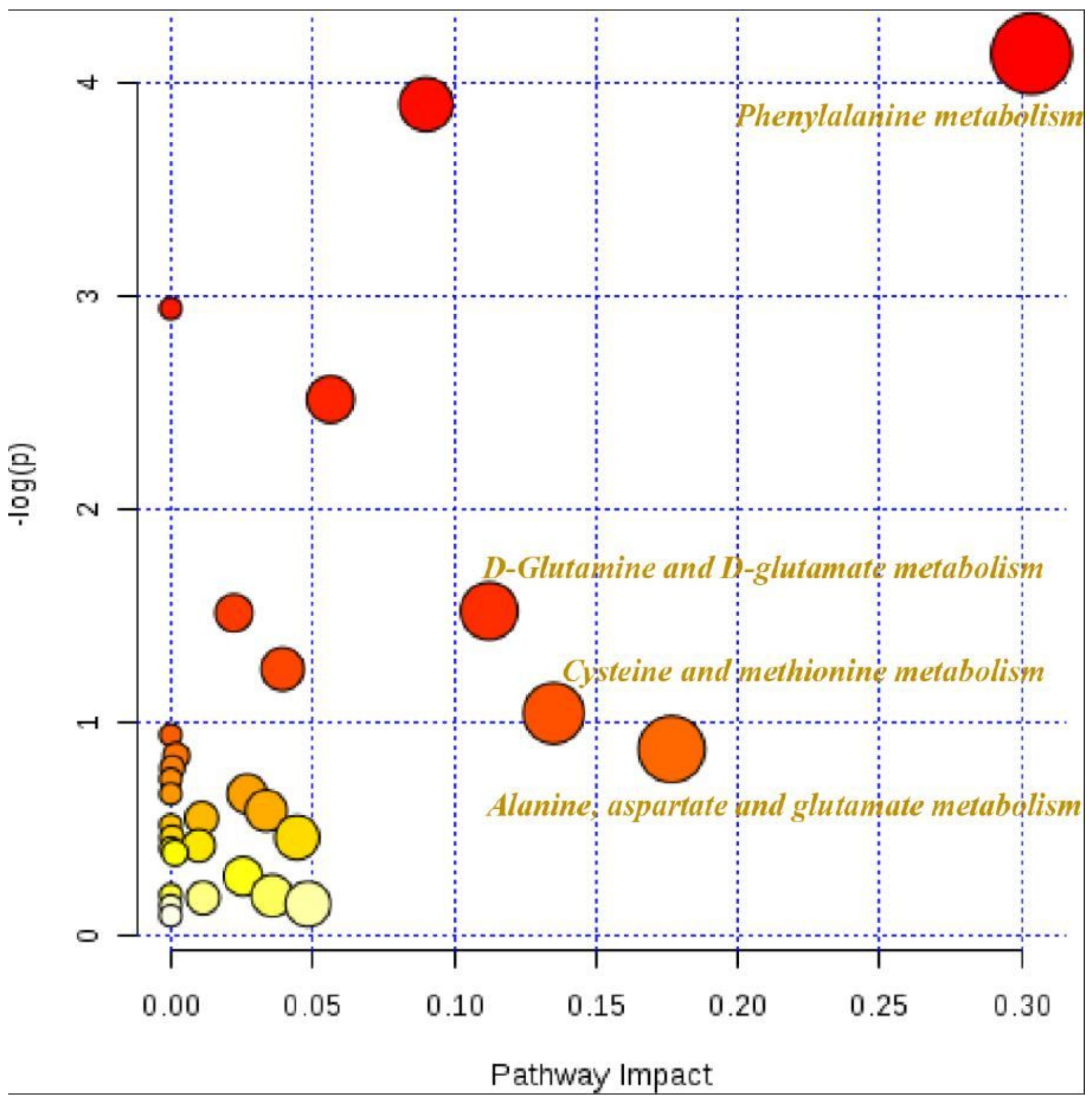

Figure 19

Pathway analysis of biomarkers for $\mathrm{MCl}$ intervention with high dose of SCPE. 
Porphyrin and chlorophyll metabolism -10

Amino sugar and nucleotide sugar metabolism- 0

Tyrosine metabolism- 0

Glycine, serine and threonine metabolism- 0

Butanoate metabolism- 0

Pyruvate metabolism- 0

alpha-Linolenic acid metabolism- 0

Selenoamino acid metabolism- 0

Nitrogen metabolism- 0

Histidine metabolism- 0.0005

Phenylalanine, tyrosine and tryptophan biosynthesis -0.0006 Starch and sucrose metabolism- 0.0015

Sphingolipid metabolism- 0.0019

Primary bile acid biosynthesis- 0.0099

Glutathione metabolism- 0.0110

Tryptophan metabolism- 0.0114

Valine, leucine and isoleucine degradation- $\quad 0.0223$

Arachidonic acid metabolism- $\quad 0.0255$

Vitamin B6 metabolism- $\quad 0.0270$

Ubiquinone and other terpenoid-quinone biosynthesis- $\quad 0.0337$

Arginine and proline metabolism- 0.0358

Fructose and mannose metabolism- 0.0394

Nicotinate and nicotinamide metabolism- $\quad 0.0445$

Drug metabolism - cytochrome P450- 0.0484

Aminoacyl-tRNA biosynthesis- 0.0563

Valine, leucine and isoleucine biosynthesis- 0.0901

D-Glutamine and D-glutamate metabolism- 0.1123

Cysteine and methionine metabolism- 0.1351

Alanine, aspartate and glutamate metabolism-

Phenylalanine metabolism

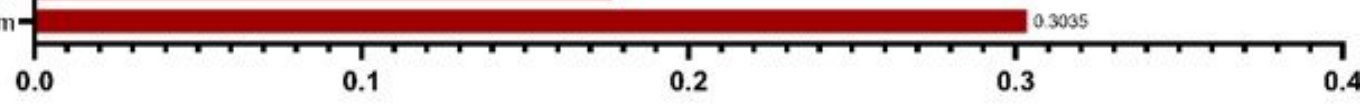

Impact

Figure 20

Metabolic pathway enrichment analysis of $\mathrm{MCl}$ biomarkers intervened by SCPE.

A.

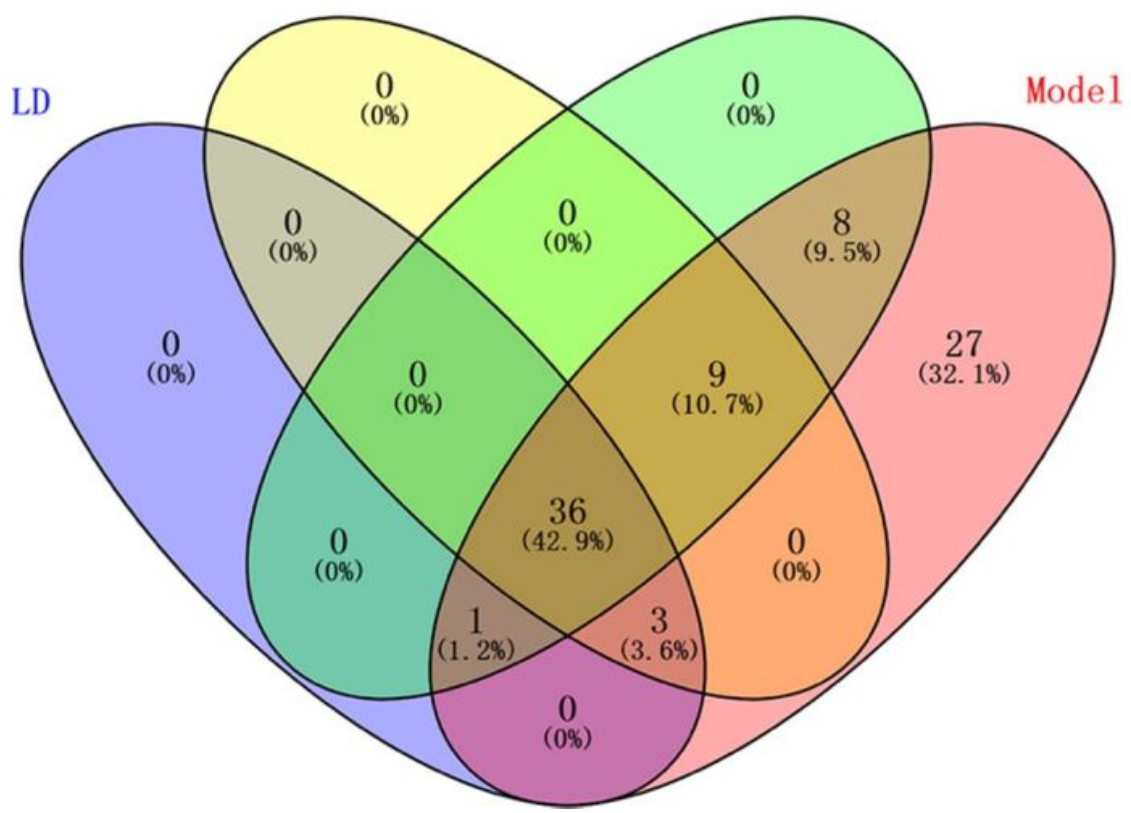

B.

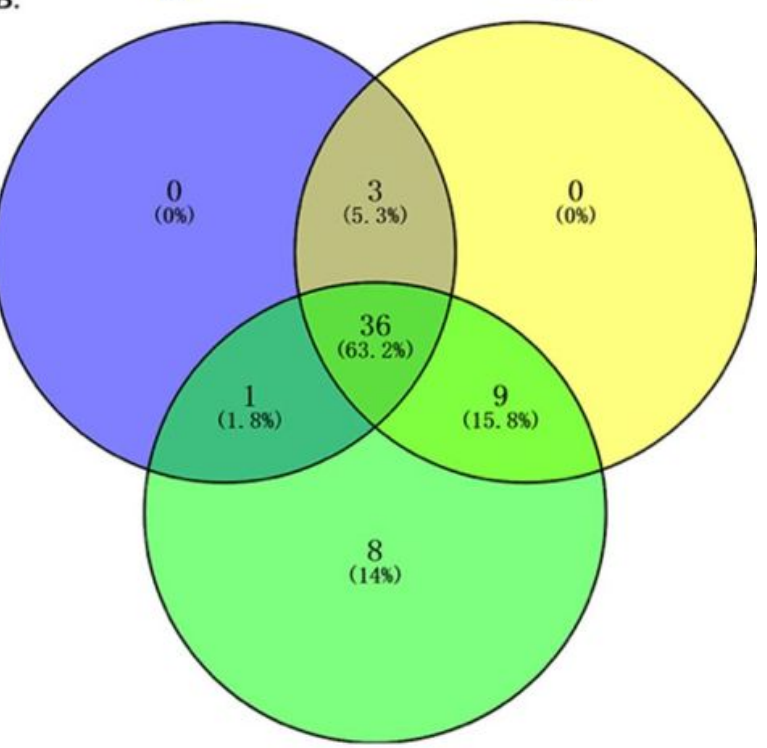

HD

\section{Figure 21}


Venn diagram of metabolic markers in di囚erent doses of SCPE. (A) Model group (Model), Low dose group (LD), Medium dose group (MD), High dose group (HD) Venn diagram; (B) Low dose group (LD), Medium dose group (MD), High dose group (HD) Venn diagram.
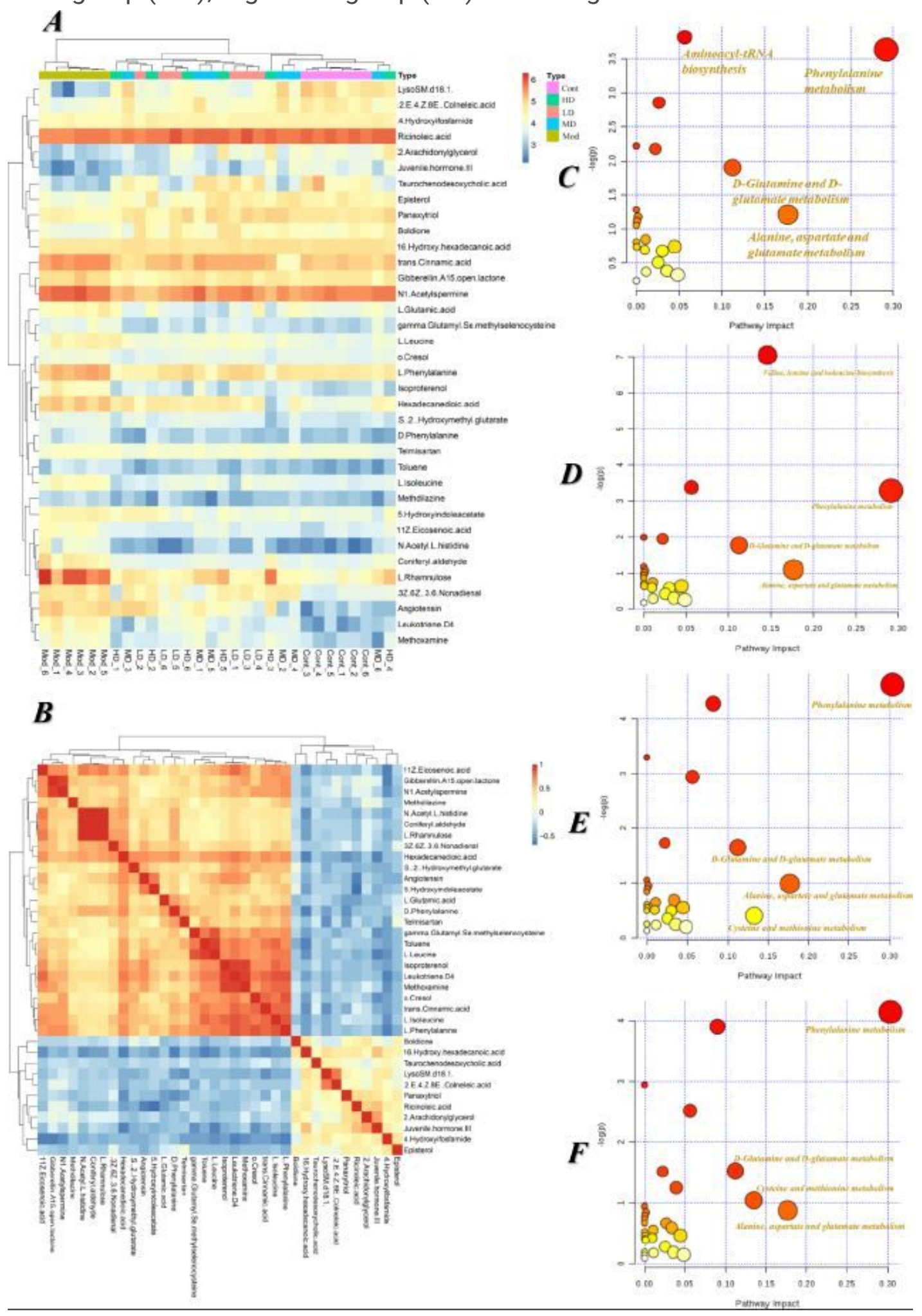

Figure 22

Combination diagram of metabolites analysis. (A) Thermograms of 36 metabolic markers in common callback of low, medium and high dose SCPE; (B) Pearson correlation analysis of 36 metabolic markers 
in the common callback of low, middle and high dose SCPE; (C) The pathway map of 36 metabolic markers for the common callback of low, medium and high dose SCPE; (D) Pathway map of 40 metabolic markers in low dose SCPE callback; (E) The pathway map of 48 metabolic markers for medium dose SCPE callback; (F) The pathway map of 54 metabolic markers for high dose SCPE callback.
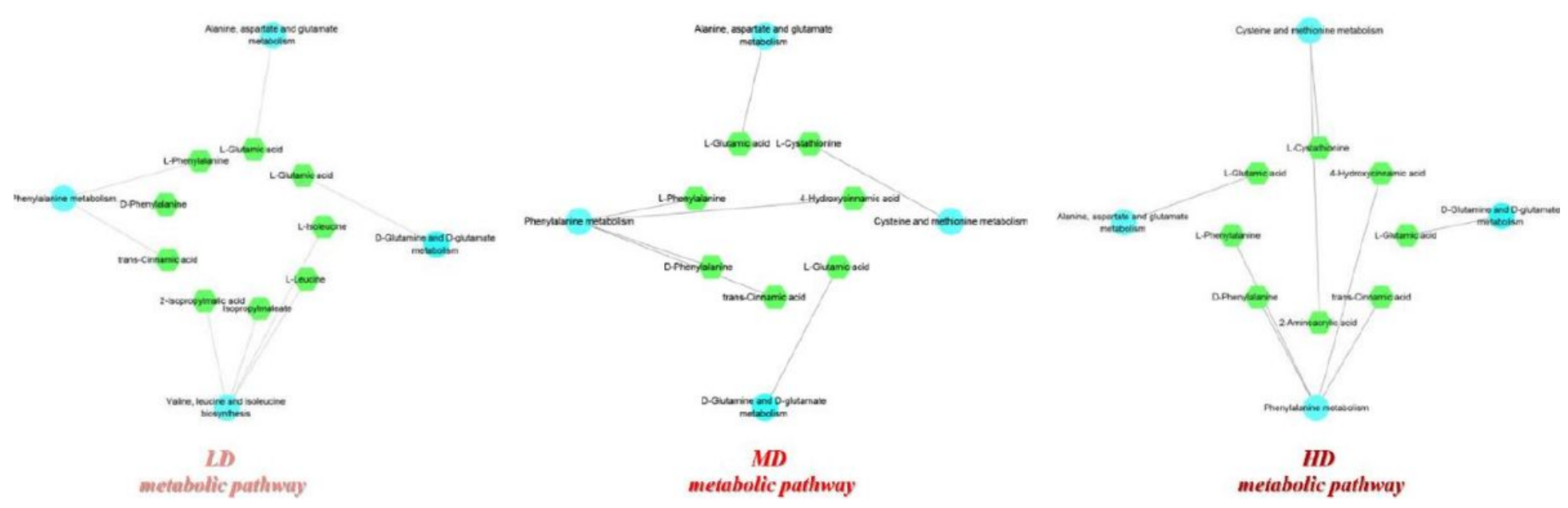

Figure 23

Metabolic pathway analysis.

\section{Supplementary Files}

This is a list of supplementary files associated with this preprint. Click to download.

- supplement26.pdf 